\title{
Turbulence Transport with Nonlocal
}

\section{Interactions}

\section{RECEIVED APR $\cap 61998$ \\ OSTI}

\section{5}

\section{THC QUAITIY INSPECTED :-}

\section{Los Alamos


UC-700 and UC-706

Issued: March 1998

Turbulence Transport with Nonlocal

Interactions

Rodman R. Linn

Timothy T. Clark

Francis H. Harlow

Leaf Turner

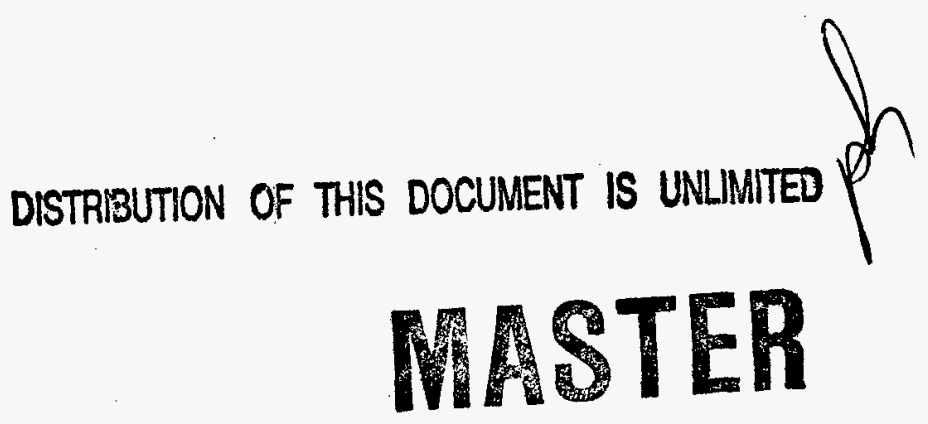




\title{
TURBULENCE TRANSPORT WITH NONLOCAL INTERACTIONS
}

by

\author{
Rodman R. Linn, Timothy T. Clark, Francis H. Harlow, and Leaf Turner
}

ABSTRACT

This preliminary report describes a variety of issues in turbulence transport analysis with particular emphasis on closure procedures that are nonlocal in wave-number and/or physical space. Anomalous behavior of the transport equations for large-scale parts of the turbulence spectrum are resolved by including the physical-space nonlocal interactions. Direct and reverse cascade processes in wavenumber space are given a much richer potential for realistic description by the nonlocal formulations. The discussion also describes issues, many still not resolved, regarding new classes of self-similar form functions.

\section{INTRODUCTION}

\section{A. Scope of the Study}

Turbulence occurs in many circumstances of fluid flow, being driven or sustained by the conversion of large-scale mean-flow energy to intermediate-scale fluctuational energy and dissipated by the entropy-increasing process of cascade to small scales, ultimately to the molecular level where it is manifested in the form of heat.

Our principal goal is to describe nonlocal contributions to closure modeling in both physical and wave-number space and to show that nonlocality of processes lies at the heart of large-structure behavior in nonhomogeneous circumstances.

A second goal is to record some preliminary ideas regarding the existence and usefulness of generic form functions and in particular to show the richness of possibilities for a wide variety of turbulent flows with nonhomogeneous, nonisotropic drive. These form functions may be exact or approximate, depending on circumstances to be described below. They are usually revealed in greatest clarity under circumstances that are described by self-similar combinations of the physical and spectral variables; but they may also occur to a significant level of approximation in localized regions that are continually approaching self-similarity despite the shifting nature of the mean-flow drive.

The current work is focused on far subsonic flows with constant fluid density; collateral activities by our associates show that many of our results can be extended to flows with large variations in material density (e.g., two-phase material interpenetrations) or with large variations in stress-strain behavior. The starting point for analysis is the set of NavierStokes equations, for which we postulate complete relevance in our investigations. With 
constant density, the dependent variables are pressure and velocity. These are assumed to be separable into mean and fluctuating parts (the Reynolds decomposition), described in this report by an overbar and a prime, respectively. Insertion of these symbols into the Navier-Stokes equations and averaging over an appropriate ensemble of realizations lead to the well-known infinite set of transport equations for correlations of all orders. In this process we exploit the complete arbitrariness of choice as to which part of the dynamics is mean flow and which part is turbulence; indeed, we derive much useful guidance for closure modeling by shifting our viewpoint in this regard for several considerations to be described below.

For most of the developments described in this report, we focus on fluid flows that are dominated by mean-flow shear, usually confined to a localized planar shear layer. Some discussion will also be presented on flows in which the free shear is only locally planar.

With the establishment of global or local self-similar forms that reflect the nonisotropy of drive, we then use these forms to take moments of the spectral equations. Besnard, Harlow, Rauenzahn, and Zemach (1992, 1996) (the BHRZ approach) followed this procedure using the spectral form for homogeneous, isotropic turbulent decay and were thereby able to derive single-point turbulence transport equations for the Reynolds stress tensor and for the flux of that tensor through the inertial range of the spectrum. It thus became clear that the "dissipation" tensor in single-point turbulence transport theory actually represents the loss rate of the significant parts of the Reynolds stress components in spectral space, rather than the loss rate that results from molecular viscosity. (These two loss rates become equal only in special circumstances of equilibrium.)

\section{B. Closure Modeling}

The unmodeled turbulence transport equations are exact consequences of the NavierStokes equations. They form an infinite set that is intractable for problem solving; indeed, there is an unresolved question as to whether the set is in principle convergent. On both accounts, it is necessary to find ways to close the set, i.e., to express the $N+1^{\text {st }}$-order correlations in terms of those of order $N$ and lower. For both single-point and twopoint (spectral) modeling, this challenge has been a central focus for numerous previous investigations. The techniques we use to accomplish closure are constrained by numerous requirements, for example,

- preservation of conservation (mass, momentum, and energy),

- correct dimensionality,

- Galilean invariance,

- proper tensor form (tensor invariance), 
- correct behavior of contracted forms,

- realizability,

- simplicity (as defined below), and

- consistency of source with circumstances.

An example of this last requirement is the expression of closure in Reynolds stress transport that ensures the creation of isotropy, only, when the circumstances are isotropic.

A somewhat more nebulous guide to closure modeling is furnished by the postulate that we call separation of processes, which we describe by example. Consider the triple correlation terms arising from the pressure-velocity correlation terms with Poisson-integral substitution for pressure. At least three processes are believed to be described by these terms:

- cascade in $\mathbf{k}$ space,

- diffusion in physical space, and

- return to isotropy.

The postulate means, in effect, that each physical process is described by a modeling term that relates only to that process. The postulate is extremely powerful and useful in enabling a systematic approach to modeling, and we invoke it on many occasions.

The constraint of "simplicity" means the elimination of various higher spatial derivatives or products of lower derivatives from inclusion in a model that otherwise satisfies the other constraints. Motivated by a desire for tractability in problem solving, we usually can find no more justification for the constraint beyond the empirical observation that surprisingly good results can often be obtained from relatively "simple" closure formulations.

Several closure techniques are the following:

- Based on the assumption of a quasi-normal distribution of fluctuations, fourth-order correlations are often expressed as products of second-order correlations.

- Closures for an $N^{\text {th }}$-order correlation are often accomplished through the derivation of its transport equation, the appending of a dissipation term proportional to the correlation, the neglect of both time variations and advection, and a very simple treatment of the $N+1^{\text {st }}$-order terms. In this way the $N^{\text {th }}$-order correlation emerges as a purely algebraic expression of the lower-order quantities. The famous Boussinesq approximation can be obtained in this fashion, and gradient-flux expressions for a variety of closures have likewise been derived.

- A useful closure guidance comes from the idea that the larger-scale part of the turbulence spectrum can be temporarily viewed as mean flow. Source terms for the small-scale part of the turbulence are then modeled from the "mean-flow" driving 
terms, and the loss from the large-scale parts is equated to the gain to the smallscale part. The next step is to return to the viewpoint of considering the entire structure of scales to be turbulence and thus to use the derived source-sink model as a representation of cascade flux through wave-number space.

Other closure modeling techniques are discussed in this report as the need arises. Some of them, especially those that describe nonlocal interactions in both spectral and physical space, are new and serve as the starting point for many of our investigations.

\section{Realizability}

It is useful to summarize some properties of the spectrally integrated Reynolds stress tensor, $R_{i j}$, that are direct consequences of the structure of its definition. These properties give constraints to modeling; closures that lead to violations of these properties are not viable approximations to be used for realistic problem solving; these violations are called unrealizable. The constraints, while necessary, are by no means sufficient, insofar as the ensurance of precise representation is concerned. Much more powerful constraints will no doubt be discovered, based on concepts of generalized information entropy; these ideas are still under development. (Mathematical objectivity may also furnish useful constraints.)

The properties that we summarize here are derived from the tensor structure

$$
R_{i j}=\overline{u_{i}^{\prime} u_{j}^{\prime}} .
$$

Let $e_{i}$ be an arbitrary unit vector. Then

$$
e_{i} e_{j} R_{i j}=\overline{\left(e_{n} u_{n}^{\prime}\right)^{2}} \geq 0
$$

With numerous possible choices of $e_{j}$, one can derive, for example,

$$
\begin{gathered}
2 R_{12} \geq-\left(R_{11}+R_{22}\right) \\
R_{11} \geq 0, R_{22} \geq 0, R_{33} \geq 0 \\
\operatorname{det} R_{i j} \geq 0,
\end{gathered}
$$

and any subdeterminant is likewise positive or zero.

\section{Nonlocality}

Purely local interactions are often the basis for describing transport influences on the evolution of Reynolds stress. In physical space these mean processes that affect Reynolds stress development occur only at the position under consideration. For an incompressible 
fluid, however, pressure effects are communicated instantaneously from their source to other points throughout the fluid. The formal representation of this process is through the integral solution of a Poisson equation, by which the pressure is described by an integral over all space of the product of two velocity gradients. For turbulent transport of Reynolds stress, a key source comes from the ensemble average of correlations between fluctuations of pressure and velocity. In the derivation, the pressure fluctuations can be precisely described by the integral over all space of both the mean and fluctuating velocity components; multiplication of this formal expression by velocity fluctuations and ensemble averaging leads to terms that couple the Reynolds stress to mean-flow gradients to give a turbulence source from all other parts of the flow at each instant in time. Following the derivation of appropriate expressions for this physically nonlocal process, we shall demonstrate its consequences for low-wave-number (large structure) turbulence in terms of its relation to classic laminar instability problems, for which we resolve a seeming discrepancy between turbulence transport theory and the direct solution of the Navier-Stokes equations.

In spectral space, local interactions refer to those in which activity associated with a particular wave number affects the evolution of Reynolds stress only at that same wave number. In colloquial terms, we often describe such processes by saying that a turbulent "eddy" only affects others of its same size. Previous investigators have long recognized the fallacy of this viewpoint. The Eddy-Damped, Quasi-Normal Markovian (EDQNM) approach, for example, introduces triad interactions by which the turbulence at wave number $\mathbf{k}$ is strongly influenced by the behavior at wave numbers $\mathbf{p}$ and $\mathbf{q}$, constrained only by the requirement that $\mathbf{p}+\mathbf{q}+\mathbf{k}=0$, but otherwise summed over all other localities in wave-number space. Again in colloquial terms, we heuristically visualize

- big eddies that spin up small ones or rotate them so as to alter their state of nonisotropy,

- eddies of one size distorting eddies of another size so as to create those of a third size,

- small eddies sucking energy from large ones in the same way that turbulence sucks energy from a mean-flow shear, and

- eddy instability in a mean-flow shear that results in eddy pairs coalescing to form a distinctly larger eddy (the vortex pairing process).

The formal derivation of terms that represent these and other nonlocal processes can only be given with partial rigor. Some key steps that we use in this report are roughly described as follows:

- Two-point correlation functions like the generalized Reynolds stress $R_{i j}(\mathbf{x}, \mathbf{r}, t)$ are often simplified by, in effect, retaining only the lowest terms in a Maclaurin expansion of the separation distance about $\mathbf{r}=0$. Retention of higher order terms results in 
a variety of processes. Correlations arising out of the advective term, for example, exhibit a mean-flow-induced cascade, i.e., the distortion of turbulent structures that alters their size and thus the nature of the two-point correlations as functions of r. The third-order correlation terms arising from advection represent, in analogous fashion, the stretching effects of large-scale turbulent shear on the distortion of smaller-scale structures, which likewise create a Reynolds stress flux (cascade) in wave-number space. We refer to this process as second-point-induced cascade, for which the resemblance to mean-flow-shear-induced cascade is exploited in deriving its representation. These effects are clearly nonlocal in wave-number space. They also have implications for self-diffusion of turbulence or diffusion of a passive scalar in physical space.

- In a similar fashion we retain higher-order terms in the pressure-velocity correlations, which, through the integral Poisson solution, contain both second- and third-order correlation terms. The former couple to mean-flow gradients and, with retention of higher-order terms in the Maclaurin expansion, lead directly to nonlocal turbulence creation in physical space. The latter lead directly to a generalization of representation for turbulent self-diffusion in physical space, in which the effective "eddy viscosity" receives nonlocal contributions from all wave numbers.

A significant issue not resolved in this report is that of higher-order nonlocal effects that could come from the use of finite-domain Poisson-solver integrals for configurations that are only locally homogeneous.

\section{E. Molecular Viscosity}

In most of this report we neglect the effects of molecular viscosity, whose presence is assumed to be manifested only by the conversion to heat of the turbulent energy that has cascaded to very high wave numbers. In single-point turbulence transport equations, the effects of viscosity are awkward to include, whereas in the two-point and spectral representations the effects can be included easily with no approximations. Thus, it is intriguing to ask if turbulence transport theory can be used successfully for solving interesting problems at low Reynolds numbers. In particular, can we investigate the competing processes that convert mean-flow kinetic or potential energy either directly to heat (molecular-scale fluctuations) or to the intermediate state of turbulence? The existence of a critical Reynolds number for transition to turbulence and the dependence of that critical Reynolds number on the level of perturbation are issues that turbulence transport theory should, at least in principle, be capable of addressing. To realize this capability, however, the equations require another feature that most formulations lack, 
namely the ability to describe so-called laminar instability processes. We show in this report that this latter capability requires the inclusion of nonlocal processes in physical space. Potential flow theories for Kelvin-Helmholtz or Rayleigh-Taylor instabilities make crucial use of that nonlocality in the incorporation of inertial effects of fluid lying outside the immediate vicinity of the unstable interface. Turbulence transport theories that lack this capability accordingly create too much turbulence near the interface and too little away from the interface. For turbulence scales smaller than or comparable to the width of the perturbed region, $W$, there is little significance that is lost from the formulations as a result of this deficiency. For larger scales $(|\mathbf{k}| W$ small) the turbulence transport error becomes very large, with significant consequences in regard to the small-wave-number effects on such features as self-similarity of the entire spectral evolution.

With the inclusion of both the molecular viscosity and the nonlocality in physical space, turbulence transport theory should be able to address the features of critical Reynolds number and low-wave-number behavior, although much work remains to be done in this regard.

\section{F. Summary of Basic Equations}

Derivations of various forms for the spectral equations have been given by numerous authors. Our work is based especially on the forms presented by BHRZ, Clark (1992) and Clark and Zemach (1995).

For a fluid with constant density, the starting point is the Navier-Stokes equations for a fluid with constant kinematic viscosity,

$$
\begin{gathered}
\frac{\partial u_{i}}{\partial x_{i}}=0 \\
\frac{\partial u_{i}}{\partial t}+\frac{\partial u_{i} u_{j}}{\partial x_{j}}=-\frac{\partial p}{\partial x_{i}}+\nu \frac{\partial^{2} u_{i}}{\partial x_{j}^{2}}
\end{gathered}
$$

With the standard Reynolds decomposition of flow variables into mean and fluctuating parts,

$$
\begin{gathered}
u_{i}=\bar{u}_{i}+u_{i}^{\prime} \\
p=\bar{p}+p^{\prime}
\end{gathered}
$$

it follows that

$$
\frac{\partial \bar{u}_{i}}{\partial x_{i}}=0 ; \quad \frac{\partial u_{i}^{\prime}}{\partial x_{i}}=0
$$

and

$$
\frac{\partial \bar{u}_{i}}{\partial t}+\frac{\partial \bar{u}_{i} \bar{u}_{j}}{\partial x_{j}}=-\frac{\partial \bar{p}}{\partial x_{i}}+\nu \frac{\partial^{2} \bar{u}_{i}}{\partial x_{j}^{2}}-\frac{\partial R_{i j}}{\partial x_{j}}
$$


in which the Reynolds stress is

$$
R_{i j}=\overline{u_{i}^{\prime} u_{j}^{\prime}} .
$$

In this single-point form for the Reynolds stress, $u_{i}^{\prime}$ and $u_{j}^{\prime}$ are both at the same point in physical space (and also at the same time). From the Navier-Stokes equations it is possible to derive a transport equation for this single-point Reynolds stress:

$$
\begin{aligned}
\frac{\partial R_{i j}}{\partial t} & +\bar{u}_{k} \frac{\partial R_{i j}}{\partial x_{k}}+R_{i k} \frac{\partial \bar{u}_{j}}{\partial x_{k}}+R_{j k} \frac{\partial \bar{u}_{i}}{\partial x_{k}} \\
& +\frac{\partial}{\partial x_{k}}\left(\overline{u_{i}^{\prime} u_{j}^{\prime} u_{k}^{\prime}}\right)=-\frac{1}{\rho}\left(\frac{\partial \overline{u_{i}^{\prime} p^{\prime}}}{\partial x_{j}}+\frac{\partial \overline{u_{j}^{\prime} p^{\prime}}}{\partial x_{i}}\right) \\
& +\frac{1}{\rho} \overline{p^{\prime}}\left(\frac{\partial u_{i}^{\prime}}{\partial x_{j}}+\frac{\partial u_{j}^{\prime}}{\partial x_{i}}\right) \\
& +\nu\left(\frac{\partial^{2} R_{i j}}{\partial x_{k}^{2}}-4 D_{i j}\right),
\end{aligned}
$$

in which

$$
D_{i j}=\frac{1}{2} \overline{\frac{\partial u_{i}^{\prime}}{\partial x_{k}} \frac{\partial u_{j}^{\prime}}{\partial x_{k}}} .
$$

In this form we can see various difficulties with single-point transport models. First is the introduction of several second-order correlations, which typically have been represented by various types of modeling approximations. Second is the appearance of a thirdorder correlation, $\overline{u_{i}^{\prime} u_{j}^{\prime} u_{k}^{\prime}}$, for which a transport equation could also be derived with the introduction of yet more unknown correlations. Third is the introduction of $D_{i j}$, which has the dimensions of Reynolds stress divided by the square of distance. This last quantity is at the heart of single-point Reynolds stress modeling difficulties. How can we determine an appropriate measure of effective turbulence scale that can serve to characterize the dimension of distance that is introduced by $D_{i j}$ ? Many authors [e.g., Daly and Harlow (1970)] identify the loss rate of $R_{i j}$ with viscous dissipation to heat, a fallacy that compounds the difficulty in determining an appropriate length scale. More recently it has been recognized that the loss rate of $R_{i j}$ is properly associated with a flux through the inertial range of wave-number space that can equal the dissipation rate only in very special circumstances.

For this and various other reasons it has become apparent that effective turbulence transport modeling must come to grips with some measure of the size-scale structure of the fluctuations. Although the single-point Reynolds stress is sufficient to describe the effects of turbulence on the mean flow, the evolution of that Reynolds stress is much more satisfactorily described in terms of its two-point generalization,

$$
R_{i j}\left(\mathbf{x}_{1}, \mathbf{x}_{2}\right) \equiv \overline{u_{i}^{\prime}\left(\mathbf{x}_{i}\right) u_{j}^{\prime}\left(\mathbf{x}_{2}\right)}
$$


for which a transport equation can be derived in the form given by BHRZ:

$$
\begin{gathered}
\frac{\partial R_{i j}\left(\mathbf{x}_{1}, \mathbf{x}_{2}\right)}{\partial t}= \\
A_{i j}\left(\mathbf{x}_{1}, \mathbf{x}_{2}\right)-\left\{B_{i j}\left(\mathbf{x}_{1}, \mathbf{x}_{2}\right)+C_{i j}\left(\mathbf{x}_{1}, \mathbf{x}_{2}\right)+\frac{\partial}{\partial x_{1 i}} P_{j}\left(\mathbf{x}_{1}, \mathbf{x}_{2}\right)+\frac{\partial}{\partial x_{1 n}} T_{i n j}\left(\mathbf{x}_{1}, \mathbf{x}_{2}\right)\right\}^{S},
\end{gathered}
$$

where $S$ means that the enclosed expression is to be symmetrized by adding terms with $i \leftrightarrow j, \mathbf{x}_{1} \leftrightarrow \mathbf{x}_{2}$. The quantities on the right are defined by

$$
\begin{aligned}
A_{i j}\left(\mathbf{x}_{1}, \mathbf{x}_{2}\right) & =\nu\left(\nabla_{1}^{2}+\nabla_{2}^{2}\right) R_{i j}\left(\mathbf{x}_{1}, \mathbf{x}_{2}\right) \\
B_{i j}\left(\mathbf{x}_{1}, \mathbf{x}_{2}\right) & =\frac{\partial}{\partial x_{1 n}} u_{n}\left(\mathbf{x}_{1}\right) R_{i j}\left(\mathbf{x}_{1}, \mathbf{x}_{2}\right) \\
C_{i j}\left(\mathbf{x}_{1}, \mathbf{x}_{2}\right) & =\frac{\partial}{\partial x_{1 n}} u_{i}\left(\mathbf{x}_{1}\right) R_{n j}\left(\mathbf{x}_{1}, \mathbf{x}_{2}\right) \\
P_{j}\left(\mathbf{x}_{1}, \mathbf{x}_{2}\right) & =\left\langle p^{\prime}\left(\mathbf{x}_{1}\right) u_{j}^{\prime}\left(\mathbf{x}_{2}\right)\right\rangle / \rho \\
T_{i n j}\left(\mathbf{x}_{1}, \mathbf{x}_{2}\right) & =\left\langle u_{i}^{\prime}\left(\mathbf{x}_{1}\right) u_{n}^{\prime}\left(\mathbf{x}_{1}\right) u_{j}^{\prime}\left(\mathbf{x}_{2}\right)\right\rangle
\end{aligned}
$$

Using Green's theorem, we write, for an infinite domain,

$$
P_{j}\left(\mathbf{x}_{1}, \mathbf{x}_{2}\right)=\int \frac{d \mathbf{x}^{\prime}}{4 \pi\left|\mathbf{x}_{1}-\mathbf{x}^{\prime}\right|} \frac{\partial}{\partial x_{n}^{\prime}} \frac{\partial}{\partial x_{m}^{\prime}}\left\{2 u_{n}\left(\mathbf{x}^{\prime}\right) R_{m j}\left(\mathbf{x}^{\prime}, \mathbf{x}_{2}\right)+T_{m n j}\left(\mathbf{x}^{\prime}, \mathbf{x}_{2}\right)\right\} .
$$

For some purposes [Oberlack and Peters (1993)], it is convenient to introduce the variables

$$
\mathbf{x}=\frac{1}{2}\left(\mathbf{x}_{1}+\mathbf{x}_{2}\right) ; \quad \mathbf{r}=\mathbf{x}_{1}-\mathbf{x}_{2}
$$

and carry through the "spectral" developments completely in terms of the separation variable, $\mathbf{r}$. For the analysis of nonlocal processes in physical space, this procedure is especially convenient, as described in another part of this report. For a truly spectral approach we introduce the Fourier transformation,

$$
R_{i j}(\mathbf{x}, \mathbf{k})=\int e^{-i \mathbf{k} \cdot \mathbf{r}} R_{i j}(\mathbf{x}+\mathbf{r} / 2, \mathbf{x}-\mathbf{r} / 2) d \mathbf{r} .
$$

We also define

$$
E_{i j}(\mathbf{x}, k)=\frac{1}{2} \int R_{i j}(\mathbf{x}, \mathbf{k}) \frac{k^{2} d \Omega_{k}}{(2 \pi)^{3}}
$$


so that $\int E_{\ell \ell}(\mathbf{x}, k) d k$ is the turbulent kinetic energy per unit mass of the fluid. Note that the single-point Reynolds stress tensor that appears in the mean-flow equation is given by

$$
R_{i j}(\mathbf{x}, \mathbf{x})=2 \int_{0}^{\infty} E_{i j}(\mathbf{x}, k) d k
$$

The two-point Reynolds stress transport equation can also be written in terms of $\mathbf{x}$ and $\mathbf{r}$ and Fourier transformed relative to the latter variable. The result can be expressed as

$$
\frac{\partial}{d t} R_{i j}(\mathbf{x}, \mathbf{k})=A_{i j}(\mathbf{x}, \mathbf{k})-\left\{B_{i j}(\mathbf{x}, \mathbf{k})+C_{i j}(\mathbf{x}, \mathbf{k})+2 D_{i j}(\mathbf{x}, \mathbf{k})+F_{i j}(\mathbf{x}, \mathbf{k})+G_{i j}(\mathbf{x}, \mathbf{k})\right\}^{S},
$$

where $S$ now means the addition of like terms in which $i$ and $j$ are interchanged and $\mathbf{k}$ is replaced by $\mathbf{- k}$. The expressions on the right are abbreviations for

$$
\begin{aligned}
A_{i j}(\mathbf{x k}) & =\nu\left(-2 k^{2}+\frac{1}{2} \nabla_{x}^{2}\right) R_{i j}(\mathbf{x}, \mathbf{k}) \\
B_{i j}(\mathbf{x}, \mathbf{k}) & =\left(\frac{1}{2} \frac{\partial}{\partial x_{n}}+i k_{n}\right) Z \bar{u}_{n}(\mathbf{x}) R_{i j}(\mathbf{x}, \mathbf{k}) \\
C_{i j}(\mathbf{x}, \mathbf{k}) & =\left(\frac{1}{2} \frac{\partial}{\partial x_{n}}+i k_{n}\right) Z \bar{u}_{i}(\mathbf{x}) R_{n j}(\mathbf{x}, \mathbf{k}) \\
D_{i j}(\mathbf{x}, \mathbf{k}) & =\left(\frac{1}{2} \frac{\partial}{\partial x_{i}}+i k_{i}\right) \int \frac{e^{-2 i k_{\ell}}\left(x_{\ell}-x_{\ell}^{\prime}\right)}{\pi\left|\mathbf{x}-\mathbf{x}^{\prime}\right|} d \mathbf{x}^{\prime} \\
& *\left(\frac{1}{2} \frac{\partial}{\partial x_{n}^{\prime}}+i k_{n}\right)\left(\frac{1}{2} \frac{\partial}{\partial x_{m}^{\prime}}+i k_{m}\right) Z \bar{u}_{n}\left(\mathbf{x}^{\prime}\right) R_{m j}\left(\mathbf{x}^{\prime}, \mathbf{k}\right) \\
F_{i j}(\mathbf{x}, \mathbf{k}) & =\left(\frac{1}{2} \frac{\partial}{\partial x_{n}}+i k_{n}\right) T_{i n j}(\mathbf{x}, \mathbf{k}) \\
G_{i j}(\mathbf{x}, \mathbf{k}) & =\left(\frac{1}{2} \frac{\partial}{\partial x_{i}}+i k_{i}\right) \int \frac{e^{-2 i k_{\ell}}\left(x_{\ell}-x_{\ell}^{\prime}\right)}{\pi\left|\mathbf{x}-\mathbf{x}^{\prime}\right|} d \mathbf{x}^{\prime} \\
* & \left(\frac{1}{2} \frac{\partial}{\partial x_{n}^{\prime}}+i k_{n}\right)\left(\frac{1}{2} \frac{\partial}{\partial x_{m}^{\prime}}+i k_{m}\right) T_{m n j}\left(\mathbf{x}^{\prime}, \mathbf{k}\right)
\end{aligned}
$$

in which the $Z$ operator is

$$
Z \equiv \exp \left[\frac{i}{2} \nabla_{x}^{(u)} \cdot \nabla_{k}\right]
$$

with $\nabla_{x}^{(u)}$ operating on $\bar{u}_{i}(\mathbf{x})$, and $\nabla_{k}$ operating on any function of $\mathbf{k}$ to its right. $T_{i n j}(\mathbf{x}, \mathbf{k})$ is the Fourier transform of $T_{i n j}\left(\mathbf{x}+\frac{1}{2} \mathbf{r}, \mathbf{x}-\frac{1}{2} \mathbf{r}\right)$. This equation is still an exact development from the Navier-Stokes equations. It serves as the starting point for a variety of approximate representations, including

- truncation of the series expansion for the $Z$ operator after some number of terms, 
- use of local approximations for parts of the integrands in $D_{i j}(\mathbf{x}, \mathbf{k})$ and $G_{i j}(\mathbf{x}, \mathbf{k})$, and

- insertion of EDQNM approximations for $T_{i n j}(\mathbf{x}, \mathbf{k})$.

With the first two of these, BHRZ obtained a form that contains significant spectral information. Using their numbering of terms for further reference we write

$$
\begin{aligned}
& \frac{\partial}{\partial t} R_{i j}(\mathbf{x}, \mathbf{k})=-2 \nu k^{2} R_{i j}(\mathbf{x}, \mathbf{k})+\frac{1}{2} \nu \nabla^{2} R_{i j}(\mathbf{x}, \mathbf{k})-u_{n}(\mathbf{x}) \frac{\partial}{\partial x_{n}} R_{i j}(\mathbf{x}, \mathbf{k})+\frac{\partial u_{n}}{\partial x_{m}} k_{n} \frac{\partial}{\partial k_{m}} R_{i j}(\mathbf{x}, \mathbf{k}) \\
& -\frac{\partial u_{i}}{\partial x_{m}} R_{m j}(\mathbf{x}, \mathbf{k})-\frac{\partial u_{j}}{\partial x_{m}} R_{i m}(\mathbf{x}, \mathbf{k})+2 \frac{\partial u_{n}}{\partial x_{m}} \frac{k_{n}}{k^{2}}\left[k_{i} R_{m j}(\mathbf{x}, \mathbf{k})+k_{j} R_{i m}(\mathbf{x}, \mathbf{k})\right]
\end{aligned}
$$

$$
\begin{aligned}
& +i \frac{k_{m} k_{n}}{k^{2}}\left[k_{i} T_{m n j}(\mathbf{x}, \mathbf{k})-k_{j} T_{m n i}(\mathbf{x},-\mathbf{k})\right]+\frac{1}{2} \frac{k_{m} k_{n}}{k^{2}}\left[\frac{\partial}{\partial x_{i}} T_{m n j}(\mathbf{x}, \mathbf{k})+\frac{\partial}{\partial x_{j}} T_{m n i}(\mathbf{x},-\mathbf{k})\right] \\
& (4 \mathrm{~b}) \\
& +\frac{k_{m}}{k^{2}}\left\{\frac{\partial}{\partial x_{n}}\left[k_{i} T_{m n j}(\mathbf{x}, \mathbf{k})+k_{j} T_{m n j}(\mathbf{x},-\mathbf{k})\right]-\frac{k_{n} k_{\ell}}{k^{2}} \frac{\partial}{\partial x_{\ell}}\left[k_{i} T_{m n j}(\mathbf{x}, \mathbf{k})+k_{j} T_{m n i}(\mathbf{x},-\mathbf{k})\right]\right\} \\
& (4 \mathrm{c}) \\
& -i k_{n}\left[T_{i n j}(\mathbf{x}, \mathbf{k})-T_{j n i}(\mathbf{x},-\mathbf{k})\right]-\frac{1}{2} \frac{\partial}{\partial x_{n}}\left[T_{i n j}(\mathbf{x}, \mathbf{k})+T_{j n i}(\mathbf{x},-\mathbf{k})\right] .
\end{aligned}
$$

Note that the overbars have been dropped at this stage from the mean-flow velocity.

From this equation, BHRZ derive a transport equation for $E_{i j}(\mathbf{x}, k)$ by taking a suitable integral over angles in $\mathbf{k}$ space. For our initial purposes of examination of nonlocal closure models, an angularly averaged version of the equation has many advantages of simplicity and tractability for numerical testing. There are some significant losses, however, in working with the angularly integrated equation. In particular we lose the important relationship between nonisotropy in $\mathbf{k}$ space and nonisotropy in physical space, which, in some respects, makes the analysis conceptually simpler, although much more difficult in the mechanics of accomplishment. 
It is not a straightforward process to perform the angular integrations in $\mathbf{k}$ space. BHRZ note that quantities like $-2 \nu k^{2} R_{i j}(\mathbf{x}, \mathbf{k})$ are trivially integrable over angles in $\mathbf{k}$ space, while others like

$$
\int \frac{k_{n} k_{i}}{k^{2}} R_{m j}(\mathbf{x}, \mathbf{k}) \frac{k^{2} d \Omega_{k}}{(2 \pi)^{3}}
$$

are not. They postulate a form for the integral based on the available tensors, considerations of dimensionality, and requirements for various contractions. Alternatively, we can look for self-similar forms that are functions of combinations of spectral and spatial variables, parameterized by functions of $\mathbf{x}$ and $t$ only. BHRZ used this form-function approach in taking moments of their $E_{i j}(\mathbf{x}, k)$ equation to arrive at single-point equations, but their form functions are those of purely isotropic homogeneous turbulence decay. Nevertheless, they succeeded in obtaining $R_{i j}-\epsilon_{i j}$ and $k-\epsilon$ single-point models that show more clearly than ever before the origin of loss rate for $R_{i j}$. Our goal is to obtain much more general form functions and to use them directly for moments in both the angular and magnitude integrals within $\mathbf{k}$ space.

With operators

$$
\mathbf{V}=\nu\left(-2 k^{2}+\frac{1}{2} \nabla^{2}\right)
$$

and

$$
\mathbf{T}=\frac{\partial}{\partial x_{n}} \nu_{T} \frac{\partial}{\partial x_{n}}-c_{1} \frac{\partial}{\partial k} k^{2} \sqrt{k E}+c_{2} \frac{\partial}{\partial k} k^{3} \sqrt{k E} \frac{\partial}{\partial k},
$$

the BHRZ transport equation for $E_{i j}(\mathbf{x}, k)$ is

$$
\begin{aligned}
\frac{\partial E_{i j}}{\partial t}+\bar{u}_{n} \frac{\partial E_{i j}}{\partial x_{n}} & =\mathbf{V}\left[E_{i j}\right]+\mathbf{T}\left[E_{i j}\right]-\left(\frac{\partial \bar{u}_{i}}{\partial x_{n}} E_{n j}+\frac{\partial \bar{u}_{j}}{\partial x_{n}} E_{i n}\right) \\
& +c_{B} A_{i j}+c_{B 1} B_{i j}+c_{B 2} C_{i j} \\
& +\frac{\partial}{\partial k} k\left[c_{F} A_{i j}+c_{F} B_{i j}+c_{F 1} C_{i j}+c_{F 2} \delta_{i j} \frac{\partial \bar{u}_{n}}{\partial x_{m}} E_{n m}\right] \\
& +c_{M} k \sqrt{k E}\left(\frac{1}{3} E \delta_{i j}-E_{i j}\right)
\end{aligned}
$$

in which

$$
\begin{aligned}
A_{i j} & =\frac{\partial u_{i}}{\partial x_{n}} E_{n j}+\frac{\partial u_{j}}{\partial x_{n}} E_{i n}-\frac{2}{3} \delta_{i j} \frac{\partial u_{n}}{\partial x_{m}} E_{n m} \\
B_{i j} & =\frac{\partial u_{n}}{\partial x_{i}} E_{n j}+\frac{\partial u_{n}}{\partial x_{j}} E_{i n}-\frac{2}{3} \delta_{i j} \frac{\partial u_{n}}{\partial x_{m}} E_{n m} \\
C_{i j} & =\left(\frac{\partial u_{i}}{\partial x_{j}}+\frac{\partial u_{j}}{\partial x_{i}}\right) E
\end{aligned}
$$


are mean-flow coupling terms that all have vanishing contraction and accordingly give no contribution to the creation of overall turbulence energy, $E \equiv E_{\ell \ell}$. Also

$$
\nu_{t}=C_{D} \int_{0}^{\infty} \sqrt{k E} \frac{d k}{k^{2}}
$$

is a model for the effective turbulence eddy viscosity. The examination of various contraction constraints shows that

$$
\begin{gathered}
c_{B 1}=8 c_{B}-6, \quad c_{B 2}=-3 c_{B}+11 / 5 \\
c_{F}=-3 c_{B} / 2+1, \quad c_{F 1}=c_{B}-7 / 10, \quad c_{F 2}=7 c_{B} / 2-8 / 3
\end{gathered}
$$

while $c_{B}, c_{D}, c_{1}, c_{2}$, and $c_{m}$ are five dimensionless constants yet to be specified. As discussed by Clark (1992), there is reason to believe that the values of these constants should be related to the Kolmogorov constant, $c_{k}$, by

$$
\begin{aligned}
c_{1}+\frac{5}{3} c_{2} & =C_{K}^{-3 / 2}, \\
c_{1} & =\frac{6}{11} C_{K}^{-3 / 2}, \\
c_{2} & =\frac{3}{11} C_{K}^{-3 / 2}, \\
c_{m} & =\frac{26}{33} C_{K}^{-3 / 2},
\end{aligned}
$$

and that

$$
c_{B}=3 / 4
$$

It is believed that $C_{K}$ is approximately 1.5. The value of $c_{D}$ is less well determined; we resolve the matter somewhat by considering the important nonisotropic contributions that can occur in the turbulence self-diffusion near a free shear layer.

The spatial diffusion $\mathbf{T}\left(E_{i j}\right)$ contains a term

$$
\frac{\partial}{\partial x_{n}}\left(\nu_{t} \frac{\partial E_{i j}}{\partial x_{n}}\right) \text {. }
$$

There are both conceptual and practical difficulties with this particular formulation. Spatial diffusion approximates two very different processes. In the triple correlation term arising from advection,

$$
\frac{\partial}{\partial x_{n}}\left(\overline{u_{i}^{\prime} u_{j}^{\prime} u_{n}^{\prime}}\right)
$$

the flux should have complete symmetry among the indices $i, j$, and $n$; in the $\mathbf{T}$ operator this requirement is clearly violated. The pressure-velocity correlation term

$$
\frac{\partial \overline{u_{i}^{\prime} p^{\prime}}}{\partial x_{j}}+\frac{\partial \overline{u_{j}^{\prime} p^{\prime}}}{\partial x_{i}}
$$


depends on spatial variations in the $i$ and $j$ directions; such forms are not present in the expression for $\mathbf{T}$. In addition to these conceptual difficulties, there is a practical one. With the particular diffusion term in $\mathbf{T}$, the calculation of turbulence evolution in a free shear layer exhibits a striking sensitivity to the value of $c_{D}$. For only one particular value can self-similarity be sustained; for any other value the solution degenerates because the turbulence diffuses either too slowly or too rapidly in comparison to the growth rate of the shear-layer width. The matter is discussed further in Chapter V.

BHRZ discuss some motivations for splitting $E_{i j}$ into a diagonal part and a deviator part,

$$
E_{i j}=\frac{1}{3} E \delta_{i j}+\tilde{E}_{i j}
$$

thereby obtaining the transport equations,

$$
\begin{gathered}
\frac{\partial E}{\partial t}+u_{n} \frac{\partial E}{\partial x_{n}}=\mathbf{V}[E]+\mathbf{T}[E]-2 \frac{\partial \bar{u}_{n}}{\partial x_{m}} \tilde{E}_{n m}+3 c_{F 2} \frac{\partial}{\partial k}\left(k \frac{\partial \bar{u}_{n}}{\partial x_{m}} \tilde{E}_{n m}\right) \\
\frac{\partial \tilde{E}_{i j}}{\partial t}+u_{n} \frac{\partial \tilde{E}_{i j}}{\partial x_{n}}=\mathbf{V}\left|\tilde{E}_{i j}\right|+\mathbf{T}\left|\tilde{E}_{i j}\right|+\left(c_{B}-1\right) \tilde{A}_{i j}+c_{B 1} \tilde{B}_{i j}-2 C_{i j} / 15 \\
+\frac{\partial}{\partial k} k\left[c_{F} \tilde{A}_{i j}+c_{F} \tilde{B}_{i j}-C_{i j} / 30\right]-c_{M} k \sqrt{k E} \tilde{E}_{i j}
\end{gathered}
$$

The motivation for this split lies in its ability to describe the very different high-wavenumber behaviors for $E$ and $\tilde{E}_{i j}$, which vary in the self-similar inertial range as $k^{-5 / 3}$ and $k^{-7 / 3}$.

The terms in the transport equation for $E$ exhibit their physical interpretations quite well. The left side of the equation describes changes of $E$ along the mean motion of the fluid flow. $V[E]$ denotes the effects of molecular viscosity, combining the dissipation to heat (principally at high wave numbers, as indicated by the factor $k^{2}$ ) and the diffusion of the turbulent energy. $T[E]$ describes the transport of turbulence in both physical space (turbulence self-diffusion) and wave-number space (cascade, the true basis for loss of turbulence energy from low wave numbers). $T[E]$ is presented in the form of models for the triple correlation terms that occur in both the advection and in the integral solution of the Poisson equation for pressure, which has been used in the pressure-velocity correlation terms. (These models are considerably altered and extended in the investigations described in this report.) The term $-2 \tilde{E}_{n m} \partial \bar{u}_{n} / \partial x_{m}$ represents the source to turbulence energy from gradients in the mean-flow. Integration of this term over all values of $k$ gives the total source of turbulence energy, which is exactly conservative in consideration of the sink to mean-flow energy as described by the evolution equation for $\bar{u}_{i}$ converted to a transport equation for $\frac{1}{2} \bar{u}_{\ell} \bar{u}_{\ell}$. The $c_{F}$ term in the $E$ equation describes a lowest-order approximation 
to the mean-flow-induced cascade of turbulence energy in $k$ space. As written, this term represents the effects of eddy distortion by the mean flow, or in colloquial terms, the stretching of an eddy so as to thin it out to a smaller cross-section size. We describe a significant generalization of this term to include the nonlocal effects of large-eddy shears distorting the structure of small eddies and most particularly to the extreme limit of meanflow free shears inducing the coalescence of smaller eddies (the vortex-pairing process). This last step of generalization requires the sorting out of a very specific process from the enormous number of complex nonlocal interactions represented by both the second- and third-order correlation functions. (It is worth noting that second-order correlations that couple to mean flow give a special case of nonlocal coupling in $k$ space that is accompanied in both the advective and the pressure-velocity processes by third-order correlation terms representing turbulence coupling to larger eddies. Again, this observation is closely related to the concept that large structures can be characterized equally well as part of the turbulence or part of the mean flow, which is a concept we believe can be exploited to great advantage in the accomplishment of modeling.)

The transport equation for $\tilde{E}_{i j}$, the deviatoric part, has a directly analogous interpretation of terms. In addition that equation contains a $c_{m}$ term, which, in reference to the $E_{i j}$ transport equation, describes the tendency for return to isotropy; for the deviatoric part, this process is purely one of decay. As modeled here, the rate of return to isotropy depends only on the strength of the turbulence at the wave number in question; we show that this rate and others (e.g., of cascade) are much more appropriately determined as the sum of effects from all parts of $k$ space. Note that whereas $E$ has only a cascade decay (neglecting molecular viscosity), $\tilde{E}_{i j}$ has two decay processes, cascade and return to isotropy.

This double decay has consequences for modeling the so-called dissipation tensor, $\epsilon_{i j}$, in single-point theories. Some authors [e.g., Daly and Harlow (1970) and Linn (1997)] have suggested that $\epsilon_{i j}$ be proportional to $R_{i j}$ while others claim that $\epsilon_{i j}$ should have only diagonal components. Here we can see that the issue is related to the comparison between cascade flux rate (for the decay of $E$ ) and return-to-isotropy rate (for the decay of $\tilde{E}_{i j}$ ). As discussed in this report, both of these rates have nonlocal generalizations from which we can draw conclusions related to the modeling of $\epsilon_{i j}$.

The next step in the evolution of equations is the determination of self-similar form functions to be used in performing moment integrals in $k$ space and thus arriving at singlepoint models for practical calculations in science and engineering applications. BHRZ show an excellent example of this procedure based on the self-similar forms for homogeneous, isotropic turbulent decay. Their result is a particular form for the well-known heuristically 
derived equations generally known as $R_{i j}-\epsilon_{i j}$ (and $k-\epsilon$ ) models, which are not described in this introductory summary. With more general self-similar form functions, we derive moments that produce extensions to the $R_{i j}-\epsilon_{i j}$ models for use with mean-flow drives that are strongly nonhomogeneous and nonisotropic. Comparisons among models are presented in another part of this report.

\section{G. The Boussinesq Approximation}

To estimate the tensor structure of the Reynolds stress, it is often convenient to use an approximate representation that bears a close resemblance to the Stokes tensor for molecular dynamics. In its simplest realization, this representation is constructed of "available" tensors, constrained by dimensionality and correctness of contractions. For the spectrally integrated Reynolds stress, $R_{i j}$, the approximation is usually given in the form

$$
R_{i j}(\vec{x}, t)=\frac{1}{3} R_{n n} \delta_{i j}-\nu_{t}\left(\frac{\partial \bar{u}_{i}}{\partial x_{j}}+\frac{\partial \bar{u}_{j}}{\partial x_{i}}\right)
$$

A heuristic derivation of this expression can be based on the full Reynolds stress transport equation, closed by modeling and spectrally integrated. That equation describes the various sources and sinks to time variations of $R_{i j}$; during circumstances of rapid changes in drive, the terms contribute in varying proportions to $\partial R_{i j} / \partial t$. As the drive settles to steady or self-similar form, the contributing terms tend more to balance each other than to feed the Reynolds stress growth. It is this balance (principally among shear creation, return to isotropy, and decay) that leads to the Boussinesq approximation.

Similar balances for variable-density turbulence lead to analogous approximations, Steinkamp (1996) and Steinkamp, Clark, and Harlow (1995).

These approximations can be extended to include some of the effects of time variations. The starting point is the $R_{i j}$ transport equation in the following form:

$$
\begin{aligned}
\frac{d R_{i j}}{d t} & =\frac{\omega \sqrt{K}}{35} R_{n n} \delta_{i j}-\frac{\omega \sqrt{K}}{s} R_{i j} \\
& -R_{i k} \frac{\partial \bar{u}_{j}}{\partial x_{k}}-R_{j k} \frac{\partial \bar{u}_{i}}{\partial x_{k}}
\end{aligned}
$$

in which $\omega$ is a constant, $K=R_{n n} / 2$, and $d / d t$ is the time derivative along the mean flow. Thus

$$
\begin{aligned}
R_{i j} & =\frac{1}{3} R_{n n} \delta_{i j}-\frac{s}{\omega \sqrt{K}} \frac{d R_{i j}}{d t} \\
& -\frac{s}{\omega \sqrt{K}}\left(R_{i n} \frac{\partial \bar{u}_{j}}{\partial x_{k}}+R_{j k} \frac{\partial \bar{u}_{i}}{\partial x_{k}}\right) .
\end{aligned}
$$


Substitute this equation into itself; the result is, to lowest order,

$$
R_{i j}=\left(\frac{2}{3} K-\frac{2 s}{3 \omega \sqrt{K}} \frac{d K}{d t}\right) \delta_{i j}-\frac{2}{3 \omega} s \sqrt{K}\left(\frac{\partial \bar{u}_{i}}{\partial x_{j}}+\frac{\partial \bar{u}_{j}}{\partial x_{i}}\right)
$$

Numerous previous studies have identified

$$
\frac{2 s \sqrt{K}}{3 \omega} \equiv \nu_{t}
$$

with $\omega$ determined by

$$
\frac{2}{3 \omega} \approx 0.09
$$

or, $\omega \approx 7.4$. Thus, the usual Boussinesq approximation is extended to approximate transient effects through modification of the diagonal part of the Reynolds stress tensor. Note that realizability is violated if the coefficient of $\delta_{i j}$ is negative. Indeed, this approximation requires

$$
\frac{d K}{d t} \ll \frac{7.4 K^{\frac{3}{2}}}{s}
$$

for validity. 


\section{NONLOCAL PROCESSES IN WAVE-NUMBER SPACE}

\section{A. Introductory Description}

Turbulent structures at specific length scales do not have a one-to-one correspondence with the structures at specific wave numbers. For heuristic conceptualization we nevertheless associate with profit the large-scale processes with small wave numbers and vice versa.

Mean-flow free shear is an interesting feature that combines both large and small wave numbers, looking like a very large sharp-edged vortex, i.e., a transition from solid-body rotational flow inside to a fluid at rest outside. The sharp-edged transition is rich in high wave numbers; the large size gives some distinctive low wave number components. Thus the classic mean-flow source terms in the turbulence transport equations represent a special case of nonlocal interaction in $\mathbf{k}$ space, in which both very small $(|\mathbf{k}| \rightarrow 0)$ and very large wave numbers interact with turbulence at a specific wave-number to enhance its energy.

The actual large structures of what we would ordinarily identify as turbulence do not necessarily have the wave number properties of a free shear layer but, nevertheless, can interact with the turbulence at any specific wave number to transfer Reynolds stress strength to that wave-number, as described by triple-correlation terms from both the advection and pressure-velocity parts of the transport equation. Indeed, for each of the mean-flow coupling terms there is a corresponding triple-correlation term, which describes more generally the coupling from all other scales. The part that behaves like a mean-flow shear source to the turbulence can be modeled in a form that we call second-point direct cascade. It is only one of the nonlocal processes that we have modeled and tested in our investigations. Altogether there are six types that we have considered.

1. Mean-flow-induced direct cascade, i.e., eddy distortion in mean-flow shear.

2. Second-point direct cascade (eddy distortion and feeding from large-scale structures and depletion by feeding to small-scale structures).

3. Mean-flow-induced reverse cascade (vortex pairing).

4. Second-point-induced reverse cascade (vortex pairing in large-structure shear layers; also the part of the vortex distortion leading to smaller $k$ ).

5. Spontaneous reverse cascade (attraction of vortex pairs during parallel alignmentespecially prominent in turbulence constrained to two dimensions).

6. Nonlocal rates for local cascade models, local return-to-isotropy descriptions, and similar processes in which the rate contributions from all wave numbers determine the evolution at any particular wave number. 
Note that a special case of number 2 is the cascade associated with the idealized concept of the instability of momentarily aligned vortices even at the same scale, in which one of the pair is captured somewhere along its line by the other and pulled around in "hairpin" fashion. Note also that the mean-flow-induced reverse cascade is not a conservative process in wave-number space.

The existence of a local (same-wave-number) contribution to each of these processes does not, however, justify its local representation in turbulence spectral transport equations. The success of local representations results from an entirely different concept, namely the strong tendency for the structure of turbulence to continuously approach selfsimilarity ("universality" of form), which enables the properties of the entire spectrum to be determined with surprising accuracy from the purely local properties of the spectrum. Chapter IV discusses these matters in more detail.

The formal source to each of these nonlocal processes lies in higher-order terms in Maclaurin expansions about $\mathbf{r}=0$ and in the use of a completely nonlocal integrand in the double- and triple-correlation parts of the Green's function solution for the pressurevelocity correlation terms. The EDQNM approach shows that triple-correlation terms can be approximated by integrals of triad interactions over all of wave-number space: structures at wave number $\mathbf{p}$ interact with those at wave number $\mathbf{q}$ to produce changes at wave number $\mathbf{k}$, with the constraint that $\mathbf{p}+\mathbf{q}+\mathbf{k}=0$. For circumstances with significant nonhomogeneity and nonisotropy, however, the usual techniques of EDQNM are of questionable validity, although they have had a strong influence in guiding many parts of our investigations.

Thus our procedure has been to postulate the forms for nonlocal models with due recognition of the numerous constraints that must be observed, as described in the Introduction. As we shall show, there is remarkably little freedom in the choice of models. Single-point $k-\epsilon$ and related models succeed as well as they do because of the stringent nature of these constraints. At the single-point $k-\epsilon$ level of complexity, the dominant constraints are those of dimensionality and Galilean invariance, together with the more nebulous idea of involving only low-order derivatives and no products of derivatives. At the single-point $R_{i j}-\epsilon_{i j}$ level, the constraint of proper tensor forms is added, together with the requirements of physical "cause and effect," e.g., isotropic drive circumstances cannot produce nonisotropic turbulence. (But note a discussion by Clark (1992) that seems to contradict this constraint, in which "hidden" spectral components of nonisotropy can serve as a source for overall integral nonisotropy.) Progressively greater complexity (i.e., spectral models in $k$ space or $\mathbf{k}$ space) would seem to increase the freedom of choice for the terms, but at each stage there are additional constraints that severely curtail that freedom. Thus 
there is much optimism for the discovery of viable models through the route of constrained postulates. Also there is much incentive, in that the achievement of rigorous derivations is likely a long way off; and even the approximate derivations like those of EDQNM have highly restricted applicability.

\section{B. Mean-Flow-Induced Direct Cascade and Reverse Cascade (Items 1 and 3)}

In the spectral derivation of a transport equation for $R_{i j}(\mathbf{x}, \mathbf{k}, t)$, we note in the list in Section II.A that the term (2b)-which is in the Reynolds stress transport equation in Section I.F-describes a conservative flux in $\mathbf{k}$ space, driven by the mean flow gradient. For purely homogeneous shear, this term is exact; for any inhomogeneity in the mean flow shear, the term is only a lowest order approximation to the Maclaurin expansion in $\mathbf{r}$. The entire expansion is, of course, highly complicated.

Even though term $(2 b)$ is exact to that order in $\mathbf{k}$ space, the corresponding term in $k$ space, modeled by the $c_{F}$ family of terms, cannot be expressed exactly except in circumstances of self-similarity of the spectral behavior. In the derivations of BHRZ the terms were modeled in a manner that relates modeling coefficients to those for the pressurevelocity coupling to mean flow. Heuristically we note that the overall structure for these modeled terms contains a form like

$$
\zeta\left[E_{i j}+\mu k \frac{\partial E_{i j}}{\partial k}\right],
$$

where $\mu$ is a dimensionless constant and $\zeta$ is a measure of the local mean flow strain rate. Note that this expression contains the lowest order terms from the expansion of

$$
\zeta E_{i j}[(1+\mu) k]
$$

as a power series in $\mu$. Thus, with no pretense at rigor, we are led to postulate a source term to $E_{i j}(\mathbf{x}, k)$ transport of the form

$$
-(1+\mu)\left\{E_{i n}[(1+\mu) k] \frac{\partial \bar{u}_{j}}{\partial x_{n}}+E_{j n}[(1+\mu) k] \frac{\partial \bar{u}_{i}}{\partial x_{n}}\right\}
$$

and to explore the possibility that this term can describe the vortex pairing process in mean-flow free shear. In effect, this term is meant to be a crude representation of a triad interaction, in which "turbulence" with components that are important as $k \rightarrow 0$ (the mean-flow free shear) couple with turbulence at wave number $(1+\mu) k$ to produce turbulence at $k$.

This vortex-pairing process appears to be quite distinct from that described in item 5 (spontaneous reverse cascade). The relationship to Leith's (1990) stochastic backscatter has not yet been worked out. 
The coefficient, $1+\mu$, in this nonlocal version of mean-flow turbulence creation is determined in such a way that the integral over all values of $k$ gives the same result as the creation rate with $\mu=0$ (i.e., the local version, which is exactly conservative of energy in exchange with the mean flow).

One can imagine generalizations of the terms

$$
c_{B} A_{i j}+c_{B 1} B_{i j}+c_{B 2} C_{i j}
$$

in which the $E_{i j}$ values are all located also at $(1+\mu) k$. The effective value of $\mu$ may vary from one type of term to another. For $\mu>0$ we purport to describe mean-flow-shear induced reverse cascade; for $\mu<0$ the induced cascade is direct. The turbulence at a given wave number may experience both. An eddy that is contracted in one direction may be expanded in an orthogonal direction. The easiest way to extract relevant information in this regard is to consider the mean-flow-induced cascade in the local formulation with the $c_{F}$ terms.

\section{Second-Point Cascade (Items 2 and 4)}

Kraichnan and Spiegel (1962) have suggested an interesting approximation to the transfer of energy in isotropic turbulence, which we refer to as second-point cascade. We shall discuss here some consequences of this approach and its relationship to the localized formulation.

The local cascade in $k$ space has been described by a combination of advective and diffusive terms:

$$
\frac{\partial E_{i j}(k, t)}{\partial t}=\frac{\partial}{\partial k}\left(-c_{1} k^{2} \sqrt{k E} E_{i j}+c_{2} k^{3} \sqrt{k E} \frac{\partial E_{i j}}{\partial k}\right) .
$$

We note that this form could be generalized to

$$
\begin{aligned}
\frac{\partial R_{i j}(\mathbf{k}, t)}{\partial t} & =\frac{\partial}{\partial k_{l}}\left(k_{l} g(k) R_{i j}(\mathbf{k})+k_{l} k_{n} f(k) \frac{\partial R_{i j}(\mathbf{k})}{\partial k_{n}}\right) \\
& =\frac{1}{k^{2}} \frac{\partial}{\partial k}\left(k^{3} g(k) R_{i j}(\mathbf{k})+k^{4} f(k) \frac{\partial R_{i j}(\mathbf{k})}{\partial k}\right) .
\end{aligned}
$$

Integration of this latter form over all angles in $\mathbf{k}$ space gives the following result:

$$
\frac{\partial E_{i j}(k, t)}{\partial t}=\frac{\partial}{\partial k}\left\{k[g(k)-2 f(k)] E_{i j}(k, t)+k^{2} f(k) \frac{\partial E_{i j}(k, t)}{\partial k}\right\} .
$$

When $g(k)=0$, we recover exactly our model form in $k$ space, with $c_{1} / c_{2}=2$, as mentioned in the Introduction and required for equilibrium of the equipartition spectrum. 
The essence of second-point cascade is exemplified by its formulation for $E_{\ell \ell}(k)$ or simply $E(k)$. Neglecting all the other contributions to the evolution of $E(k)$, we write the second-point terms as follows:

$$
\frac{\partial E(k, t)}{\partial t}=\int_{0}^{\infty} E(p) \Gamma(p, k) d p-\int_{0}^{\infty} E(k) \Gamma(k, p) d p .
$$

It follows that

$$
\frac{d}{d t} \int_{0}^{\infty} E(k, t) d k=0 .
$$

Dimensionality and equilibrium of the equipartition spectrum have been shown by Kraichnan and Spiegel (1962) to be strong constraints on the structure of the $\Gamma(p, k)$ function. If one writes the function as:

$$
\Gamma(p, k)=\Gamma_{0} \frac{p \sqrt{p E(p)}}{k}\left(\frac{k}{p}\right)^{n} g\left(\frac{p}{k}\right),
$$

and if $n$ is chosen to be $11 / 4$ and the weighting function, $g(\lambda)$, is chosen to equal $g(1 / \lambda)$ as well as to satisfy

$$
\begin{gathered}
g(x)<O\left(x^{-\frac{11}{4}}\right), \\
\int_{0}^{\infty} x^{\frac{7}{4}} g(x) d x=1 ;
\end{gathered}
$$

then the equipartition spectrum $E(k) \propto k^{2}$ will be an equilibrium spectrum. One may verify also that the inertial-range spectrum of Kolmogorov, $E(k) \propto k^{-\frac{5}{3}}$, will also be an equilibrium spectrum under the action of only inertial forces.

If one now inserts

$$
\Gamma(p, k)=\Gamma_{0}\left(\frac{k}{\bar{p}}\right)^{\frac{5}{4}} \sqrt{k E(p)} g\left(\frac{p}{k}\right)
$$

into the equation for $\partial E(k, t) / \partial t$, one finds that it can be written as:

$$
\frac{\partial E(k, t)}{\partial t}=\int_{0}^{\infty} d \lambda g(\lambda)\left[k^{\frac{3}{2}} E^{\frac{3}{2}}(k \lambda) \lambda^{-\frac{5}{4}}-k^{\frac{3}{2}} E^{\frac{3}{2}}(k) \lambda^{-\frac{15}{4}}\right],
$$

where we have replaced the variable, $p$, by $p=\lambda k$ and then replaced the new variable, $\lambda$, by $1 / \lambda$ in the second term. If one sets

$$
\Gamma(p, k)=\Gamma_{0} \frac{F(p, k)}{k} g\left(\frac{p}{k}\right),
$$

where

$$
F(p, k)=\left(\frac{k}{p}\right)^{\frac{5}{4}} k \sqrt{k E(p)}
$$


one finds the evolution equation of $E(k, t)$ is given by

$$
\frac{\partial E(k, t)}{\partial t}=\int_{0}^{\infty} d \lambda g(\lambda)\left[E(\lambda k) F(\lambda k, k)-\frac{E(k) F\left(k, \frac{k}{\lambda}\right)}{\lambda}\right] .
$$

When the width of $g(\lambda) \ll 1$, one can perform a Taylor series of the integrand about $\lambda=1$ and re-express the result in terms of the moments, $M_{m}$, of $g$ :

$$
M_{m}=\int_{0}^{\infty} d \lambda(\lambda-1)^{m} g(\lambda)
$$

We thereby obtain the following result, retaining only the first two moments,

$$
\frac{\partial E(k, t)}{\partial t}=\frac{\partial}{\partial k}\left\{M_{1} k^{\frac{5}{2}} E^{\frac{3}{2}}(k, t)+\frac{M_{2}}{2}\left[\frac{3}{2} k^{\frac{7}{2}} E^{\frac{1}{2}}(k, t) \frac{\partial E(k, t)}{\partial k}-6 k^{\frac{5}{2}} E^{\frac{3}{2}}(k, t)\right]\right\}
$$

which is conservative in $k$ space and satisfies the requirement that the inertial range spectrum (in the absence of inertial forces) is an equilibrium solution. For this local approximation to be valid, the first two moments must satisfy $M_{1}=3 M_{2} / 2$. To demonstrate this result, we first note that we can replace the variable $\lambda$ by $1 / \lambda$ in the expression of the moment, $M_{m}$, and thereby obtain

$$
M_{m}=(-)^{m} \int_{0}^{\infty}{\frac{(\lambda-1)^{m}}{\lambda^{m+2}}}^{m} g(\lambda) d \lambda
$$

However, $1 / \lambda^{m+2}$ can also be expressed as a Taylor series about $\lambda=1$ :

$$
\frac{1}{\lambda^{m+2}}=\sum_{n=0}^{\infty}(-)^{n}\left(\begin{array}{c}
m+n+1 \\
n
\end{array}\right)(\lambda-1)^{n}
$$

where the binomial coefficient is defined by:

$$
\left(\begin{array}{c}
m+n+1 \\
n
\end{array}\right) \equiv \frac{(m+n+1) !}{(m+1) ! n !}
$$

One thus obtains the following relation that must be satisfied by the moments of $g$ :

$$
M_{m}=\sum_{n=0}^{\infty}\left(\begin{array}{c}
m+n+1 \\
n
\end{array}\right)(-)^{m+n} M_{m+n}
$$

Keeping only the leading order moments appropriate in the limit of a narrow distribution, $g$, we retain:

$$
M_{m}=(-)^{m}\left(M_{m}-(m+2) M_{m+1}+\frac{(m+3)(m+2)}{2} M_{m+2}-\cdots\right) .
$$


This result pairs the moments in the following manner for a sufficiently narrow distribution, $g$ :

$$
M_{m}=\frac{(m+2)}{2} M_{m+1}, \quad m=\text { odd integer . }
$$

This also implies that an odd moment is always of the same order of magnitude as the next higher even moment. We utilize this result to insert $M_{1}=3 M_{2} / 2$ into our local approximation:

$$
\frac{\partial E(k, t)}{\partial t}=\frac{3 M_{2}}{2} \frac{\partial}{\partial k}\left[\frac{1}{2} k^{\frac{7}{2}} E^{\frac{1}{2}}(k, t) \frac{\partial E(k, t)}{\partial k}-k^{\frac{5}{2}} E^{\frac{3}{2}}(k, t)\right] .
$$

This yields the local Leith (1967) model that has the property that the equipartition spectrum is an equilibrium spectrum! We observe also that it has both wavelike and diffusive contributions.

If solutions of these equations settle down into generic forms that can be parameterized by, say, only two parameters, then, conceivably, in the presence of rapid transients the spectra can be modeled by linearly superposing these generic forms. If such proves to be the case, then only a finite number of parameters would be needed to model the evolution of rapid transients. These parameters could be determined by an equal number of spectral moments.

Returning to the integral form of the equation,

$$
\begin{aligned}
\frac{\partial E(k)}{\partial t} & =\int_{0}^{\infty} E(p) \Gamma(p, k) d p \\
& -\int_{0}^{\infty} E(k) \Gamma(k, p) d p,
\end{aligned}
$$

we can discuss its properties by an alternative reduction procedure based on a special form for the coupling function,

$$
\Gamma(p, k)=F(p, k) \delta(p-\lambda k)
$$

so that

$$
\Gamma(k, p)=F(k, p) \frac{1}{\lambda} \delta\left(p-\frac{k}{\lambda}\right) .
$$

After performing some algebraic reductions, we can return to a more general form of $\Gamma(p, k)$ by introducing an appropriate weighting function, $g(\lambda)$ and integrating over all possible values of $\lambda$ from 0 to $\infty$; the necessary relation is

$$
\frac{1}{k} g\left(\frac{p}{k}\right) F(p, k)=\Gamma(p, k) .
$$


Our second-point contribution to the evolution of $E(k)$ becomes, for a single second point at $k \lambda$,

$$
\begin{aligned}
\frac{\partial E(k)}{\partial t} & =E(\lambda k) F(\lambda k, k) \\
& -E(k) \frac{1}{\lambda} F\left(k, \frac{k}{\lambda}\right) ;
\end{aligned}
$$

and it is easily shown that

$$
\frac{\partial}{\partial t} \int_{0}^{k_{1}} E(k) d k=\int_{k_{1}}^{\lambda k_{1}} E(k) F\left(k, \frac{k}{\lambda}\right) \frac{d k}{\lambda} .
$$

Our goal, now, is to demonstrate the relation between second-point cascade and the local cascade ( $c_{1}$ and $c_{2}$ terms) of the BHRZ model. To accomplish the reduction of one to the other, we set $\lambda=1+\epsilon$ and expand as a power series in $\epsilon$. After considerable manipulation and replacing $\epsilon$ by $\lambda-1$, we find

$$
\begin{gathered}
\frac{\partial}{\partial t} \int_{0}^{k} E(k) d k=\frac{1}{\lambda}\left\{(\lambda-1) k_{1} E\left(k_{1}\right) F\left(k_{1}, k_{1}\right)\right. \\
\left.+\frac{(\lambda-1)^{2}}{2} k_{1}^{2}\left[F\left(k_{1}, k_{1}\right) \frac{\partial E\left(k_{1}\right)}{\partial k_{1}}+E\left(k_{1}\right)\left(F_{1}\left(k_{1}, k_{1}\right)-F_{2}\left(k_{1}, k_{1}\right)\right)\right]\right\}
\end{gathered}
$$

where

$$
\begin{aligned}
& F_{1}\left(k_{1}, k_{1}\right) \equiv\left[\frac{\partial F\left(\xi, k_{1}\right)}{\partial \xi}\right] \text { at } \xi=k_{1} \\
& F_{2}\left(k_{1}, k_{1}\right) \equiv\left[\frac{\partial F\left(k_{1}, \xi\right)}{\partial \xi}\right] \text { at } \xi=k_{1}
\end{aligned}
$$

Thus

$$
\begin{aligned}
\frac{\partial E(k)}{\partial t} & =\frac{\partial}{\partial k}\left\{\frac{\lambda-1}{\lambda} k\left[F(k, k)+\frac{\lambda-1}{2} k\left(F_{1}(k, k)+F_{2}(k, k)\right)\right] E(k)\right. \\
& \left.+\frac{(\lambda-1)^{2}}{2 \lambda} k^{2} F(k, k) \frac{\partial E(k)}{\partial k}\right\}
\end{aligned}
$$

plus terms proportional to $(\lambda-1)^{3}$. With proper choice to $F(p, k)$ this expression becomes the local cascade form with wave-like and diffusive contributions (the $c_{1}$ and $c_{2}$ terms in the BHRZ model). The diffusive term has a positive definite coefficient if $F(k, k)>0$. The wave-like part is negative for $\lambda<1$, describing (as expected) a rightward propagating wave in $k$ space; likewise for $\lambda>1$ the wave propagates to the left (i.e., lower wave numbers). Note that the diffusive part propagates energy toward both lower and higher wave numbers, whereas the basic second-point formulation is unidirectional for a given value of $\lambda$. It is tempting to give bidirectionality a physical interpretation, denoting the effects of both direct and reverse cascade; but this addition of physics is more reasonably associated with 
a weighted sum of contributions from many $\lambda$ values, both greater or less than unity. The contributions from $\lambda<1$ then represent the effects of large eddies "feeding" smaller ones in analogy to the feeding process from mean-flow shear; the contributions from $\lambda>1$ may then represent spontaneous eddy coalescence, especially when the turbulence is constrained to two space dimensions. (For three-dimensional turbulence this reverse process may violate some sort of generalized entropy constraint, but this speculation remains to be explored.)

Multiplication of the expanded equation by a weighting function, $g(\lambda)$, and integrating results in the form

$$
\begin{gathered}
\frac{\partial E(k)}{\partial t}=\frac{\partial}{\partial k}\left\{\left[N_{1} F(k, k)+N_{2} k\left(F_{1}(k, k)-F_{2}(k, k)\right)\right] k E(k)\right. \\
\left.+N_{2} k^{2} F(k, k) \frac{\partial E(k)}{\partial k}\right\}
\end{gathered}
$$

in which

$$
\begin{aligned}
& N_{1}=\frac{\int_{0}^{\infty} \frac{\lambda-1}{\lambda} g(\lambda) d \lambda}{\int_{0}^{\infty} g(\lambda) d \lambda} \\
& N_{2}=\frac{\int_{0}^{\infty} \frac{(\lambda-1)^{2}}{2 \lambda} g(\lambda) d \lambda}{\int_{0}^{\infty} g(\lambda) d \lambda} .
\end{aligned}
$$

A property of the BHRZ local-cascade model is that constancy of flux in both the $c_{1}$ and $c_{2}$ terms results in the classic $k^{-5 / 3}$ spectrum of the inertial regime. Does the secondpoint formulation retain this property? To answer this question consider the dimensionally proper expression

$$
F(\lambda k, k)=F_{0} k \sqrt{k E(\lambda k)}
$$

so that

$$
F\left(k, \frac{k}{\lambda}\right)=F_{0} \frac{k}{\lambda} \sqrt{\frac{k}{\lambda} E(k)} .
$$

Suppose that $E(k)$ and $E(\lambda k)$ both lie in a regime in which $E(k)=E_{0} k^{n}$. Then

$$
\frac{\partial E(k)}{\partial t}=E_{0}^{3 / 2} F_{0} k^{\frac{3}{2}(n+1)}\left(\lambda^{\frac{3 n}{2}}-\lambda^{-5 / 2}\right) \text {. }
$$

With $n=-5 / 3$ this expression vanishes, which thereby confirms that second-point cascade can preserve the classic behavior of the inertial regime.

We can see a constraint on $g(\lambda)$ when we return to the integral second-point form. If $g(\lambda) \equiv g_{0}$, a constant, then with the above choice for $F(p, k)$, we get

$$
\Gamma(p, k)=g_{0} F_{0} \sqrt{k E(p)}
$$


so that the integral second-point equation becomes

$$
\begin{aligned}
\frac{\partial E(k)}{\partial t} & =\int_{0}^{\infty} g_{0} F_{0} E(p) \sqrt{k E(p)} d p \\
& -\int_{0}^{\infty} g_{0} F_{0} E(k) \sqrt{p E(k)} d p
\end{aligned}
$$

which is nonconvergent. It follows that $g(\lambda)$ must be more locally compact around $\lambda=1$, with specific constraints that depend on the choice of $F(p, k)$.

Second-point cascade for $E_{i j}(k)$ is potentially somewhat richer in the choice of couplings from $\lambda k$ to $k$, with the coupling from $k$ to $k / \lambda$ following in conservative form. Whereas $E$ may have an inertial range with $k^{-5 / 3}$ spectral form, the deviator part, $\tilde{E}_{i j}$, is likely to fall off more rapidly in the inertial range, perhaps as $k^{-7 / 3}$ in many interesting circumstances.

The direct generalization for $E_{i j}(k)$ of the above form for $E(k)$ is

$$
\frac{\partial E_{i j}(k)}{\partial t}=\int_{0}^{\infty} E_{i j}(p) \Gamma(p, k) d p-\int_{0}^{\infty} E_{i j}(k) \Gamma(k, p) d p
$$

with the same (or another) form for $\Gamma(p, k)$. This form has the intuitively plausible justification that the transfer from $p$ depends directly on the strength of $E_{i j}$ at that wave number.

There is, however, a heuristic argument for another kind of second-point form. The guiding idea is based on the analogy to coupling from mean-flow shear, which, without modeling, can be written

$$
\frac{\partial E_{i j}(k)}{\partial t}=-E_{i \ell}(k) \frac{\partial \bar{u}_{j}}{\partial x_{\ell}}-E_{\ell j}(k) \frac{\partial \bar{u}_{i}}{\partial x_{\ell}} .
$$

Thus the source to $E_{i j}(k)$ is proportional to the various components of $E_{i j}$ at wave number $k$, not at the wave number of the source (the mean-flow shear). The magnitudes of $E_{i \ell}$ components associated with $\partial \bar{u}_{i} / \partial x_{\ell}$ are proportional to $\bar{u}_{\ell} \bar{u}_{\ell}$, so that we can estimate

$$
\left|\frac{\partial \bar{u}_{i}}{\partial x_{\ell}}\right| \approx k^{\prime} \sqrt{k^{\prime} E\left(k^{\prime}\right)}
$$

where $k^{\prime}$ is the wave number associated with the mean-flow free-shear-layer thickness. This line of reasoning then suggests second-point cascade coupling in the form

$$
\frac{\partial E_{i j}}{\partial t}=\int_{0}^{\infty} E_{i j}(p) \Gamma_{1}(k, p) d p-\int_{0}^{\infty} E_{i j}(k) \Gamma_{1}(p, k) d p
$$


with

$$
\Gamma_{1}(p, k)=\Gamma_{0}\left(\frac{p}{k}\right) p \sqrt{p E(p)}
$$

and $\Gamma_{0}\left(\frac{p}{k}\right)$ is a function similar to $g(\lambda)$ in describing the likely $p$ values that couple to $k$.

An even closer analogy can be made to the coupling to mean flow, in which we make the correspondence,

$$
\frac{\partial \bar{u}_{i}}{\partial x_{\ell}} \approx-k^{\prime} E_{i \ell}\left(k^{\prime}\right) \sqrt{\frac{k^{\prime}}{E\left(k^{\prime}\right)}}
$$

so that

$$
\begin{aligned}
\frac{\partial E_{i j}(k)}{\partial t} & =\int_{0}^{\infty} E_{i \ell}(p) E_{\ell j}(k) \Gamma_{2}(k, p) d p \\
& -\int_{0}^{\infty} E_{i \ell}(k) E_{\ell j}(p) \Gamma_{2}(p, k) d p \\
& +(i \leftrightarrow j),
\end{aligned}
$$

in which $(i \leftrightarrow j)$ means the same term with $i$ and $j$ interchanged, and

$$
\Gamma_{2}(p, k)=\Gamma_{0}\left(\frac{p}{k}\right) p \sqrt{\frac{p}{E(p)}}
$$

This more general second-point cascade form violates our idea of the separation of processes, in that it produces both a transfer in $k$ space and a redistribution of the tensor components. Its close analogy to mean-flow coupling, however, furnishes good incentive for investigating the properties of this model.

Each of these models can be reduced to a corresponding single-second-point form by setting

$$
\Gamma_{0}\left(\frac{p}{k}\right)=\gamma_{0} \delta\left(\frac{p}{k}-\lambda\right)
$$

and by expanding in a power series in $(\lambda-1)$, we can obtain a local approximation for each of these forms.

\section{Nonlocal Rates (Item 6)}

Another class of nonlocality has local processes driven by nonlocal rates. Return to isotropy, for example in the Rotta formulation, is written

$$
\frac{\partial E_{i j}(k)}{\partial t}=c_{M} k \sqrt{k E(k)}\left[\frac{1}{3} E(k) \delta_{i j}-E i j(k)\right] \text {. }
$$

To lowest order, the local return to isotropy is proportional to the local departure therefrom, with a rate coefficient that depends only on the strength of turbulence at that wave number. Many investigators have recognized, however, that actions disruptive 
to variations in strength among the turbulence components come from the randomizing effects of the turbulence at all scales. Various alternative rate coefficients have accordingly been proposed, of which two types are

$$
\begin{aligned}
& \frac{\partial E_{i j}(k)}{\partial t}=c_{M} \int_{0}^{k_{1}} f\left(\frac{k^{\prime}}{k}\right) \sqrt{k^{\prime} E\left(k^{\prime}\right)} d k^{\prime}\left[\frac{1}{3} E(k) \delta_{i j}-E_{i j}(k)\right] \\
& \frac{\partial E_{i j}(k)}{\partial t}=c_{M} \sqrt{\int_{0}^{k_{1}} f\left(\frac{k^{\prime}}{k}\right)\left(k^{\prime}\right)^{2} E\left(k^{\prime}\right) d k^{\prime}}\left[\frac{1}{3} E(k) \delta_{i j}-E_{i j}(k)\right],
\end{aligned}
$$

in which $k_{1}=k$ or $k_{1}=\infty$, and $f\left(\frac{k^{\prime}}{k}\right)$ is a dimensionless function of its argument. Different forms emphasize the effects of different parts of the spectrum. Performing the integral before taking the square root, for example, gives an enhanced effect from the dominant parts of the $E(k)$ spectrum; this form was suggested by Herring (1993), with $f\left(\frac{k^{\prime}}{k}\right)=1$.

Another type of nonlocal rate occurs in the expressions for second-point cascade. In exploiting the analogy to mean-flow shear coupling, we represented the nature of $\partial \bar{u}_{i} / \partial x_{\ell}$ in terms of $E_{i \ell}$ at a single wave number. Actually, a more effective representation would carry some measure of the full spectral richness of the large-eddy analogy to free shear, so that a variety of integral representations for $F(\lambda k, k)$ could be proposed, much like those of the rate coefficient for return to isotropy. Even when the second-point formulation has been expanded as a power series in $(\lambda-1)$, the nonlocality can be conceived as persisting. Thus, the simplest version of this representation,

$$
\frac{\partial E_{i j}(k)}{\partial t}=\frac{\partial}{\partial k}\left[-c_{1} k^{2} \sqrt{k E(k)} E_{i j}(k)+c_{2} k^{3} \sqrt{k E(k)} \frac{\partial E i j(k)}{\partial k}\right],
$$

could be extended to

$$
\frac{\partial E_{i j}(k)}{\partial t}=\frac{\partial}{\partial k}\left[-c_{1} \int_{0}^{k_{1}} k^{\prime} \sqrt{k^{\prime} E\left(k^{\prime}\right)} d k^{\prime} E_{i j}(k)+c_{2} \int_{0}^{k_{1}}\left(k^{\prime}\right)^{2} \sqrt{k^{\prime} E\left(k^{\prime}\right)} d k^{\prime} \frac{\partial E i j(k)}{\partial k}\right]
$$

and numerous variants, including the presence of $f\left(\frac{k^{\prime}}{k}\right)$ in the integrands and a version with integration performed before taking the square root.

The effective eddy viscosity (turbulence diffusion coefficient) is also an excellent candidate for nonlocal representation. The turbulence self-diffusion represents a very complicated combination of random-walk processes, derived from the stochastic effects of fluctuations from all scales. With the inclusion of passive or active materials in the fluid, the stochastic advective transport may include an ordered (wave-like) interpenetration as well as the disordered (diffusive) part [Steinkamp, Clark, and Harlow (1995)]. 
The most effective part of the spectrum for the accomplishment of diffusion is associated with the largest scales of fluctuating motion. Indeed it is on this basis that the meaningful definition of single-point dissipation (associated with $\epsilon_{i j}$, the dissipation tensor) is related to the flux of spectral Reynolds stress components from the small-wavenumber (large-scale) part of the spectrum to the large-wave-number (small-scale) part of the spectrum, rather than the viscous dissipation from small scales to heat.

Heuristic justification for the dominant importance of small-wave-number fluctuations is based on the random-walk formula for the mean expected displacement, $D$, that results from $N$ steps of individual displacement with speed $v$ acting over a time increment, $\delta t$,

$$
D=v \delta t \sqrt{N} .
$$

Over a total elapsed time, $T$, we note that $\delta t=T / N$, so that

$$
D=\frac{v T}{\sqrt{N}} .
$$

For a fixed $v$ and a given total elapsed time, the fewer the steps the greater the expected displacement. Because each step is of length $v T / N$, we conclude that the larger the individual step the greater is $D$. For turbulence this means that the larger scales will give much more displacement in a given period of time than will be produced by the small scales.

The simplest local representation for the turbulence diffusion coefficient, $\nu_{t}$, is derived purely on the basis of dimensionality,

$$
\nu_{t}=c_{D} \sqrt{\frac{E(k)}{k}}
$$

although the relationship to the formula for $D$ is clearly visible through the relation $D=\sqrt{\nu_{t} T}$. Rewriting the random-walk formula in the form $D=\sqrt{v T \delta x}$, with $\delta x$ being the displacement per step, we are led to associate

$$
\nu_{t}=v \delta x
$$

The correspondence with turbulence is $v \rightarrow \sqrt{k E}$ and $\delta x \rightarrow 1 / k$, which leads to the expression for $\nu_{t}$ as moderated by the dimensionless constant, $c_{D}$.

In BHRZ a nonlocal extension for this coefficient is suggested in the form

$$
\nu_{t}=c_{D} \int_{0}^{\infty} \frac{\sqrt{k E(k)}}{k^{2}} d k
$$


Extending the integration to $k=\infty$ sums contributions from wave numbers both smaller and larger than the particular wave number of consideration. Can the contributions for large wave numbers be considered in analogy to the Brownian diffusion of particles by bombardment with molecules that are much smaller?

More general variants for $\nu_{t}$ include

$$
\nu_{t}(k)=\int_{0}^{k_{1}} f\left(\frac{k}{k^{\prime}}\right) \sqrt{k^{\prime} E\left(k^{\prime}\right)} \frac{d k^{\prime}}{\left(k^{\prime}\right)^{2}}
$$

and the extended Herring version,

$$
\nu_{t}(k)=\sqrt{\int_{0}^{k_{1}} f\left(\frac{k^{\prime}}{k}\right) E\left(k^{\prime}\right) \frac{d k^{\prime}}{\left(k^{\prime}\right)^{2}}}
$$

There is another variant to turbulent diffusion that is also potentially quite significant, namely the introduction of nonisotropic diffusion with a tensor diffusion coefficient, such that

$$
\frac{\partial E_{i j}}{\partial t}=\frac{\partial}{\partial x_{\ell}} \nu_{t, \ell m} \frac{\partial E_{i j}}{\partial x_{m}} .
$$

In violation of our postulate of the separation of processes, we could imagine a variety of versions in which the diffusive flux of $E_{i j}$ is driven by gradients of other components of the tensor. There are numerous variants, for example,

$$
\frac{\partial E_{i j}}{\partial t}=\frac{\partial}{\partial x_{\ell}} \nu_{t, i m} \frac{\partial E_{\ell j}}{\partial x_{m}}+(i \leftrightarrow j),
$$

and even

$$
\frac{\partial E_{i j}}{\partial t}=\frac{\partial}{\partial x_{i}} \nu_{t, \ell m} \frac{\partial E_{\ell j}}{\partial x_{m}}+(i \leftrightarrow j) .
$$

The proper choice among these possibilities is discussed in Chapter V. 


\section{NONLOCAL PROCESSES IN PHYSICAL SPACE}

Correlations between points that are separated in physical space enable the characterization of two closely related nonlocal processes:

- The local (in physical space) interactions among structures at various scales (nonlocal in wave-number space).

- The instantaneous pressure-wave propagation of fluctuations from one point in physical space to another (nonlocal in physical space).

The first of these is discussed in Chapter II. The second is discussed in this chapter.

For a fluid with constant density, the two processes that concern us in physical space are wave-like propagation, especially related to the nonlocal coupling between turbulence and mean flow, and the diffusion-like propagation arising from nonlocal coupling among turbulence structures at different positions. Again, we exploit the close relationship between two types of interactions, one of them with large structures that can be considered mean flow and the other with large structures that are considered to be the low-wavenumber parts of the turbulence itself. The mean-flow-coupling part arises directly from the derivations and is easily expressed in the transport equations; the large-scale coupling emerges through the triple-correlation functions and is accordingly more obscure insofar as effective modeling is concerned, but is amenable to modeling guidance by analogy to the more transparent formulations for the mean-flow coupling.

Modeling these nonlocal processes also receives much valuable assistance by the use of generic self-similar form functions, as discussed in Chapter IV. In essence, the concept of these form functions takes its validity from the continuous tendency for turbulence to adapt to its surroundings in ways that are highly constrained by the fundamental principles of available tensor forms, dimensionality, Galilean invariance, and by the ideas of generalized entropy (this last being described by realizability in its extremes of manifestation).

Universal self-similar forms are at best an approximation to the real structure, and there are significant questions regarding their existence in rapidly changing external drive circumstances. Even in seemingly ideal surroundings there are issues of nonuniqueness that arise from the persistence of perturbations in the very-low-wave-number parts of the spectrum. But a particularly useful aspect of these form functions is that they do not require homogeneity for their existence; indeed the most significant and valuable form functions are those that arise with strongly nonhomogeneous, nonisotropic drivers, for example in free shear layers, strong mean-flow pressure gradients, the effects of nearby rigid walls, and the existence of large gradients in density. 
The classic example of generic form occurs in the idealized circumstances of homogeneous isotropic turbulence decay. The self-similar aspects are expressed by

$$
E(k, t)=K(t) L(t) F[k L(t)]
$$

in which $F$ is suitably normalized so that $K(t)$ is the total turbulence energy per unit mass and $L(t)$ is a length scale for the dominant parts of the turbulence spectrum. BHRZ showed that $F$ is easily determined, has a $k^{-5 / 3}$ inertial range as predicted by Kolmogorov, and most especially is of great value for taking moments of the spectral transport equations to derive single-point models in the $R_{i j}-\epsilon_{i j}$ and $k-\epsilon$ forms.

It is the use of these forms for taking moments that makes them relevant to the considerations of this chapter. Nonlocal processes in physical space arise most prominently from the integral solution for pressure that appears in the pressure-velocity correlations. The generic forms can help us to derive expressions for these integrals that are highly tractable for modeling.

An especially important nonlocal interaction in physical space is the remote coupling of turbulence creation to mean-flow gradients. We also discuss in this chapter the remote coupling of small-scale structures to large-scale structures, manifested as a nonlocal contribution to the turbulence self-diffusion.

The coupling of turbulence to mean flow is usually described by local terms that conserve the overall transfer of energy from mean-flow shear to the fluctuations. In $|\mathbf{k}|$ space, the nonconservative coupling part is

$$
\frac{\partial E_{i j}}{\partial t}=-E_{i n} \frac{\partial \bar{u}_{j}}{\partial x_{n}}-E_{n j} \frac{\partial \bar{u}_{i}}{\partial x_{n}},
$$

where the terms are all at position $\mathbf{x}$ and scalar wave number $k$. In addition there are conservative coupling terms, the $c_{B}$ and $c_{F}$ terms, which can also be written in local form (i.e., at position $\mathbf{x}$ ). For homogeneous shears and for fine scale turbulence in a free shear layer of width $W$ (such that $k W>1$ ), the local coupling expressions are good approximations. For $k W<1$, however, the approximations are poor.

Suppose, first that we consider only the nonconservative terms and are dealing with a homogeneous shear, say $\overline{\mathbf{u}}=(U y / W) \mathbf{x}_{1}$. Then $\bar{u}_{i, j} \equiv \partial \bar{u}_{i} / \partial x_{j}=U / W \delta_{i 1} \delta_{j 2}$. Without the conservative terms, the above equation cannot have a solution that is exponential in time, say of the form $E_{i j}=E_{i j}^{0} \exp (\omega t)$, which is expected from the classic Kelvin-Helmholtz $(\mathrm{KH})$ analysis for wave numbers small compared with $W^{-1}$. If it did, we would find that

$$
-\omega E_{i j}^{0}=-\frac{U}{W}\left(E_{i n}^{0} \delta_{j 1} \delta_{n 2}+E_{n j}^{0} \delta_{i 1} \delta_{n 2}\right) .
$$


But this would imply that

$$
\begin{aligned}
& -\omega E_{11}^{0}=-\frac{2 U}{W} E_{12}, \\
& -\omega E_{12}^{0}=-\frac{U}{W} E_{22}, \\
& -\omega E_{22}^{0}=0 .
\end{aligned}
$$

Hence there is no nontrivial time-exponential solution. Indeed one can show that the solution of this equation is given by

$$
E_{i j}(t)=E_{i j}^{0}-\left(E_{i 2}^{0} \delta_{j 1}+E_{2 j}^{0} \delta_{i 1}\right) \frac{U t}{W}+E_{22}^{0} \delta_{i 1} \delta_{j 1}\left(\frac{U t}{W}\right)^{2} .
$$

The production of an exponential instability must depend on other terms. If we restrict ourselves to including solely the $c_{B}$-terms, we obtain the following set of coupled equations:

$$
\begin{aligned}
& \frac{\partial E_{11}}{\partial \tilde{t}}=\left(\frac{4}{3} c_{B}-\frac{2}{3} c_{B_{1}}-2\right) E_{12}, \\
& \frac{\partial E_{22}}{\partial \tilde{t}}=\left(\frac{4}{3} c_{B_{1}}-\frac{2}{3} c_{B}\right) E_{12}, \\
& \frac{\partial E_{33}}{\partial \tilde{t}}=-\frac{2}{3}\left(c_{B}+c_{B_{1}}\right) E_{12}, \\
& \frac{\partial E_{12}}{\partial \tilde{t}}=\left(c_{B}-1+c_{B_{2}}\right) E_{22}+\left(c_{B_{1}}+c_{B_{2}}\right) E_{11}+c_{B_{2}} E_{33},
\end{aligned}
$$

where $\tilde{t}=U t / W$. We use the values $c_{B_{1}}=8 c_{B}-6, c_{B_{2}}=-3 c_{B}+11 / 5$, and $c_{B}=16 / 21$. If one takes the second time derivative of the last equation and then utilizes the first three equations, one finds the following results:

$$
\begin{aligned}
& \frac{\partial^{2} E_{12}}{\partial \tilde{t}^{2}}=0.162 E_{12}, \\
& \frac{\partial E_{11}}{\partial \tilde{t}}=-1.048 E_{12}, \\
& \frac{\partial E_{22}}{\partial \tilde{t}}=-0.381 E_{12}, \\
& \frac{\partial E_{33}}{\partial \tilde{t}}=-0.571 E_{12} .
\end{aligned}
$$

These equations have exponentially growing solutions, varying as $\exp (0.403 \mathrm{Ut} / \mathrm{W})$. For consistency, all that is required is (as is seen to be the case) that the signs of the right-hand sides of the last three equations must all be the same. The crucial point, however, is that the growth rate is independent of wave number, in contradiction to $\mathrm{KH}$ analysis. 
We can also include the effects of the $c_{F}$ terms in this analysis, although they represent mode coupling, which is not present in the linearized Navier-Stokes equations. With the spectral behavior varying at $k^{n}$ for small wave numbers, the result for the square of the exponential growth coefficient is

$$
\begin{gathered}
\frac{1}{3}\left\{\left(c_{B}-1\right)\left[\left(27 c_{B}-20\right)+\left(2-3 c_{B}\right) n\right]+\left(8 c_{B}-6\right)(n+5)\left(2-3 c_{B}\right)-\right. \\
\left.\frac{1}{5}\left[\left(21 c_{B}-20\right)+\left(21 c_{B}-16\right) n\right]\right\} \\
+\frac{1}{3}(n+1)\left\{\left[1-\frac{3}{2} c_{B}\right]\left[(2 n+5)\left(2-3 c_{B}\right)+\left(27 c_{B}-20\right)\right]-\right. \\
\left.\frac{1}{20}\left[\left(21 c_{B}-20\right)+\left(21 c_{B}-16\right) n\right]\right\} .
\end{gathered}
$$

For the specific case of $c_{B}=16 / 21$ and $n=2$, the solution thus varies as $\exp (0.83 U t / w)$. In contrast to the $\mathrm{KH}$ solution of the linearized Navier-Stokes equations in which there is no node coupling, the model equations with purely local coupling show sensitivity to spectral form in a manner that is independent of wave number.

In contrast, the $\mathrm{KH}$ solution for free-shear-layer instability growth of velocity amplitude, $A$, is

$$
A=A_{0} \exp \left(\frac{1}{2} k U t\right),
$$

which is valid for as long as $k W \ll 1$, so that the fluctuational energy content per unit mass in the shear layer is

$$
E=E^{0} \exp (k U t)
$$

The basis for the error in turbulence transport analysis lies in the neglect of nonlocal processes. As shown by the $\mathrm{KH}$ analysis, the velocity potential extends into the far lateral regions beyond the shear itself, varying as $e^{-k|y|}$, where $y$ is the normal coordinate with origin in the shear layer. Both analyses drive the fluctuations with the same force, but the KH derivation contains the inertial resistance of adjacent mass well outside the shear layer whereas the local turbulence transport derivation does not include this effect. The total fluctuational energy is the same; the difference between the two within the shear layer manifests two very different energy distributions, one of them nonlocal and the other local.

The nonlocal coupling between turbulence and mean flow is even more visibly manifested in the flow past an obstacle. At low Reynolds numbers $(\sim 100$ to several 
thousands) the von Karman vortex street is a prominent feature for an obstacle that is of infinite spanwise extent.

The fluctuations in flow velocity, which at all scales can be considered to represent turbulence, extend far above and below the downstream shadow of the obstacle. They occur in those lateral regions because of the instantaneous propagation of pressure waves to distances from the mean-flow shear layers that are an appreciable fraction of the dominant wave length. Inclusion of these effects in the analysis of turbulence means that valid transport equations for their representation must describe this strongly nonisotropic spectral structure at low magnitudes of wave number, again indicating the necessity for nonlocal coupling between the turbulence and the mean flow.

Thus purely local modeling of mean-flow coupling in turbulence transport equations can result in a completely fallacious treatment of the low-wave-number parts of the spectrum, in regards to both Reynolds stress magnitude and lateral extent of propagation. Issues regarding form-function structure and uniqueness can be obscured by neglect of the nonlocality, with significant consequences for validity of the single-point models that are a major focus for this work.

Some of these issues of nonlocality have been discussed by Naot, Shavit, and Wolfshtein (1973), Ohkitani and Kishiba (1995), and Demuren, Lele, and Durbin (1994). We follow a somewhat different, more heuristic, approach.

As useful guidance for development of the nonlocal coupling terms in turbulence transport, we consider the full KH solution for the stability of incompressible flow with a thin free shear layer $(k W \ll 1)$. In particular we summarize results of a stability analysis of a fluid of density $\rho$ having an equilibrium velocity $\mathbf{U}(\mathbf{r})=\hat{\mathbf{x}} \operatorname{sign}(y) U_{0} / 2$. Thus at the plane $y=0$, the flow velocity has an abrupt jump of $U_{0}$.

It follows from Kelvin's circulation theorem that this perturbed flow must be a potential flow. Because the geometry of the equilibrium is spatially homogeneous in $x$, it suffices to analyze the linear stability of the flow in terms of infinitesimal perturbations having the functional dependence, $\exp (i k x+\gamma t)$, where $k$ and $\gamma$ are the wave number and growth rate, respectively. We set $\mathbf{u}^{\prime}=-\nabla \phi^{\prime}$, where $\phi^{\prime}=\phi(y) \exp (i k x+\gamma t)$. Because our flow is incompressible, $\nabla^{2} \phi^{\prime}=0$. To satisfy the boundary conditions of the perturbation velocities vanishing at $y= \pm \infty$, we find that

$$
\begin{aligned}
& \phi(y)=\phi_{+0} \exp (-k y), y>0 \\
& \phi(y)=\phi_{-0} \exp (+k y), y<0 .
\end{aligned}
$$


To obtain the relations between $\phi_{-0}$ and $\phi_{+0}$, we observe that the perturbed flow $\mathbf{u}^{\prime}$ must satisfy

$$
\frac{\partial \mathbf{u}^{\prime}}{\partial t} \pm \frac{U_{0}}{2} \frac{\partial \mathbf{u}^{\prime}}{\partial x}=-\nabla p_{ \pm}^{\prime},
$$

in which $p_{ \pm}^{\prime}$ represents the perturbed pressure above and below the discontinuity. Inserting the assumed time and space dependences for the perturbed quantities, we obtain:

$$
\left(\gamma \pm i k \frac{U_{0}}{2}\right) \phi_{ \pm 0}=p_{ \pm 0}
$$

where the perturbed potential and pressure amplitudes above and below the discontinuity are given by $p_{ \pm 0}$ and $\phi_{ \pm 0}$, respectively. Pressure balance across the surface requires continuity of the pressure perturbations, $p_{+0}+=p_{-0}$, so that

$$
\left(\gamma+i k \frac{U_{0}}{2}\right) \phi_{+0}=\left(\gamma-i k \frac{U_{0}}{2}\right) \phi_{-0} .
$$

Thus the potential amplitudes just above and below differ merely by a phase factor. We shall denote their squared magnitude by $\left|\phi_{0}\right|^{2}$.

Assuming the perturbed surface is described by $y_{s}(x, t)=\eta \exp (i k x+\gamma t)$ and taking the convective derivative of $y_{s}$, we find that the vertical motion of the fluid is given by

$$
\begin{aligned}
& \left(\gamma+i k \frac{U_{0}}{2}\right) \eta=-\left.\frac{\partial \phi_{+}}{\partial y}\right|_{y=0}=k \phi_{+0} \\
& \left(\gamma-i k \frac{U_{0}}{2}\right) \eta=-\left.\frac{\partial \phi_{-}}{\partial y}\right|_{y=0}=-k \phi_{-0} .
\end{aligned}
$$

If one solves for $\phi_{ \pm 0}$ in terms of $\eta$ and inserts it into the pressure balance constraint, one immediately obtains the dispersion relation:

$$
\gamma= \pm \frac{k U_{0}}{2} .
$$

To describe growing modes, we choose only the positive sign. For these modes, we note that $\phi_{+0}=-i \phi_{-0}$.

With these results, we can evaluate the averages of the second-order quantities, $u_{i}^{\prime}(x, y, t) u_{j}^{\prime}(x, y, t)$, by defining the average of any quantity, say $Q(x, y, t)$, by

$$
\bar{Q}(y, t)=\frac{k}{2 \pi} \int_{0}^{\frac{2 \pi}{k}} d x Q(x, y, t) .
$$

We thereby obtain:

$$
\overline{\mathbf{E}}(y, t)=\frac{\left|\phi_{0}\right|^{2}}{4}\left\{\begin{array}{lll}
1 & 0 & 0 \\
0 & 1 & 0 \\
0 & 0 & 0
\end{array}\right\} \exp (-2 k|y|+2 \gamma t) .
$$


It is interesting to note that there is no net transport of tangential momentum normal to the interface in this low-amplitude perturbation-analysis result.

To illustrate the formalism that supports nonlocal modeling as an important factor for effective turbulence transport representations, we examine one isolated part of the transport equation for single-point $R_{i j}$, namely

$$
\frac{\partial R_{i j}}{\partial t}=\frac{1}{\rho} \overline{p^{\prime}\left(\frac{\partial u_{i}^{\prime}}{\partial x_{j}}+\frac{\partial u_{j}^{\prime}}{\partial x_{i}}\right)} .
$$

For pressure behavior, the Poisson equation

$$
\frac{\partial^{2} p}{\partial x_{n}^{2}}=-\frac{\partial u_{i}}{\partial x_{j}} \frac{\partial u_{j}}{\partial x_{i}}
$$

is useful to illustrate our point. This equation has the solution over an infinite domain

$$
p=-\frac{\rho}{4 \pi} \int \frac{\left(\frac{\partial u_{n}}{\partial x_{\ell}} \frac{\partial u_{\ell}}{\partial x_{n}}\right)_{\mathbf{x}_{1}}}{\left|\mathbf{x}-\mathbf{x}_{1}\right|} d \mathbf{x}_{1}
$$

so that

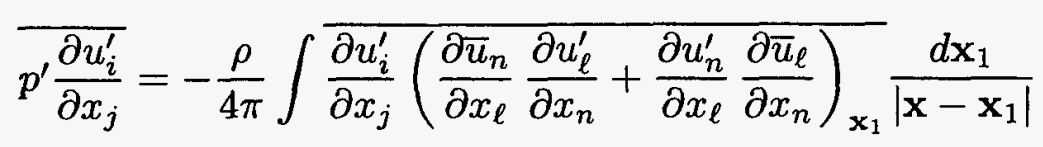

plus triple-correlation terms. With integration by parts and some rearrangement, this result can be inserted into the $R_{i j}$ equation to give

$$
\frac{\partial R_{i j}(\mathbf{x})}{\partial t}=-\frac{1}{2 \pi} \int \frac{\partial \bar{u}_{m}\left(\mathbf{x}_{1}\right)}{\partial x_{1 m}}\left[\frac{\partial R_{i n}\left(\mathbf{x}, \mathbf{x}_{1}\right)}{\partial x_{j}} \frac{\partial}{\partial x_{1 m}}\left(\frac{1}{\left|\mathbf{x}-\mathbf{x}_{1}\right|}\right)\right] d \mathbf{x}_{1}
$$

plus a symmetrizing term and some triple-correlation terms.

Thus the nonlocal coupling of turbulence to mean-flow gradients is demonstrated to occur through a weighted interaction of the two-point Reynolds stress with the meanflow shears at all points in physical space surrounding $\mathbf{x}$. This nonlocal coupling, arising from correlations of pressure fluctuations with those of the velocity gradients, describes a completely energy-conserving redistribution of Reynolds stress components created by interactions with the mean-flow shears. It is supplementary to the purely local coupling terms that arise from momentum advection. The triple-correlation terms, omitted in the above derivation, also contribute nonlocal effects, which can be interpreted in terms of turbulence-induced propagation of turbulence, often represented as turbulence selfdiffusion, but also including the processes of return to isotropy and cascade in wave-number space. 
Thus we see that the derivation of turbulence transport representations for nonlocal source terms in physical space are most easily accomplished by means of the two-point representation, without the Fourier transform to $\mathbf{k}$ space. [Oberlack and Peters (1993) have noted a variety of advantages for working in $\mathbf{x}, \mathbf{x}_{2}$ space or $\mathbf{x}, \mathbf{r}$ space.] The direct transformation to wave-number space does not result in forms that have proved intuitively meaningful to us or convenient for implementation into fluid-dynamics computer codes. We could remedy this difficulty by two methods. One is to postulate a conservative redistribution of Reynolds stress components based on the results of the $\mathrm{KH}$ analysis presented above. The second is to insert appropriate generic form functions into the integral expression in order to reduce the expression to tractability. We follow the first approach, with the second one being described qualitatively in Chapter IV.

We note that BHRZ derive the local coupling to mean-flow shear by setting part of the Poisson-solution integrand at position $\mathbf{x}_{1}$ to the value at position $\mathbf{x}$. Higher-order corrections might be imagined through an expansion of the integrand as a power series in $\mathbf{x}_{1}-\mathbf{x}$, but this may not be a convergent process.

Guided by the above derivation and by our postulate of separation of processes, we formulate a description for spatial redistribution of Reynolds stress components in a way that does not re-order their relative strengths or transport them in $k$ space by this same mechanism. In scalar wave-number space we consider expressions of the type

$$
\frac{\partial E_{i j}(\mathbf{x}, k, t)}{\partial t}=\int G_{i j}\left(\mathbf{x}^{\prime}, k, t\right) Q\left(\mathbf{x}^{\prime}, \mathbf{x}\right) d \mathbf{x}^{\prime},
$$

in which $G_{i j}\left(\mathbf{x}^{\prime}, k, t\right)$ is the purely local coupling source at $\mathbf{x}^{\prime}$, and $Q\left(\mathbf{x}^{\prime}, \mathbf{x}\right)$, which also depends on $k$, redistributes that source from $\mathbf{x}^{\prime}$ to $\mathbf{x}$. Conservation requires

$$
\int Q\left(\mathbf{x}^{\prime}, \mathbf{x}\right) d \mathbf{x}=1
$$

With local coupling, $Q\left(\mathbf{x}^{\prime}, \mathbf{x}\right)=\delta\left(\mathbf{x}^{\prime}-\mathbf{x}\right)$. In the vicinity of a free shear layer, the $\mathrm{KH}$ solution suggests $Q\left(\mathbf{x}^{\prime}, \mathbf{x}\right)$ decreases as $\exp (-2 k|y|)$, where $|y|$ is the distance from the layer.

To examine the properties of such a formulation, we therefore postulate

$$
Q\left(y^{\prime}, y\right)=k \exp \left[-2 k\left|y^{\prime}-y\right|\right]
$$

everywhere. For large wave numbers, i.e., $k W>1$, this form gives essentially the local behavior, as appropriate. It is thus effective in broadening the distribution only for small wave numbers, i.e., for structures large compared to the shear-layer width. Does this 
broadening take place in a correct manner? A significant criterion lies in whether or not its effect is to recapture the $\mathrm{KH}$ linear mode-growth behavior at small wave numbers. To demonstrate that this is, indeed, the case, we return to the evolution equation for $E_{i j}$. As before, we set

$$
E_{i j}=E_{i j}^{0}(y) f(t)
$$

and nondimensionalize the mean-flow velocity gradient with $U / W$, so that with nonlocal creation

$$
E_{i j}^{0}(y) \frac{d f}{d t}=-k f(t) \frac{U}{W} \int_{-\infty}^{\infty} S_{i j}(y) e^{-2 k\left|y^{\prime}-y\right|} d y^{\prime},
$$

in which $S_{i j}(y)$ is the entire nondimensionalized coupling source. We can accomplish our demonstration by approximating the integral for $y>W / 2$ as follows:

$$
\int_{-\frac{W}{2}}^{\frac{W}{2}} \frac{1}{2} S_{i j}(0) e^{-2 k\left(y-y^{\prime}\right)} d y^{\prime}=\frac{1}{4} S_{i j}(0) e^{-2 k y}\left(e^{k W}-e^{-k W}\right) .
$$

Note that $S_{i j}(0)$ overestimates the integral while $S_{i j}\left(\frac{W}{2}\right)$ underestimates it. We use $\frac{1}{2} S_{i j}(0)$ as a best guess so that

$$
E_{i j}^{0}(y) \frac{d f}{d t}=-\frac{1}{4} f(t) \frac{U}{W} S_{i j}(0) e^{-2 k y}\left(e^{k W}-e^{-k W}\right) .
$$

Twice the integral from $W / 2$ to $\infty$ thus gives the total amount of $E_{i j}$ created per unit time outside of the shear layer, which accordingly is

$$
2 \int_{\frac{W}{2}}^{\infty} E_{i j}(y) \frac{d f}{d t} d y=-f(t) U S_{i j}(0) \frac{1-e^{-2 k W}}{4 k W}
$$

The total amount created per unit time everywhere is (because of the normalization of $Q$ ) the same as the local-theory total amount, namely

$$
\frac{U}{W} f(t) S_{i j}(0) W .
$$

Thus the nonlocal prediction for total $E_{i j}$ created inside the shear layer per unit time is

$$
\frac{\partial E_{i j} \text { (inside) }}{\partial t}=-U f(t) S_{i j}(0)\left[1-\frac{1-e^{-2 k W}}{2 k W}\right]
$$

or, continuing the approximation,

$$
W E_{i j}^{0}(0) \frac{d f}{d t}=-U f(t) S_{i j}(0)\left[1-\frac{1-e^{2 k W}}{2 k W}\right],
$$


so that

$$
\frac{d f}{d t}=\frac{N U}{W} f\left[1-\frac{1-e^{-2 k W}}{2 k W}\right],
$$

in which $N$ is a constant numerical factor. Thus

$$
f=f_{0} \exp \left\{\frac{N U t}{W}\left[1-\frac{1-e^{-2 k W}}{2 k W}\right]\right\},
$$

which shows the nonlocal modification to the exponential growth rate. For $k W \gg 1$ the modification is small (and could be made even smaller with an appropriate change to the form of $Q$ ). For $k W \ll 1$ we note that

$$
f=f_{0} \exp (N U k t)
$$

which, with $N=0.5$, has the correct wave-number variations as in the linear KelvinHelmholtz solution.

This procedure for capturing the low-wave-number effects of nonlocality in physical space can also be applied to circumstances with large density variations. As shown by Steinkamp, Clark, and Harlow (1995), the classic Rayleigh-Taylor instability can be described by a turbulence transport model with a similar spatial redistribution of Reynolds stress creation terms. 


\section{SELF-SIMILAR FORMS}

\section{A. Introductory Description}

BHRZ showed that the concept of approximate self-similarity is a crucial element for the validity of single-point turbulence transport models. In order to describe with a small number of variables the collective effects of a virtually infinite number of degrees of freedom, it is necessary that severe constraints exist to confine the dynamics of the fluid to a very small set of all possibilities. Moreover, it is necessary that the approach to approximate self-similarity occurs rapidly after a sudden change in the external drive conditions. (There are direct analogies to these considerations in assessing the validity of the Navier-Stokes equations for describing the collective dynamics of numerous molecules.)

In anticipation of ubiquitous occurrence of self-similar forms, we also refer to them as generic forms. The simplest type of form occurs in the idealized circumstance of homogeneous, isotropic turbulence decay. BHRZ discussed the form and its applications to spectral moments, showing that even with this simple idealization the standard singlepoint model could be derived. In their analysis, self-similarity means that the spectral form depends upon $k$ and $t$ through a function of a single combined variable $k L(t)$, in which $L(t)$ is a function with the dimensions of length (proportional to $k_{m}^{-1}$, where $k_{m}$ is the wave number at which $E_{\ell \ell}(k)$ is a maximum). In general, self-similarity means reduction of the number of independent variables by one, usually expressed by rescaling both spatial and wave-number vector components with functions of time as described in more detail below.

As a more complicated example, consider the spectral behavior of $E_{\ell \ell}(y, k, t)$ for a free shear layer. Note, first, that dimensional constraints for the overall (spectrally integrated) structure of the shear layer can be derived under the assumption that all aspects of initial conditions have been "forgotten." In the absence of viscosity, the incompressible behavior in a single fluid (Mach number $\rightarrow 0$ ) is characterized by a single dimensional number, the overall velocity difference across the layer, $U$. It then follows that the peak value of the turbulence energy per unit mass, $K_{0}$, equals a constant times $U^{2}$; also the width of the turbulence layer, $W$, varies in time as $U t$.

Again this description is an idealization. No free shear layer is absolutely flat across all space. More subtle, however, is the nonidealization associated with initial conditions, in particular with the structure of the spectrum as $k \rightarrow 0$. The effects of low-wave-number perturbations can persist forever and are therefore not "forgotten." The consequences are quite interesting and bear significantly on the issues of existence and uniqueness for self-similar forms, as discussed below. 


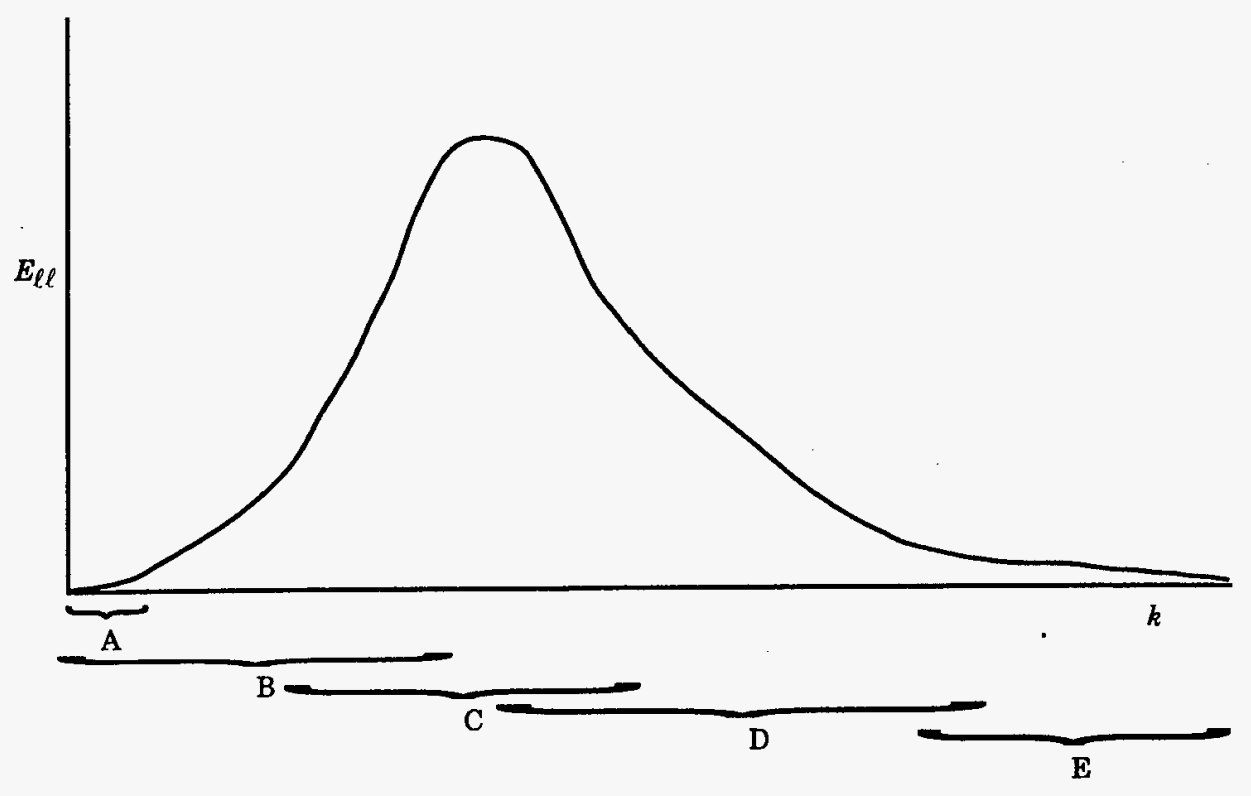

Fig. 1. Regions of spectral behavior in wave-number space.

For the example of a free shear layer, consider the behavior of $E_{\ell \ell}\left(o, k, t_{1}\right)$ at the midpoint of the layer at some particular time, $t$. Figure 1 shows the qualitative behavior of this quantity.

Interval $\mathrm{C}$ contains the dominant levels of turbulence, the total of which is essentially the same as the content of the entire spectrum. It is fed throughout region B by energyconserving coupling to the mean-flow gradient; i.e., the shear-stress component, $E_{x y}$, is removing energy from the mean flow at the same rate that turbulence energy is being created. This creation is of two types. One type is the thoroughly nonlinear coupling of turbulence structures that are comparable in scale to the width of the layer. The other type is the linear coupling (in the Kelvin-Helmholtz sense) to structures that are very large compared to $W$, which occurs in the interval $\mathrm{A}$.

A flux of turbulence energy from $\mathrm{C}$ to $\mathrm{A}$ also occurs as a result of inverse cascade, for which a dominant component occurs as a result of instability of the largest (strongly nonisotropic) "roll" structures. This instability takes energy from the mean-flow shear and draws the rolls together in pairs (the "vortex-pairing" process). Leith's (1990) stochastic backscatter also contributes to this inverse cascade. As discussed in Chapter III, the laminar-instability growth of turbulence in the interval A is also important but completely misrepresented by purely local descriptions. With the inclusion of nonlocal inertial effects, the $\mathrm{KH}$ source in A can be properly expressed. 
Thus the low-wave-number parts of the spectrum exhibit a very complicated set of interactions and couplings, the full richness of which can only be appreciated in consideration of the strong nonisotropy of the Reynolds stress components and of the structure as a function of the vector wave number $\mathbf{k}$. Questions of existence and uniqueness for self-similar forms can be answered only in the context of this full richness of processes. These questions are of three related types:

- What are the constraints on the low-wave-number parts of the spectrum that allow self-similarity?

- What is the nature of that self-similarity?

- Is there a concept of approximate self-similarity that is useful for a broad set of circumstances that do not admit exact self-similarity?

For higher wave numbers, the level of isotropy increases. The structural evolution is still governed by a variety of complicated nonlocal processes, as described in Chapters II and III. Interval C not only contains the dominant energy (and Reynolds stress components) but also is a transition interval. In $\mathrm{D}$, there is relatively little coupling to the mean flow; the Reynolds stress components move through $\mathbf{k}$ space to higher wave numbers in a manner that is neither creative nor destructive. This constant-flux inertial range is characterized by $k^{-5 / 3}$ behavior for $E_{\ell \ell}$ and by a somewhat more rapid decay of the deviator components of $E_{i j}$, which are destroyed both by cascade flux and the tendency for return to isotropy.

Finally, in the interval E, the effects of molecular viscosity become significant, and the Reynolds stress components decrease rapidly towards zero. In equilibrium the flux of turbulence energy through $\mathrm{D}$ equals the conversion rate to heat in $\mathrm{E}$.

True self-similarity means that at a later time the spectral variations of all the Reynolds stress components can be suitably scaled in $k$ and in magnitude so that the plots would look exactly the same. We can also describe self-symmetry in physical space by plotting $E_{\ell \ell}$ as a function of $y$ for a fixed value of $k$, or integrated over all values of $k$.

The central part, near $y=0$, receives energy by the various mechanisms of mean-flow coupling described above for low wave numbers. The lateral regions receive Reynolds stress components by turbulence self-diffusion and by the nonhomogeneous (nonlocal) contributions from the pressure-velocity correlations. The lateral regions sense the nonlocal effects of linear mean-flow coupling at low wave numbers, which decrease as $\exp (-2 k|y|)$. Because, to lowest order, turbulent self-diffusion is a nonlinear process, we might expect a rather sharp cut off at the edges of the shear layer, with relative low-amplitude wings extending beyond. 


\section{B. Self-Similar Time Extraction}

The scaling of coordinates ( $\mathbf{x}$ and $\mathbf{k}$ ) and magnitudes that define self-similarity can be expressed more precisely. Consider the behavior of $E(y, k, t)$, which has the dimensions of (distance $)^{3} /(\text { time })^{2}$. Suppose that we simultaneously stretch the space and time coordinates such that

$$
\begin{aligned}
& y \longrightarrow \omega^{n} y \\
& k \longrightarrow \omega^{-n} k \\
& t \longrightarrow \omega^{m} t,
\end{aligned}
$$

where $\omega$ is a dimensionless number that defines the degree of stretching, while $m$ and $n$ are numbers to be determined. We also stretch the magnitude of $E$ according to its dimensionality,

$$
E \longrightarrow \omega^{3 n-2 m} E .
$$

Our constraint of self-similarity in this process results in the equation

$$
\omega^{3 n-2 m} E(y, k, t)=E\left(\omega^{n} y, w^{-n} k, \omega^{m} t\right) .
$$

When we differentiate with respect to $\omega$ and then set $\omega=1$, the result is

$$
(3 n-2 m) E=n y \frac{\partial E}{\partial y}-n k \frac{\partial E}{\partial k}+m t \frac{\partial E}{\partial t},
$$

which can be solved to give

$$
E(y, k, t)=t^{\frac{3 n-2 m}{m}} F_{1}\left[\frac{y}{t^{n / m}}, k t^{n / m}\right],
$$

in which $F_{1}$ is an arbitrary function of its arguments. The origin of time is arbitrary, so that we may choose the onset of self-similarity at some time, $t_{0}$, and write

$$
\begin{aligned}
L(t) & \equiv L_{0}\left(t-t_{0}\right)^{n / m} \\
K(t) L(t) & \equiv K_{0} L_{0}\left(t-t_{0}\right)^{\frac{3 n-2 n}{m}},
\end{aligned}
$$

thus re-expressing the solution in the form

$$
E(y, k, t)=K(t) L(t) F\left[\frac{y}{L(t)}, k L(t)\right]
$$

with $F$ having absorbed some constants and likewise being an arbitrary function of its arguments. This expression describes the constraint of linear-stretching self-similarity on the form of $E$. It is derived without reference to the transport equation for $E$ and serves 
to distinguish from all possible transport solutions a particular subset with the property of self-similarity.

Applied to homogeneous, isotropic turbulence (independent of $y$ ), the result is conversion of a partial differential equation for $E(k, t)$ to an ordinary differential equation for $F[k L(t)]$. BHRZ discuss the properties of $F$ and obtain an explicit solution.

For the free shear-layer analysis in $k$ space the self-similarity constraint has likewise reduced the number of independent variables, but insertion into the transport equation nevertheless results in a partial differential equation for $F(y / L, k L)$, which has a much greater richness of possible solutions than the ordinary differential equation for homogeneous, isotropic circumstances. Already we see hints of the so-called "nonuniqueness of self-similar free-shear-layer turbulence."

The breadth of possibilities increases substantially when we examine the free shear layer in full vector, $\mathbf{k}$, space. Recognizing the dimensionality of $R_{i j}(\mathbf{x}, \mathbf{k}, t)$ as (distance $^{5} /(\text { time })^{2}$ we can derive the self-similar constraint in the form

$$
R_{i j}(\mathbf{x}, \mathbf{k}, t)=K(t) L^{3}(t) F_{i j}\left[\frac{\mathbf{x}}{L(t)}, \mathbf{k} L(t)\right],
$$

in which

$$
\begin{aligned}
L(t) & =L_{0}\left(t-t_{0}\right)^{n / m} \\
K(t) L^{3}(t) & =K_{0} L_{0}^{3}\left(t-t_{0}\right)^{\frac{5 n-2 m}{m}} .
\end{aligned}
$$

Insertion of this form into the full transport equation reduces the seven independent variables in the partial differential equation to six independent variables. For the free shear layer with physical-space dependence on $y$, only, the number of independent variables is four.

In this four-dimensional domain there are numerous possible dependencies of solutions on conditions at the boundaries. In particular, we believe that the structure of the turbulence near $|\mathbf{k}| \rightarrow 0$ is felt throughout the domain. There are various significant questions. How strongly is this influence propagated? Is it possible that most of the solutions cluster around a dominant form that shows relatively little effect from the conditions near $|\mathbf{k}|=0$ ? Certainly we can expect solutions to the full transport equation that do manifest strong dependence on conditions near $|\mathbf{k}|=0$ (and more generally, on initial conditions of various sorts), which are not included in the subset of self-similar solutions.

In homogeneous circumstances that are isotropic in $\mathbf{k}$ space, self-similarity is described by a single function of one independent variable, $k L$. The transport equations produce a single ordinary differential equation for this function, which is thus uniquely determined 
except for constants. More generally the self-similarity constraint results in a set of partial differential equations that describe nonunique families of form functions, which are capable of retaining information from the initial and boundary conditions from which the selfsimilarity evolved. Even in those circumstances that are not precisely self-similar, is it possible that there exists a meaningful concept of "almost self-similarity," in which we can identify broad classes of solutions that behave very closely to the truly self-similar solutions and exhibit relatively little influence on either the very large structures (of the mean flow) or the initial conditions? We postulate that the answer is yes. Moreover, we believe that the consequence is a meaningful validity to modeling with idealized self-similar solutions in which $K(t)$ and $L(t)$ are replaced by $K(\mathbf{x}, t)$ and $L(\mathbf{x}, t)$ so that the self-similarity is only local in both space and time. The implication is that meaningful modeling can be based on idealized self-similar forms even when the mean-flow circumstances change with position and time in ways that destroy precise self-similarity.

\section{A Free-Shear Example}

As a basis for exploring the implications of the above speculations, we examine in more detail the structure of the strictly self-similar forms arising in the presence of a free shear layer. One aspect of this structure seems especially remarkable. It is exhibited by the turbulence in an inviscid free-shear layer, described by the following set of five equations. We assume that the flow velocity satisfies $\mathbf{u}=u(y, t) \hat{\mathbf{x}}$. In the absence of viscosity and with purely local modeling, the equations describing the free-shear case can be written:

$$
\begin{aligned}
\frac{\partial E}{\partial t}-T(E) & =-2 u_{12} \tilde{E}_{12}+3 c_{F_{2}} u_{12} \frac{\partial\left(k \tilde{E}_{12}\right)}{\partial k} \\
\frac{\partial \tilde{E}_{+}}{\partial t}-T\left(\tilde{E}_{+}\right) & =\frac{1}{3} c_{+} u_{12} \tilde{E}_{12}+\frac{2}{3} c_{F} u_{12} \frac{\partial\left(k \tilde{E}_{12}\right)}{\partial k}-c_{M} k \sqrt{k E} \tilde{E}_{+} \\
\frac{\partial \tilde{E}_{-}}{\partial t}-T\left(\tilde{E}_{-}\right) & =-c_{-} u_{12} \tilde{E}_{12}-c_{M} k \sqrt{k E} \tilde{E}_{-}, \\
\frac{\partial \tilde{E}_{12}}{\partial t}-T\left(\tilde{E}_{12}\right) & =\frac{2}{15} u_{12} E+u_{12}\left(-c_{-} \tilde{E}_{-}+c_{+} \tilde{E}_{+}\right) \\
& +u_{12} \frac{\partial\left[k\left(2 c_{F} \tilde{E}_{+}-\frac{1}{30} E\right)\right]}{\partial k}-c_{M} k \sqrt{k E} \tilde{E}_{12} \\
\frac{\partial u_{12}(y, t)}{\partial t} & =-2 \frac{\partial^{2}}{\partial t^{2}} \int_{0}^{\infty} \tilde{E}_{12}(y, k, t) d k
\end{aligned}
$$

where $c_{ \pm} \equiv c_{B}-1 \pm c_{B_{1}}, E(y, k, t)$ equals the trace of the energy spectral tensor, $\tilde{E}_{ \pm}(y, k, t) \equiv\left(\tilde{E}_{22}(y, k, t) \pm \tilde{E}_{11}(y, k, t)\right) / 2, u_{12}(y, t) \equiv \partial u(y, t) / \partial y$, and where the tilde 
denotes the deviatoric, or anisotropic, part of the energy tensor. We have gleaned from numerical solutions of these equations a remarkable property when $T$ is taken to be the sum of two terms satisfying

$$
T(f) \equiv c_{D} \frac{\partial}{\partial x_{n}}\left(\nu_{T} \frac{\partial f}{\partial x_{n}}\right)+\frac{\partial}{\partial k}\left[\left(c_{2} k^{3} \frac{\partial}{\partial k}-c_{1} k^{2}\right) f\right]
$$

for an arbitrary function, $f(\mathbf{r}, k, t)$. Here $\nu_{T}(y, t) \equiv \int_{0}^{\infty} \frac{\sqrt{k E(y, k, t)}}{k^{2}} d k$. We have observed that self-similar, separable solutions appear to exist which have the structure,

$$
\begin{aligned}
E(y, t) & =K_{0} L(t) F(\eta) G(\omega g(\eta)), \\
E_{i j}(y, t) & =c_{i j} K_{0} L(t) F(\eta) G_{i j}(\omega g(\eta)),
\end{aligned}
$$

in which $\eta=y / L(t), \omega=k L(t), K_{0}$ is a constant specifying the energy density at $y=0$, $L(t)=L_{0}\left(1+t / t_{0}\right)$, where $L_{0}, t_{0}$, and the $\left\{c_{i j}\right\}$ are all constants, $F(0)=g(0)=1$, and where, finally, $\int_{0}^{\infty} G(\omega) d w=1$.

Using this variable-separated structure, one can verify that according to the final equation of the BHRZ model, the $\eta$-dependence of the shear, $u_{12}$, is merely proportional to $\partial(F / g) / \eta \partial \eta$. When one writes out each of the first four equations in terms of the variable-separated forms, one finds that each of them has terms involving $\omega$-derivatives of the $G$ functions multiplied by functions of $\eta$. These functions come in the following five varieties:

$$
\begin{aligned}
& 1-\eta \frac{F^{\prime}}{F}-N_{D} \alpha \sqrt{F g}\left[\frac{1}{2}\left(\frac{F^{\prime}}{F}\right)^{2}+\frac{1}{2} \frac{F^{\prime} g^{\prime}}{F g}+\frac{F^{\prime \prime}}{F}\right], \frac{1}{g} \sqrt{\frac{F}{g}} \\
& 1-\eta \frac{g^{\prime}}{g}-N_{D} \alpha \sqrt{F g}\left[\frac{5}{2} \frac{F^{\prime} g^{\prime}}{F g}+\frac{g^{\prime \prime}}{g}-\frac{1}{2}\left(\frac{g^{\prime}}{g}\right)^{2}\right], \frac{1}{\eta} \frac{d}{d \eta}\left(\frac{F}{g}\right) \\
& \sqrt{F g}\left(\frac{g^{\prime}}{g}\right)^{2}
\end{aligned}
$$

where there would be the possibility of some ambiguity if the last of the functions were not negligible. The primes denote differentiation with respect to the argument, which in this case is $\eta$. The constant $N_{D}$ is given by $c_{D} \int_{0}^{\infty} G^{\frac{1}{2}}(\omega) / \omega^{\frac{3}{2}} d \omega$, and the constant $\alpha$ by $L_{0} /\left(t_{0} \sqrt{K_{0}}\right)$. Since the last of the five functions has been shown by numerical computations to be negligible, we thus can arrive at nonlinear differential equations in the $G$ s alone if the remaining functions are proportional to each other (and so can be divided out). We find numerically that this is indeed the case, despite the fact that the proportionality requires three conditions to be placed upon only two functions, $F$ and $g$ ! 
To gain further insight into this separability of the energy and its deviatoric components into "form functions," $F(\eta), g(\eta)$, and $G_{i j}(\omega)$, we have replaced the diffusion term in $T$, the term prefaced by the constant, $c_{D}$, by a spatially conservative wavelike term prefaced by the constant $c_{W}$ so that

$$
T(f) \equiv c_{W} \frac{\partial}{\partial x_{n}}\left(\frac{\partial \nu_{T}}{\partial x_{n}} f\right)+\frac{\partial}{\partial k}\left[\left(c_{2} k^{3} \frac{\partial}{\partial k}-c_{1} k^{2}\right) f\right] .
$$

We were motivated to choose this model form for three reasons; namely (a) the numerical appearance of separability was anomalously highly sensitive to the value of $c_{D}$, (b) any term introduced should conserve energy in physical space, and (c) the presence of any term having the form, $\omega^{2} \frac{\partial^{2}}{\partial \omega^{2}}$, seemed to preclude the existence of exact separable solutions because they would overdetermine the $g$ and $F$ functions.

Without excluding the possibility that there may be other separable solutions in the presence of this wavelike term, we have found one separable solution of this equation that yields simple analytic forms for $g$ and $F$; namely $g(\eta)=\sqrt{1-\gamma \eta^{2} / 2}$ and $F(\eta)=g^{3}(\eta)$, where $N_{W} \alpha \gamma=-1$, and $N_{W}$ is defined analogously to $N_{D}$, where $c_{W}$ replaces $c_{D}$. In order that $F$ be spatially bounded, $\gamma$ must be positive. This implies then that $N_{W}$, or equivalently $c_{W}$ must be negative. One can verify then that the shear, $u_{12}(=\partial u / \partial y)$, is proportional to $\gamma$ and is thus spatially constant. (Of course, this solution does not satisfy the boundary conditions of a bounded shear flow layer.) In this case, one finds that the functions that multiply the $\omega$-derivatives of the $G$ functions that arise, corresponding to the functions listed above associated with the $T$ operator containing the diffusion terms, have the following values:

$$
\begin{aligned}
& 1-\eta \frac{F^{\prime}}{F}-\frac{N_{W} \alpha}{2} \sqrt{F g}\left[\frac{F^{\prime \prime}}{F}+\frac{g^{\prime \prime}}{g}+\frac{2 F^{\prime} g^{\prime}}{F g}+\frac{1}{2}\left(\frac{F^{\prime}}{F}\right)^{2}-\frac{1}{2}\left(\frac{g^{\prime}}{g}\right)^{2}\right]=2, \frac{1}{g} \sqrt{\frac{F}{g}}=1, \\
& 1-\eta \frac{g^{\prime}}{g}-\frac{N_{W} \alpha}{2} \sqrt{F g}\left[\frac{F^{\prime} g^{\prime}}{F g}+\left(\frac{g^{\prime}}{g}\right)^{2}\right]=1, \frac{1}{\eta} \frac{d}{d \eta}\left(\frac{F}{g}\right)=-\gamma .
\end{aligned}
$$

The fifth term in the earlier list is absent from this list because it resulted from a second derivative in $\omega$ arising from the diffusion term. There are no such second derivatives in the new wavelike term currently at hand.

The following equations are thereby produced determining the spectral distribution of the energy and its deviatoric components:

$$
\begin{aligned}
& 2 G(\omega)+\omega G^{\prime}(\omega)-\alpha c_{2} H(\omega)+\left(3 c_{F_{2}}-2\right) M \gamma c_{12}^{2} G_{12}(\omega)+3 c_{F_{2}} M \gamma c_{12}^{2} \omega G_{12}^{\prime}(\omega)=0, \\
& 2 G_{+}(\omega)+\omega G_{+}^{\prime}(\omega)-\alpha\left[c_{2} H_{+}(\omega)-c_{M} G^{\frac{1}{2}}(\omega) G_{+}(\omega)\right]+\frac{1}{3}\left(c_{+}+2\right) M \gamma c_{12}^{2} G_{12}(\omega)
\end{aligned}
$$




$$
\begin{aligned}
& \quad+\frac{2}{3} c_{F} M \gamma c_{12}^{2} \omega G_{12}^{\prime}(\omega)=0, \\
& 2 G_{-}(\omega)+\omega G_{-}^{\prime}(\omega)-\alpha\left[c_{2} H_{-}(\omega)-c_{M} G^{\frac{1}{2}}(\omega) G_{-}(\omega)\right]-c_{-} M \gamma c_{12}^{2} G_{12}(\omega)=0, \\
& 2 c_{12} G_{12}(\omega)+c_{12} \omega G_{12}^{\prime}(\omega)-\alpha\left[c_{2} H_{12}(\omega)-c_{M} G^{\frac{1}{2}}(\omega) G_{12}(\omega)\right] c_{12}-\frac{2}{15} M \gamma c_{12} G(\omega) \\
& \quad+M \gamma c_{12}\left[c_{-} G_{-}(\omega)+c_{+} G_{+}(\omega)\right]+M \gamma c_{12} \frac{d}{d \omega}\left\{\omega\left[2 c_{F} G_{+}(\omega)-\frac{1}{30} G(\omega)\right]\right\}=0,
\end{aligned}
$$

where we have employed the following definitions:

$$
\begin{aligned}
G_{+} & \equiv \frac{\left(c_{22} G_{22} \pm c_{11} G_{11}\right)}{2} \\
M & \equiv \int_{0}^{\infty} G_{12}(\omega) d \omega \\
\left(\begin{array}{c}
H(\omega) \\
H_{ \pm}(\omega) \\
H_{12}(\omega)
\end{array}\right) & \equiv \frac{d}{d \omega}\left\{G^{\frac{1}{2}}(\omega) \omega^{\frac{7}{2}+\frac{c_{1}}{c_{2}}} \frac{d}{d \omega}\left[\omega^{-\frac{c_{1}}{c_{2}}}\left(\begin{array}{c}
G(\omega) \\
G_{ \pm}(\omega) \\
G_{12}(\omega)
\end{array}\right)\right]\right\}
\end{aligned}
$$

These equations are merely a tensor generalization of BHRZ's Eq. (3.11).

That such a set of coupled nonlinear differential equations can lead to separable solutions is striking. However, the terms such as the diffusion term or the wavelike term are heuristically motivated. Indeed one would expect from a deeper analysis that the original forms of these terms require modifications, such as the following:

$$
\begin{gathered}
\frac{\partial}{\partial x_{n}}\left(\nu_{T} \frac{\partial E_{i j}}{\partial x_{n}}\right) \rightarrow \frac{\partial}{\partial x_{n}}\left(\nu_{T} \frac{\partial E_{i j}}{\partial x_{n}}+\nu_{T} \frac{\partial E_{n i}}{\partial x_{j}}+\nu_{T} \frac{\partial E_{j n}}{\partial x_{i}}\right), \\
\frac{\partial}{\partial x_{n}}\left(E_{i j} \frac{\partial \nu_{T}}{\partial x_{n}}\right) \rightarrow \frac{\partial}{\partial x_{n}}\left(E_{i j} \frac{\partial \nu_{T}}{\partial x_{n}}+E_{n i} \frac{\partial \nu_{T}}{\partial x_{j}}+E_{j n} \frac{\partial \nu_{T}}{\partial x_{i}}\right) .
\end{gathered}
$$

These modifications would be entered into the original BHRZ equations before the evolution equation for the traceless spectral energy tensor is derived. It may be too much to expect separable solutions for the traceless energy tensor to exist in the presence of these modifications! Indeed these kinds of modifications also can couple other tensor components of the spectral tensor into the equations that we have displayed above.

\section{A Simplified Subset Example}

To examine the nonuniqueness of self-similar solutions, consider a subset of the transport equation in the form

$$
\begin{aligned}
\frac{\partial R_{i j}}{\partial t} & =-R_{i \ell} \bar{\lambda}_{j \ell}-R_{j \ell} \bar{\lambda}_{i \ell} \\
& -c_{1} \frac{\partial}{\partial k_{\ell}}\left(k k_{\ell} \sqrt{k E} R_{i j}\right) \\
& +c_{m} k \sqrt{k E}\left(\frac{6 \pi^{2}}{k^{2}} \Delta_{i j} E_{\ell \ell}-R_{i j}\right)
\end{aligned}
$$


in which $\bar{\lambda}_{i j} \equiv \partial \bar{u}_{i} / \partial x_{j}$ and $\Delta_{i j} \equiv \delta_{i j}-\frac{k_{i} k_{j}}{k^{2}}$, and $E_{i j} \equiv \frac{1}{2} \int R_{i j} \frac{k^{2} d \Omega_{k}}{(2 \pi)^{3}}$. This form for the return-to-isotropy term ensures that angular integration in $\mathbf{k}$ space reduces that term to the simple form used by BHRZ. With a local rate in $k$ space for that term, it should be noted that integration over $k$ does not result in the single-point form for return to isotropy unless the variation of $E_{i j}$ follows a generic form as a function of $k$. The same observation can be made for angular integration in the transformation from $R_{i j}$ in $\mathbf{k}$ space to $E_{i j}$ in $k$ space for the alternative expression

$$
c_{m} k \sqrt{k E}\left(\frac{1}{2} \Delta_{i j} R_{\ell \ell}-R_{i j}\right),
$$

which contracts to zero. Because $R_{\ell \ell}$ is a function of $\mathbf{k}$, the angular integration over $\mathbf{k}$ space does not reduce to the BHRZ formulation unless $R_{i j}$ has an appropriate generic form such that $R_{n n}$ is a function of $k$, only.

We use this alternative expression for the return-to-isotropy term in the above transport equation to illustrate our ideas. Omitting any spatial transport terms enables us to concentrate on the behavior of the wave-number part of the self-similarity. $\bar{\lambda}_{i j}$ can refer either to the constant rate of homogeneous free shear or the local rate at the center of a free shear layer. Into this stripped-down transport equation we insert the self-similarity constraints,

$$
\begin{aligned}
R_{i j} & =K(t) L^{3}(t) F_{i j}[\mathbf{k} L(t)] \\
E & =K(t) L \tilde{F}[k L(t)] .
\end{aligned}
$$

Let $\boldsymbol{\xi} \equiv \mathbf{k} L(t)$, so that $\xi \equiv k L(t)$. The result is

$$
\begin{aligned}
\dot{K} L^{3} F_{i j} & +3 K L^{2} \dot{L} F_{i j}+K L^{2} \dot{L} \xi_{n} \frac{\partial F_{i j}}{\partial \xi_{n}} \\
& =-K L^{3}\left(F_{i \ell} \bar{\lambda}_{j \ell}+F_{\ell j} \bar{\lambda}_{i \ell}\right) \\
& -c_{1} L^{2} K \sqrt{K} \frac{\partial}{\partial \xi_{\ell}}\left[\xi \sqrt{\xi \tilde{F}(\xi)} \xi_{\ell} F_{i j}(\xi)\right] \\
& +c_{m} K L^{3 / 2} \tilde{F}^{\frac{1}{2}} \xi \sqrt{\xi}\left(\frac{1}{2} \Delta_{i j} F_{\ell \ell}-F_{i j}\right),
\end{aligned}
$$

in which $\Delta_{i j}(\mathbf{k}) \equiv \delta_{i j}-\frac{k_{i} k_{j}}{k^{2}}$. In order that this equation contain only the variable $\boldsymbol{\xi}$, it is necessary that there be no explicit dependence on $t$.

There are two possibilities. For homogeneous shear, $\bar{\lambda}_{i j}$ is constant in time and we require

and

$$
\begin{aligned}
& \frac{\dot{K}}{K} \equiv \text { constant } \equiv \alpha \\
& \frac{\dot{L}}{L} \equiv \text { constant } \equiv \beta
\end{aligned}
$$




$$
K L \sqrt{K} \equiv \text { constant. }
$$

Then

$$
\begin{aligned}
K & =K_{0} e^{\alpha t} \\
L & =L_{0} e^{\beta t}
\end{aligned}
$$

and

$$
\frac{3 \alpha}{2}+\beta=0 .
$$

Although these conditions remove time from the transport equation, which becomes an equation only in $\boldsymbol{\xi}$, there emerges a paradox. The exponential behavior of $K(t)$ and $L(t)$ contradicts the power-law behavior derived as part of the self-similar constraint. Thus it appears that a constant homogeneous shear is not amenable to self-similar description by this type of analysis. (Indeed, we believe that the idealization of such a mean-flow configuration is not attainable in nature.)

The second case, however, allows the extraction of consistent results. For the free shear, the only dimensionally allowable behavior for $\bar{\lambda}_{i j}$ is, as discussed above,

$$
\bar{\lambda}_{i j}=\lambda_{i j} U / L(t) .
$$

In this case the removal of explicit reference to time requires

$$
\begin{aligned}
\frac{\dot{K} L}{K} & \equiv \text { constant } \equiv \alpha \\
\dot{L} & \equiv \text { constant } \equiv \beta \\
K & \equiv \text { constant }
\end{aligned}
$$

Then self-similarity is described by

$$
\begin{aligned}
K & =\text { constant } \\
L & =\beta\left(t-t_{0}\right),
\end{aligned}
$$

which is, indeed, the correct physical behavior for a self-similar free shear layer.

The resulting equation for $F_{i j}$ becomes

$$
\begin{aligned}
(\alpha+3 \beta) F_{i j}+\beta \xi_{n} \frac{\partial F_{i j}}{\partial \xi_{n}} & =-U\left(F_{i \ell} \lambda_{j \ell}+F_{\ell j} \lambda_{i \ell}\right) \\
& -c_{1} \sqrt{K} \frac{\partial}{\partial \xi_{\ell}}\left[\xi \sqrt{\xi \tilde{F}(\xi)} \xi_{\ell} F_{i j}\right] \\
& +c_{m} \sqrt{K \tilde{F}} \xi\left(\frac{1}{2} \Delta_{i j} F_{\ell \ell}-F_{i j}\right) .
\end{aligned}
$$


Written in this form without the $c_{2}$ part of the cascade, the equation is amenable to characteristic analysis. With inclusion of the $c_{2}$ term, or more generally the nonlocal representations, the simple characteristic analysis is no longer possible. Such models do not preclude the self-similarity; they do, however, increase the analytical difficulty in extracting tractable self-similar form functions.

Note that

$$
\tilde{F}(\xi) \equiv \frac{1}{2} \int F_{\ell \ell}(\xi) \frac{\xi^{2} d \Omega_{\xi}}{(2 \pi)^{3}}
$$

so that characteristic analysis of even the "simple" equation, above, is challenging for the extraction of a solution.

To examine qualitatively the behavior, however, we consider the equation with $\tilde{F}(\xi)$ treated as constant. Let $c_{1}^{\prime} \equiv c_{1} \sqrt{K \tilde{F}}$ and $c_{m}^{\prime} \equiv c_{m} \sqrt{K \tilde{F}}$. Then the equation for $F_{i j}$ can be rewritten

$$
\begin{aligned}
\left(\beta+c_{1}^{\prime} \xi^{3 / 2}\right) \xi_{\ell} \frac{\partial F_{i j}}{\partial \xi_{\ell}} & =-U\left(F_{i \ell} \lambda_{j \ell}+F_{\ell j} \lambda_{i \ell}\right)-\left(\alpha+3 \beta+\frac{9}{2} c_{1}^{\prime} \xi^{3 / 2}\right) F_{i j} \\
& +c_{m}^{\prime} \xi\left(\frac{1}{2} F_{\ell \ell} \Delta_{i j}-F_{i j}\right) .
\end{aligned}
$$

Note that in this form of the transport equations, the directionality of characteristics is determined by

$$
\frac{d \xi_{1}}{\xi_{1}}=\frac{d \xi_{2}}{\xi_{2}}=\frac{d \xi_{3}}{\xi_{2}}
$$

so that $\xi_{1}=A \xi_{2}=B \xi_{2}$, with the geometry set by the constants, $A$ and $B$. Because $\xi^{2}=\xi_{1}^{2}+\xi_{2}^{2}=\xi_{3}^{2}$ along the characteristic, we note that

$$
\frac{d \xi_{1}}{\xi_{1}}=\frac{d \xi}{\xi}
$$

so that

$$
\frac{d F_{i j}}{d \xi}=\frac{-U\left(F_{i \ell} \lambda_{j \ell}+F_{\ell j} \lambda_{i \ell}\right)-\left(\alpha+3 \beta+\frac{9}{2} c_{1}^{\prime} \xi^{3 / 2}\right) F_{i j}+c_{m}^{\prime} \xi\left(\frac{1}{2} F_{\ell \ell} \Delta_{i j}-F_{i j}\right)}{\xi\left(\beta+c_{1}^{\prime} \xi^{3 / 2}\right)},
$$

with boundary conditions for $F_{i j}(0)$ that depend on $A$ and $B$.

Thus we have for this model an ordinary differential equation for the behavior of $F_{i j}(\xi)$, which we can examine for several interesting circumstances:

- the decay of homogeneous, nonisotropic turbulence, with $\lambda \equiv 0$, and

- the competition among shear, cascade, and the return to isotropy, with $\lambda \neq 0$.

Even with $\tilde{F}(\xi)$ not constant, a similar analysis can be performed leading to a nonlinear equation in which

$$
\tilde{F}(\xi)=\frac{1}{(2 \pi)^{2}} F_{\ell \ell}(\xi)
$$


If we include the $c_{2}$ term (diffusive in $\boldsymbol{\xi}$ space), other local terms that destroy the characteristic behavior, and/or various nonlocal modeling terms, this analysis then is no longer strictly valid; nevertheless many of the qualitative conclusions may still be relevant.

\section{E. Tensor Forms}

To carry through this type of analysis, it is reasonable to postulate that $F_{i j}$ can be written as a sum of available tensor forms that can describe the structure of the solution. Candidates for this purpose can be found among such forms as

$$
\lambda_{i j}, \quad \lambda_{i \ell} \lambda_{\ell j}, \quad \frac{\xi_{i} \xi_{j}}{\xi^{2}}, \quad \frac{\xi_{i} \xi_{\ell} \lambda_{j \ell}}{\xi^{2}}
$$

and similar dimensionless possibilities. The number of these tensor forms is fortunately bounded. Another class of possible forms comes from the initial conditions of $F_{i j}$. In the approach to self-similarity, the persistence of initial conditions varies with wave number. Qualitatively, we expect the memory to last for an "eddy turnover time," which is estimated for wave number $k$ to be $1 /(k \sqrt{k E})$. If $E(k)$ varies as $k^{n}$ near $k=0$, then the persistence time varies as $k^{-\left(\frac{n+3}{2}\right)}$, which is unbounded as $k \rightarrow 0$ for any reasonable value of $n$. This result suggests that $F_{i j}(0)$ is also a candidate for the construction of tensor forms and serves as a basis for our expectation that numerous different self-similar solutions exist, together with many others that are almost self-similar but not precisely so.

There are two types of time variations that are especially relevant to the consideration of self-similarity. One of them is concerned with the rate of approach to self-similarity following a perturbation to the mean-flow drive. The second serves as a tool for the identification of appropriate tensor forms.

Many authors have examined the tensor forms associated with specific mean-flow configurations (rapid-distortion theory). For homogeneous circumstances, spatial Fourier transformation is equivalent to spectral analysis in the two-point separation variable, and many interesting solutions are easily derived [Reynolds (1987)]: For our purposes, however, we proceed in a different manner, extracting the appropriate tensor forms through successive substitution of early-time solutions into the full (non-self-similar) equations.

For example, suppose that initially homogeneous, isotropic turbulence is sliced by a mean-flow shear. The lowest-order part of the non-self-similar solution is

$$
R_{i j}=R_{0}\left(\delta_{i j}-\frac{k_{i} k_{j}}{k^{2}}\right) .
$$

With suitable normalization of $\lambda_{i j}$ we can assume that $\lambda_{i j} \lambda_{i j} \equiv 1$. To first order in time, the tensor forms arising from this initial state come from the mean-flow coupling terms,

$$
\left(\delta_{i \ell}-\frac{k_{i} k_{\ell}}{k^{2}}\right) \lambda_{j \ell}+\left(\delta_{\ell j}-\frac{k_{\ell} k_{j}}{k^{2}}\right) \lambda_{i \ell}
$$


from which we extract

$$
\left(\lambda_{i j}+\lambda_{j i}\right)
$$

and

$$
\frac{k_{\ell}}{k^{2}}\left(k_{i} \lambda_{j \ell}+k_{j} \lambda_{i \ell}\right) .
$$

To second order in time we find, additionally,

$$
\left(\lambda_{i \ell}+\lambda_{\ell i}\right) \lambda_{j \ell}+\left(\lambda_{\ell j}+\lambda_{j \ell}\right) \lambda_{i \ell}
$$

and

$$
\frac{k_{n}}{k^{2}}\left(k_{i} \lambda_{\ell n}+k_{\ell} \lambda_{i n}\right) \lambda_{j \ell}+\frac{k_{n}}{k^{2}}\left(k_{\ell} \lambda_{j n}+k_{j} \lambda_{\ell n}\right) \lambda_{i \ell} .
$$

At this stage it is useful to recognize the relationships

$$
\begin{aligned}
\lambda_{i \ell} \lambda_{\ell j} & \equiv 0 \\
\lambda_{i \ell} \lambda_{n \ell} \lambda_{n j} & =\lambda_{i j} \\
\lambda_{\ell i} \lambda_{\ell n} \lambda_{m n} \lambda_{m j} & =\lambda_{\ell i} \lambda_{\ell j} \\
\lambda_{i \ell} \lambda_{n \ell} \lambda_{n m} \lambda_{j m} & =\lambda_{i \ell} \lambda_{j \ell} .
\end{aligned}
$$

Continuing the successive substitution and using the above relationships, we can obtain a closed set of independent tensor forms.

Note that from the nonhomogeneous spatial terms (e.g., turbulence self-diffusion) no additional tensor forms arise. For example,

$$
\begin{aligned}
\frac{\partial \lambda_{i j}}{\partial x_{n}} & =\frac{\partial \lambda_{i j}}{\partial \lambda} \frac{\partial \lambda}{\partial x_{n}}=\frac{\lambda_{i j}}{\lambda} \frac{\partial \lambda}{\partial x_{n}} \\
\frac{\partial \lambda_{i \ell} \lambda_{j \ell}}{\partial x_{n}} & =\frac{\lambda_{i \ell} \lambda_{j \ell}}{\lambda^{2}} \frac{\partial \lambda^{2}}{\partial x_{n}} .
\end{aligned}
$$

Thus, we can write

$$
F_{i j}(\boldsymbol{\xi})=\sum_{\sigma} A_{\sigma}[\text { Tensor Form }]_{\sigma},
$$

in which the $A_{\sigma}$ functions are scalar functions of the available scalar variables, such as $k$ and $k_{\ell} k_{n} \lambda_{\ell n}$.

Of greater interest, however, are various other initial conditions for $R_{i j}(t=0) \equiv$ $R_{i j}(0)$, from which a greater richness of tensor forms can emerge. With the simple returnto-isotropy model (the alternative form discussed above) in our equation for $R_{i j}$ we can examine

$$
\begin{aligned}
\frac{\partial R_{i j}}{\partial t} & =-R_{i \ell} \bar{\lambda}_{j \ell}-R_{\ell j} \bar{\lambda}_{i \ell} \\
& -c_{1} \frac{\partial}{\partial k_{\ell}}\left(k k_{\ell} \sqrt{k E} R_{i j}\right) \\
& +c_{\mu} k \sqrt{k E}\left(\frac{1}{2} R_{\ell \ell} \Delta_{i j}-R_{i j}\right) .
\end{aligned}
$$


The structure of $R_{i j}(0)$ potentially contains tensor forms having nothing to do with $\bar{\lambda}_{i j}$; they describe the effects of whatever stirring mechanism induced that initial state of turbulence. They can arise from the overall configuration of the experimental apparatus, for example the fan, screens, and walls of a wind tunnel. As a result of successive substitution into the equation for $R_{i j}$, we derive numerous hybrid tensor forms in addition to those that arise from an isotropic initial state or from the return-to-isotropy model, like

$$
k_{i} k_{\ell} R_{j \ell}(0), \quad \lambda_{i \ell} R_{j \ell}(0) .
$$

As noted above, the disappearance of initial conditions (here as a result of the return-toisotropy process) takes place progressively more slowly as $k \rightarrow 0$.

\section{F. Comments on Self-Similar Forms}

Although this type of successive substitution analysis enables the complete identification of independent tensor forms, it may contribute little else of value to the self-similar analysis. Introducing the tensor-form decomposition into the equation for $F_{i j}$ as a function of $\boldsymbol{\xi}$ and equating the coefficients of like tensors, we then obtain a set of coupled equations for the scalar functions, $A_{\sigma}$. The value of this approach, however, is more to demonstrate the existence of nonisotropic self-similar forms than to derive practical solutions like the form obtained by BHRZ for homogeneous, isotropic turbulence decay. A principal difficulty in working with the self-similar approach is that the equations for the $A_{\sigma}$ functions are eigenfunction equations. The solutions must be compatible with vanishing magnitudes for each $A_{\sigma}$ as $k \rightarrow 0$ and $k \rightarrow \infty$, with prescribed ratios among the functions in the first of these two limits. How, then, can we obtain self-similar solutions that are useful for our purposes (e.g., taking spectral moments for the derivation of single-point-nonspectralmodels)? Two techniques are much more tractable than brute-force attempts to extract eigenfunctions.

1. Numerical procedures for idealized circumstances, e.g., the idealization of an infinite free shear layer; relaxation from initial conditions through time to a self-similar state. (This technique can also be used for the self-similar eigenfunction equations through the introduction of a pseudo-time variable, but there does not seem to be any advantage to this approach.)

2. As an approximation, we can postulate the structure of the form functions and perform spectral moment integrations to reduce the problem to variations in $y$ and $t$ only. Even further, the postulate of form functions in $y$ reduces the analysis to variations in time only.

It should be noted that the close relationship between nonisotropy in physical space and that of $\mathbf{k}$ space is well demonstrated by the decomposition into tensor forms. This 
association is of much value in deriving angular averages of transport terms for the development of transport equations in $k$ space from those in $\mathbf{k}$ space.

Ordered, coherent structures emerge directly from this analysis. They are characterized by the tensor forms that dominate at low wave numbers. Spanwise rolls in a free shear layer furnish a prominent example. Closely related forms occur in variable-density turbulence transport [Steinkamp, Clark, and Harlow (1995)] where they are prominently manifested by large-scale fingers of interpenetration at the unstable interface between two fluids subjected to a normal pressure gradient. In both cases there is an associated continuous doubling process, vortex pairing for the shear layer, and double-doubling for the mixing layer.

In the variable-density mixing layer the coherent structures are strongly nonisotropic in both physical and $\mathbf{k}$ space. They may also be classified as representing an ordered (wavelike) part of the turbulence, in contrast to the isotropic part, which is disordered (diffusive). For constant-density turbulence there is a similar split into ordered and disordered parts. In physical space the propagation of turbulence can take place in essentially diffusive fashion; but as discussed in Chapter III, there can be an ordered (wave-like) component carried by nonlocal pressure waves, principally associated with coherent structures in nonhomogeneous configurations.

The most prominent external drivers for turbulence that are capable of imparting nonisotropic structure are

- free shear,

- boundary layer shear,

- rigid boundaries (even in the idealization of free slip),

- pressure gradients,

- pre-existing flow structured at very small wave numbers, and

- density gradients, including those that occur with constant density for each element of the fluid (material discontinuities, frozen-in entropy gradients with no molecular diffusion).

Each of these may vary in space and time, and in a stochastically steady flow the drive can vary along the mean-flow trajectories of fluid elements.

Competing effects occur in each facet of turbulence evolution. These are characterized especially in terms of rates, such as

- direct and inverse cascade in wave-number space, both local and nonlocal, 
- changes in drive in competition with the rate of approach to local selfsimilarity; maintenance of approximate self-similarity and/or generic form,

- equilibrium arising as a balance between nonisotropic drive and the return-to-isotropy processes,

- critical Reynolds number for turbulence transition as a competition between instability and viscous dissipation to heat, and

- uniqueness of self-similarity as a competition between long-wave-length instability growth and inverse cascade driven by mean flow gradients or second-point effects. 


\section{TURBULENCE SELF-DIFFUSION}

\section{A. Introduction}

Two complementary roles for the actions of the triple correlations have been suggested. First, the triple correlations lead to a transfer of turbulence energy from large scales to small scales (the turbulence "cascade") and second, in inhomogeneous flows, the triple correlations contribute to the self-propagation of turbulence from regions of high intensity to regions of low intensity (turbulence "self-diffusion"). In this section we will restrict our attention to the physical-space effects of the triple-correlations and the pressure-velocity correlations for inhomogeneous circumstances. In particular, we will examine the so-called "turbulence self-diffusion" phenomenon using three tools:

(1) the moment equations,

(2) direct numerical simulations of the Navier-Stokes equations, and

(3) turbulence models.

We will use the moment equations as a guide in modeling the triple correlations and to motivate the direct numerical simulations. The direct numerical simulations will be used to determine the plausibility of various potential models, and the turbulence models will be used to demonstrate the consequence of the modeling assumptions at high Reynolds numbers.

\section{B. The Navier-Stokes Moment Equations}

We begin with the Navier-Stokes equations for an incompressible fluid of constant density $\rho$,

$$
\frac{\partial u_{i}}{\partial t}+\frac{\partial u_{i} u_{n}}{\partial x_{n}}=-\frac{1}{\rho} \frac{\partial p}{\partial x_{i}}+\nu \frac{\partial^{2} u_{i}}{\partial x_{n} \partial x_{n}}
$$

with the condition that the velocity field will be divergence free;

$$
\frac{\partial u_{n}}{\partial x_{n}}=0
$$

We next decompose the variables into mean and fluctuating parts (the Reynolds decomposition), $u_{i}=U_{i}+u_{i}^{\prime}$ and $p / \rho=P+p^{\prime}$ and average, yielding the mean flow equation,

$$
\frac{D U_{i}}{D t}=-\frac{\partial R_{i n}}{\partial x_{n}}-\frac{\partial P}{\partial x_{i}}+\nu \frac{\partial^{2} U_{i}}{\partial x_{n} \partial x_{n}},
$$

where the term on the left-hand side is a "average" material derivative, e.g.,

$$
\frac{D \phi}{D t}=\frac{\partial \phi}{\partial t}+U_{n} \frac{\partial \phi}{\partial x_{n}}
$$


$R_{i j}$ is, of course, the Reynolds stress tensor, $R_{i j}=\overline{u_{i}^{\prime} u_{j}^{\prime}}$. We derive the equation for the fluctuation by subtracting the averaged equation from the instantaneous equation. The instantaneous fluctuating velocity is

$$
\frac{D u_{i}^{\prime}}{D t}=-\frac{\partial u_{n}^{\prime} U_{i}}{\partial x_{n}}+\frac{\partial}{\partial x_{n}}\left(R_{i n}-u_{n}^{\prime} u_{i}^{\prime}\right)-\frac{\partial p^{\prime}}{\partial x_{i}}+\nu \frac{\partial^{2} u_{i}^{\prime}}{\partial x_{n} \partial x_{n}} .
$$

Using this equation we can derive the Reynolds stress equation by constructing the equation for $u_{i}^{\prime} u_{j}^{\prime}$,

$$
\begin{aligned}
\frac{D u_{i}^{\prime} u_{j}^{\prime}}{D t}= & -\left(u_{j}^{\prime} u_{n}^{\prime} \frac{\partial U_{i}}{\partial x_{n}}+u_{i}^{\prime} u_{n}^{\prime} \frac{\partial U_{j}}{\partial x_{n}}\right) \\
& +\frac{\partial}{\partial x_{n}}\left(R_{i n} u_{j}^{\prime}+R_{j n} u_{i}^{\prime}-u_{i}^{\prime} u_{j}^{\prime} u_{n}^{\prime}\right) \\
& -\left(R_{i n} \frac{\partial u_{j}^{\prime}}{\partial x_{n}}+R_{j n} \frac{\partial u_{i}^{\prime}}{\partial x_{n}}\right) \\
& -\frac{\partial}{\partial x_{n}}\left(p^{\prime} u_{i} \delta_{j n}+p^{\prime} u_{j}^{\prime} \delta_{i n}\right)+p^{\prime} \frac{\partial}{\partial x_{n}}\left(u_{i}^{\prime} \delta_{j n}+u_{i} \delta_{j n}\right) \\
& +\nu \frac{\partial^{2} u_{i}^{\prime} u_{j}^{\prime}}{\partial x_{n} \partial x_{n}}-2 \nu \frac{\partial u_{i}^{\prime}}{\partial x_{n}} \frac{\partial u_{j}^{\prime}}{\partial x_{n}},
\end{aligned}
$$

and averaging

$$
\begin{aligned}
\frac{D R_{i j}}{D t}= & -\left(R_{j n} \frac{\partial U_{i}}{\partial x_{n}}+R_{i n} \frac{\partial U_{j}}{\partial x_{n}}\right) \\
& -\frac{\partial}{\partial x_{n}}\left(R_{i j n}+\overline{p^{\prime} u_{i}^{\prime}} \delta_{j n}+\overline{p^{\prime} u_{j}^{\prime}} \delta_{i n}\right) \\
& +\overline{p^{\prime} \frac{\partial}{\partial x_{n}}\left(u_{i}^{\prime} \delta_{j n}+u_{i}^{\prime} \delta_{j n}\right)} \\
& +\nu \frac{\partial^{2} R_{i j}}{\partial x_{n} \partial x_{n}}-2 \epsilon_{i j},
\end{aligned}
$$

where $\epsilon_{i j}$ is the dissipation tensor,

$$
\epsilon_{i j}=\nu \overline{\frac{\partial u_{i}^{\prime}}{\partial x_{n}} \frac{\partial u_{j}^{\prime}}{\partial x_{n}}}
$$

and $R_{i j k}$ is the third moment of the fluctuating velocities, $R_{i j k}=\overline{u_{i}^{\prime} u_{j}^{\prime} u_{k}^{\prime}}$. Note that this tensor appears in the equation in a fully conservative fashion and is symmetric on all three 
[9

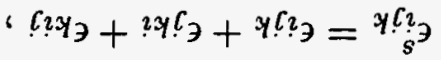

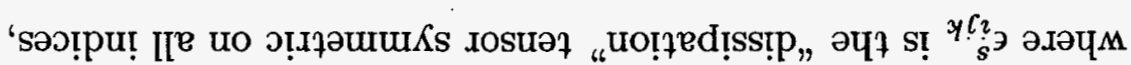

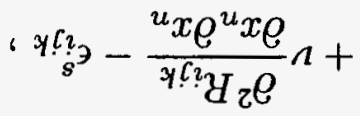

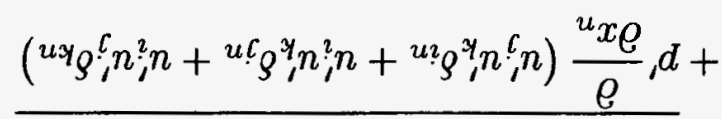

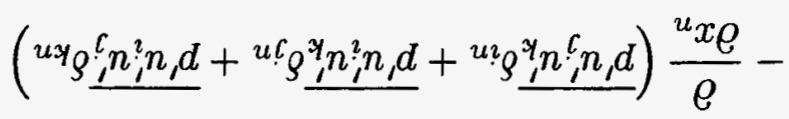

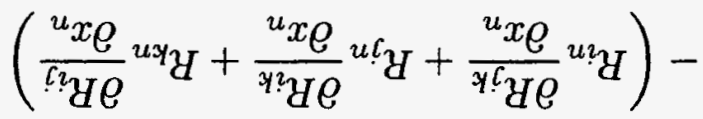

$$
\begin{aligned}
& \left({ }^{u y} c^{c_{2}} y-{ }^{c_{\imath}} y{ }^{u y} y+{ }^{y ?} y{ }^{u c} y+{ }^{y} c_{y} y^{u ?} y\right) \frac{{ }^{u} x \varrho}{\varrho}+
\end{aligned}
$$

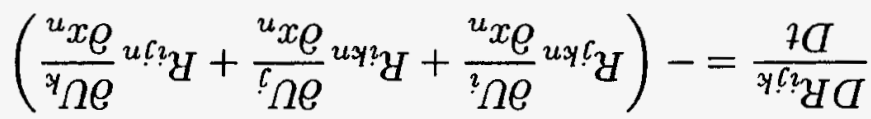

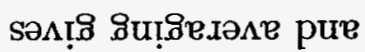

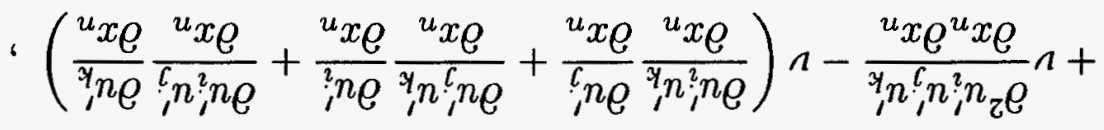

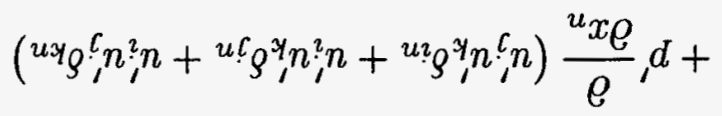

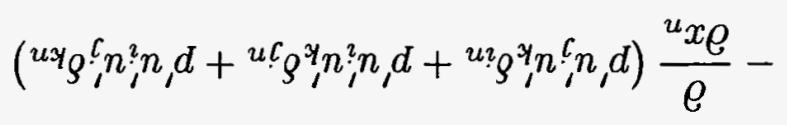

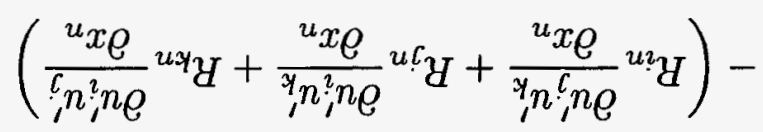

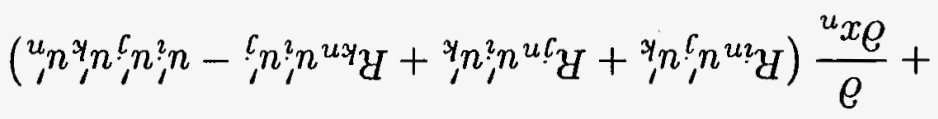

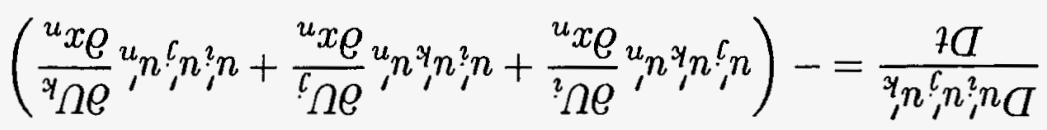

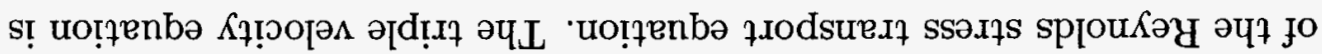

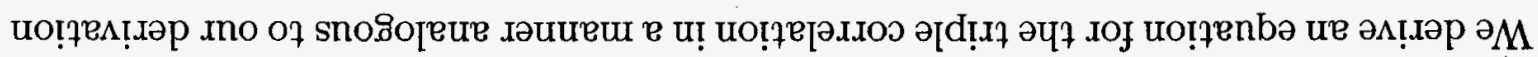

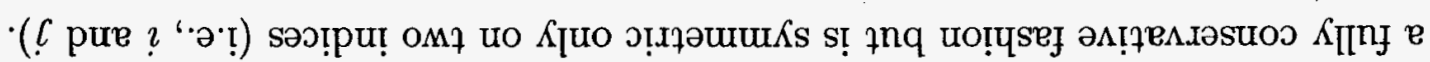

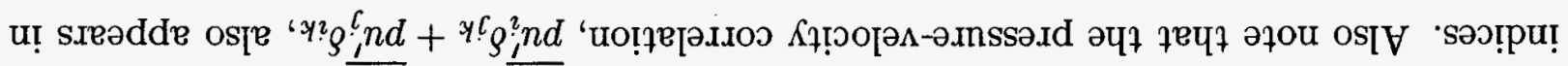


and

$$
\epsilon_{i j k}=\nu \overline{\frac{\partial u_{i}^{\prime} u_{j}^{\prime}}{\partial x_{n}} \frac{\partial u_{k}^{\prime}}{\partial x_{n}}} .
$$

In addition to these equations, the divergence-free character of the velocity field can be exploited to derive a Poisson equation for pressure divided by density, for the average pressure/density,

$$
-\frac{\partial^{2} P}{\partial x_{n} \partial x_{n}}=\frac{\partial^{2}}{\partial x_{i} \partial x_{j}}\left(U_{i} U_{j}+R_{i j}\right),
$$

and for the fluctuating pressure/density,

$$
-\frac{\partial^{2} p^{\prime}}{\partial x_{n} \partial x_{n}}=\frac{\partial^{2}}{\partial x_{i} \partial x_{j}}\left(u_{i}^{\prime} u_{j}^{\prime}-R_{i j}+2 U_{i} u_{j}^{\prime}\right) .
$$

\section{The Pressure-Velocity Correlation}

The pressure equations can be inverted (e.g., via a Green's function approach) to yield an integro-differential expression relating the pressure at a point in space to the velocity field (mean and fluctuating) over the entire domain. This expression can then be used to derive expressions for the pressure-velocity and pressure-strain correlations. These relations are highly opaque, involving three-dimensional integrals of two-point correlations over the given domain. If one restricts ones attention to homogeneous turbulence and pursues a modeling in Fourier space, some simplifications arise. In physical space (as opposed to Fourier space) the intrinsic three-dimensionality and integral nature remain and can only be "eliminated" by making relatively bold assertions (either explicitly or implicitly) regarding the nonlocal nature of these processes. For the present analysis, we are concerned with features intrinsic to inhomogeneous turbulence, and very little rigorous analysis can be performed to produce tractable differential closures for these pressure terms in physical space.

We will presently argue that the conservative pressure-velocity correlations serve as a minor inhibition to the process of turbulence self-propagation and discuss how such an effect might be represented in a differential closure. However, we do not claim that such a representation can possess adequate physical fidelity to warrant their inclusion in a differential closure. It seems possible that under some circumstances, errors caused by representing this "nonlocal" effect as a purely local effect are, in fact, greater than errors caused by neglecting the effects of pressure-velocity correlations entirely. This shouldn't be considered as a condemnation of current engineering dosures. In fact, many of the engineering closures that neglect these effects have been shown to yield good results under a variety of circumstances, thus suggesting that neglecting the effects of $\overline{p^{\prime} u_{i}^{\prime}}$ is 
appropriate in many circumstances. Rather, we merely suggest that one might profitably consider "nonlocal" or integral approaches to modeling the effects of $\overline{p^{\prime} u_{i}^{\prime}}$ when attempting to increase the regime of the validity of these closures.

We begin by examining the Poisson equation for the fluctuating pressure/density for the case of an inhomogeneous, undriven turbulent field (this is also the circumstance we will simulate). The equation is

$$
-\frac{\partial^{2} p^{\prime}}{\partial x_{n} \partial x_{n}}=\frac{\partial^{2}}{\partial x_{i} \partial x_{j}}\left(u_{i}^{\prime} u_{j}^{\prime}-R_{i j}\right) .
$$

Now letting $r_{i j}=\left(u_{i}^{\prime} u_{j}^{\prime}-R_{i j}\right)$ and decomposing this tensor into its trace and deviator (denoted by the superscript "d"),

$$
-\frac{\partial^{2} p^{\prime}}{\partial x_{n} \partial x_{n}}=\frac{\partial^{2}}{\partial x_{i} \partial x_{j}}\left(\frac{1}{3} \delta_{i j} r_{m m}+r_{i j}^{d}\right)
$$

or

$$
-\frac{\partial^{2} p^{\prime}}{\partial x_{n} \partial x_{n}}=\frac{1}{3} \frac{\partial^{2} r_{m m}}{\partial x_{n} \partial x_{n}}+\frac{\partial^{2} r_{i j}^{d}}{\partial x_{i} \partial x_{j}} .
$$

These equations suggest that for weakly anisotropic turbulence in the absence of mean-flow drive or wall effects,

$$
-p^{\prime} \approx \frac{1}{3} r_{m m}=\frac{1}{3}\left(u_{m} u_{m}-R_{m m}\right)
$$

A possible form for the pressure-velocity correlation in weakly anisotropic turbulence might be

$$
-\overline{p^{\prime} u_{i}^{\prime}}=\alpha_{p} \frac{1}{3}\left(\overline{u_{m}^{\prime} u_{m}^{\prime} u_{i}^{\prime}-R_{m m} u_{i}^{\prime}}\right)=\alpha_{p} \frac{1}{3} \overline{u_{m}^{\prime} u_{m}^{\prime} u_{i}^{\prime}}=\frac{\alpha_{p}}{3} R_{m m i},
$$

where $\alpha_{p}$ is a dimensionless coefficient. The direct numerical simulations appear to give $\alpha_{p} \approx 0.33$. (The details of the simulations will be discussed later.) Thus a "complete" differential closure for the conservative moments in physical space in the Reynolds stress transport equation might be

$$
\begin{aligned}
-D_{i j k} & =R_{i j k}+\overline{p^{\prime} u_{i}^{\prime}} \delta_{j k}+\overline{p^{\prime} u_{j}^{\prime}} \delta_{i k} \\
& \approx R_{i j k}-\frac{\alpha_{p}}{3}\left(R_{n n i} \delta_{j k}+R_{n n j} \delta_{i k}\right) .
\end{aligned}
$$

The pressure-velocity correlation appears to counteract the effects of the triple correlation. Thus if the velocities "align" to produce something like a net flux of " $R_{i j}$ " the pressure-velocity correlations mitigate this effect in a manner perhaps analogous to a 
body drag. This mitigation is somewhat more apparent if we decompose the $R_{i j k}$-tensor into a traceless part ("deviator") and its traces,

$$
R_{i j k}=R_{i j k}^{d}+\frac{1}{5}\left(\delta_{i j} R_{n n k}+\delta_{i k} R_{n n j}+\delta_{j k} R_{n n i}\right) .
$$

Substitution into $D_{i j k}$ gives

$$
-D_{i j k}=R_{i j k}^{d}+\frac{1}{5}\left[\delta_{i j} R_{n n k}+\left(1-\frac{5}{3} \alpha_{p}\right)\left(\delta_{i k} R_{n n j}+\delta_{j k} R_{n n i}\right)\right] .
$$

Now recall that the Reynolds stress equation contains derivatives on the " $k$ " index. Consider elements of $-D_{\beta \beta k}$ (with no summation on Greek indices),

$$
-D_{\beta \beta k}=R_{\beta \beta k}^{d}+\frac{1}{5}\left[\delta_{\beta \beta} R_{n n k}+\left(1-\frac{5}{3} \alpha_{p}\right)\left(\delta_{\beta k} R_{n n \beta}+\delta_{\beta k} R_{n n \beta}\right)\right] .
$$

If $\beta=k$, then the velocities in the triple correlation are the same component (i.e., the fluctuating velocities are aligned with the direction in which the derivative will be taken), and the effects of the pressure-velocity correlations are felt via $\alpha_{p}$,

$$
-D_{\beta \beta \beta}=R_{\beta \beta \beta}^{d}+\frac{1}{5}\left(3-\frac{10}{3} \alpha_{p}\right) R_{n n \beta} .
$$

On the other hand, consider $i=j=\gamma \neq \beta$, then the effects of the pressure-velocity correlation are not felt,

$$
-D_{\gamma \gamma \beta}=R_{\gamma \gamma \beta}^{d}+\frac{1}{5}\left[\delta_{\gamma \gamma} R_{n n \beta}\right] .
$$

Thus the pressure-velocity correlations appear to produce a preferential inhibition to turbulence self-diffusion. Note that if $\alpha_{p}=3 / 5$ the effect of $-D_{i j k}$ would show up as an essentially isotropic effect (except, of course, for possible contributions from the deviator $R_{i j k}^{d}$ ). As stated above, the direct numerical simulations suggest a value of $\alpha_{p} \approx 0.33$. This produces a correction of approximately 33 percent (neglecting the deviator) in the diffusion term $-D_{\beta \beta \beta}$. To complete this proposal for modeling of the conservative correlations, we must produce an algebraic closure for the triple correlations.

\section{The Triple Correlation Equation}

Next, we will attempt to provide an algebraic closure for the third-order moment of velocity, $R_{i j k}$. Note that one could close the model at the fourth-order level and develop a closed equation for the third-order moment-we have not yet pursued this option. Several rationalizations can be constructed for closing the equations at the second-order: 
First, if the field is not "too far" from Gaussian (whatever "too far" means), then a quasi-normal assumption might be invoked to close the equations at the third-order. If the turbulence is not "too far" from equilibrium, then the triple correlations may be represented algebraically as an "equilibrium solution" of some sort. These are essentially the assumptions of the "EDQNM" model.

Second, if the moments converge in some sense, then we might hope that the bulk of the physical effects can be expressed in the dynamics of the second-order correlation and the third-order (and higher) correlations might be algebraic corrections to the second-order correlations.

Third (a purely practical consideration), it is not clear that for many applications the additional computational complexity of a higher-order closure are warranted. The additional phenomenological assumptions required to close the higher order equations may nullify any benefits accrued by maintaining higher order correlations. In addition, many of the difficulties associated with turbulence modeling are not directly related to the closure of the hierarchy of equations. Rather, they are due to difficulties in treating the fully three-dimensional integro-differential terms arising from the pressure correlations, and these difficulties are present at any given order of closure. Thus a higher-order closure which invokes a local expression for pressure correlations is probably not an improvement over a lower-order closure, since the damage to physical fidelity is probably made at the level of the approximation for the pressure terms.

With these concerns in mind, we will invoke both an equilibrium assumption and a quasi-normal assumption to examine the triple correlation. First, we will assume that production approximately balances dissipation effects (i.e., we assume we are near "equilibrium") and that the net effect of the terms analogous to pressure-strain correlations are essentially production terms. Neglecting viscous diffusion we have

$$
\begin{aligned}
\frac{D R_{i j k}}{D t} & =\frac{\partial}{\partial x_{n}}\left(R_{i n} R_{j k}+R_{j n} R_{i k}+R_{k n} R_{i j}-R_{i j k n}\right) \\
& -R_{i n} \frac{\partial R_{j k}}{\partial x_{n}}-R_{j n} \frac{\partial R_{i k}}{\partial x_{n}}-R_{k n} \frac{\partial R_{i j}}{\partial x_{n}} \\
& -\frac{\partial}{\partial x_{n}}\left(\overline{p^{\prime} u_{j}^{\prime} u_{k}^{\prime}} \delta_{i n}+\overline{p^{\prime} u_{k}^{\prime} u_{i}^{\prime}} \delta_{j n}+\overline{p^{\prime} u_{i}^{\prime} u_{j}^{\prime}} \delta_{k n}\right) .
\end{aligned}
$$

Next, substitute the previous approximation for the fluctuating pressure into the $R_{i j k^{-}}$ equation,

$$
\frac{D R_{i j k}}{D t}=\frac{\partial}{\partial x_{n}}\left(R_{i n} R_{j k}+R_{j n} R_{i k}+R_{k n} R_{i j}-R_{i j k n}\right)
$$




$$
\begin{aligned}
& -R_{i n} \frac{\partial R_{j k}}{\partial x_{n}}-R_{j n} \frac{\partial R_{i k}}{\partial x_{n}}-R_{k n} \frac{\partial R_{i j}}{\partial x_{n}} \\
& +\frac{\alpha_{p}}{3} \frac{\partial}{\partial x_{n}}\left(R_{m m j k} \delta_{i n}+R_{m m i k} \delta_{j n}+R_{m m i j} \delta_{k n}\right) \\
& -\frac{\alpha_{p}}{3} \frac{\partial}{\partial x_{n}}\left(R_{m m} R_{j k} \delta_{i n}+R_{m m} R_{i k} \delta_{j n}+R_{m m} R_{i j} \delta_{k n}\right) .
\end{aligned}
$$

Next we invoke a "quasi-normal" approximation in physical space, i.e.,

$$
R_{i j k n}=R_{i j} R_{k n}+R_{i k} R_{j n}+R_{i n} R_{k j}
$$

and a Markovian-like assumption, i.e.,

$$
\frac{D R_{i j k}}{D t} \approx \frac{R_{i j k}}{\tau}
$$

where $\tau$ is a turbulent time scale, e.g.,

$$
\tau=C_{T} \frac{R_{n n}}{2 \epsilon} .
$$

The following "algebraic" closure for the triple correlation might be suggested:

$$
\begin{aligned}
\frac{R_{i j k}}{\tau}= & -\left(R_{i n} \frac{\partial R_{j k}}{\partial x_{n}}+R_{j n} \frac{\partial R_{i k}}{\partial x_{n}}+R_{k n} \frac{\partial R_{i j}}{\partial x_{n}}\right) \\
& +\alpha_{p} \frac{2}{3} \frac{\partial}{\partial x_{n}}\left(R_{j m} R_{m k} \delta_{i n}+R_{i m} R_{m k} \delta_{j n}+R_{i m} R_{m j} \delta_{k n}\right) .
\end{aligned}
$$

This representation is fully symmetric on the indices $i j k$. Substituting this term into the equation for the turbulence self-diffusion tensor $D_{i j k}$ gives

$$
\begin{aligned}
\frac{D_{i j k}}{\tau} & =R_{i n} \frac{\partial R_{j k}}{\partial x_{n}}+R_{j n} \frac{\partial R_{i k}}{\partial x_{n}}+R_{k n} \frac{\partial R_{i j}}{\partial x_{n}} \\
& -\alpha_{p} \frac{2}{3} \frac{\partial}{\partial x_{n}}\left(R_{j m} R_{m k} \delta_{i n}+R_{i m} R_{m k} \delta_{j n}+R_{i m} R_{m j} \delta_{k n}\right) \\
& -\frac{\alpha_{p}}{3}\left(\delta_{i l} \delta_{j k}+\delta_{j l} \delta_{i k}\right)\left[R_{l n} \frac{\partial R_{m m}}{\partial x_{n}}+2 R_{m n} \frac{\partial R_{l m}}{\partial x_{n}}\right] \\
& +2\left(\frac{\alpha_{p}}{3}\right)^{2}\left(\delta_{i l} \delta_{j k}+\delta_{j l} \delta_{i k}\right) \frac{\partial}{\partial x_{n}}\left(R_{p m} R_{m p} \delta_{l n}+2 R_{l m} R_{m n}\right)
\end{aligned}
$$


or, in a somewhat more verbose form,

$$
\begin{aligned}
\frac{\partial D_{i j k}}{\partial x_{k}} & =\frac{\partial}{\partial x_{k}}\left[\tau\left(R_{i n} \frac{\partial R_{j k}}{\partial x_{n}}+R_{j n} \frac{\partial R_{i k}}{\partial x_{n}}+R_{k n} \frac{\partial R_{i j}}{\partial x_{n}}\right)\right] \\
& -\frac{\alpha_{p}}{3} \frac{\partial}{\partial x_{j}}\left[\tau\left(R_{i n} \frac{\partial R_{m m}}{\partial x_{n}}+2 R_{m n} \frac{\partial R_{i m}}{\partial x_{n}}\right)\right] \\
& -\frac{\alpha_{p}}{3} \frac{\partial}{\partial x_{i}}\left[\tau\left(R_{j n} \frac{\partial R_{m m}}{\partial x_{n}}+2 R_{m n} \frac{\partial R_{j m}}{\partial x_{n}}\right)\right] \\
& -2 \frac{\alpha_{p}}{3} \frac{\partial}{\partial x_{k}}\left[\tau\left(\frac{\partial R_{j m} R_{m k}}{\partial x_{i}}+\frac{\partial R_{i m} R_{m k}}{\partial x_{j}}+\frac{\partial R_{i m} R_{m j}}{\partial x_{k}}\right)\right] \\
& +2\left(\frac{\alpha_{p}}{3}\right)^{2} \frac{\partial}{\partial x_{j}}\left[\tau\left(\frac{\partial R_{p m} R_{m p}}{\partial x_{i}}+2 \frac{\partial R_{i m} R_{m n}}{\partial x_{n}}\right)\right] \\
& +2\left(\frac{\alpha_{p}}{3}\right)^{2} \frac{\partial}{\partial x_{i}}\left[\tau\left(\frac{\partial R_{p m} R_{m p}}{\partial x_{j}}+2 \frac{\partial R_{j m} R_{m n}}{\partial x_{n}}\right)\right]
\end{aligned}
$$

Note that this model appears to be analogous to a nonlinear diffusion of Reynolds stress. Because we have satisfied the symmetry condition for the triple-velocity correlation, this model will reduce to the essentially isotropic form discussed above for the case of $\alpha_{p}=3 / 5$. Note also that the form proposed by Daly and Harlow (1970) corresponds, in essence, to the last term in parenthesis in the first line. The fully symmetric form proposed by Hanjalic̀ and Launder (1972) [and also suggested by Besnard, Harlow, Rauenzahn and Zemach $(1992,1996)]$ corresponds to the first line. Of course, the terms multiplied by $\alpha_{p}$ represent corrections to the correlation due to the presence of the conservative pressurevelocity correlations. We expect that the order of importance of the various terms is as indicated by their order in the equation. Clearly, for small $\alpha_{p}$, the terms that are linear and quadratic in $\alpha_{p}$ (the pressure-correction represented in the $D_{i j k}$ acting on the pressure-correction acting on $R_{i j k}$ ) are much smaller than the other terms and may be negligible compared to the size of other errors in our approximation. Thus the most obvious simplification would be to simply neglect the effects of the pressure (i.e., set $\alpha_{p}=0$ ), thus yielding the Hanjalic and Launder (1972) form;

$$
\frac{\partial D_{i j k}}{\partial x_{k}}=\frac{\partial}{\partial x_{k}}\left[\tau\left(R_{i n} \frac{\partial R_{j k}}{\partial x_{n}}+R_{j n} \frac{\partial R_{i k}}{\partial x_{n}}+R_{k n} \frac{\partial R_{i j}}{\partial x_{n}}\right)\right] .
$$

Now consider an element $D_{\beta \beta k}$ (again, no summation on Greek indices):

$$
\frac{\partial D_{\beta \beta k}}{\partial x_{k}}=\frac{\partial}{\partial x_{k}}\left[\tau\left(2 R_{\beta n} \frac{\partial R_{\beta k}}{\partial x_{n}}+R_{k n} \frac{\partial R_{\beta \beta}}{\partial x_{n}}\right)\right] .
$$


We will first consider the case wherein the gradients are in the $k=\beta$ direction. We have

$$
\frac{\partial D_{\beta \beta \beta}}{\partial x_{\beta}}=3 \frac{\partial}{\partial x_{\beta}}\left[\tau\left(R_{\beta \beta} \frac{\partial R_{\beta \beta}}{\partial x_{\beta}}\right)\right] .
$$

Next, consider the case where $i=j=\gamma \neq \beta$. This gives

$$
\begin{aligned}
\frac{\partial D_{\gamma \gamma \beta}}{\partial x_{\beta}} & =\frac{\partial}{\partial x_{\beta}}\left[\tau\left(2 R_{\beta \gamma} \frac{\partial R_{\beta \gamma}}{\partial x_{\beta}}+R_{\beta \beta} \frac{\partial R_{\gamma \gamma}}{\partial x_{\beta}}\right)\right] \\
& =\frac{\partial}{\partial x_{\beta}}\left[\tau\left(\frac{\partial R_{\beta \gamma}^{2}}{\partial x_{\beta}}+R_{\beta \beta} \frac{\partial R_{\gamma \gamma}}{\partial x_{\beta}}\right)\right] .
\end{aligned}
$$

As an aside, we note that the Cauchy-Schwarz inequality for $R_{\beta \gamma}$ gives

$$
\left[R_{\beta \gamma}\right]^{2} \leq\left[R_{\beta \beta}\right]\left[R_{\gamma \gamma}\right]
$$

and, in fact for the direct numerical simulations (DNS), $R_{\beta \gamma}=0$ if $\beta \neq \gamma$ and

$$
\frac{\partial D_{\gamma \gamma \beta}}{\partial x_{\beta}}=\frac{\partial}{\partial x_{\beta}}\left[\tau\left(R_{\beta \beta} \frac{\partial R_{\beta \beta}}{\partial x_{\beta}}\right)\right] .
$$

It is interesting to compare these forms to those arising from the model of Daly and Harlow (1970):

$$
\frac{\partial D_{i j k}}{\partial x_{k}}=C_{D} \frac{\partial}{\partial x_{k}}\left[\tau\left(R_{k n} \frac{\partial R_{i j}}{\partial x_{n}}\right)\right] .
$$

For the $D_{\beta \beta \beta}$ component we have

$$
\frac{\partial D_{\beta \beta \beta}}{\partial x_{\beta}}=C_{D} \frac{\partial}{\partial x_{\beta}}\left[\tau\left(R_{\beta \beta} \frac{\partial R_{\beta \beta}}{\partial x_{\beta}}\right)\right],
$$

which is precisely the same form as produced by the Hanjalic and Launder (1972) model for this term. For the $D_{\gamma \gamma \beta}$ component Daly and Harlow (1970) yields

$$
\frac{\partial D_{\gamma \gamma \beta}}{\partial x_{\beta}}=C_{D} \frac{\partial}{\partial x_{\beta}}\left[\tau\left(R_{\beta \beta} \frac{\partial R_{\gamma \gamma}}{\partial x_{\beta}}\right)\right],
$$

which is again precisely the same form as produced by the symmetric Hanjalic and Launder (1972) model for the particular case of our DNS, which represents a "planar" turbulent diffusing layer of initial "quasi-isotropic" turbulence. The primary difference between the two closure approximations is that the symmetric Hanjalic and Launder (1972) model yields a different factor in front of the $D_{\beta \beta \beta}$-term than for the $D_{\gamma \gamma \beta}$-term; Daly and Harlow (1970) does not. Hence the Hanjalic̀ and Launder (1972) form predicts greater 
disparity of diffusion between $R_{\beta \beta \beta}$ and $R_{\gamma \gamma \beta}$ than does Daly and Harlow (1970). One caveat must be noted: If pressure effects are accounted for, one might anticipate that the degree of disparity of diffusion of these two components would probably lie somewhere between that predicted by the Hanjalic̀ and Launder (1972) model and that predicted by Daly and Harlow (1970).

Finally, we present what is perhaps the simplest diffusional form [due to Mellor and Herring (1973)]. Assume that $R_{n k} \approx \delta_{n k} R_{m m} / 3$ and insert this into the Daly and Harlow (1970) model for the term inside the first derivative only to arrive at

$$
\frac{\partial D_{i j k}}{\partial x_{k}}=\frac{C_{D}}{3} \frac{\partial}{\partial x_{n}}\left[\tau\left(R_{m m} \frac{\partial R_{i j}}{\partial x_{n}}\right)\right] .
$$

Clearly, this form predicts exactly the same form of diffusion for both $D_{\beta \beta \beta}$ and $D_{\gamma \gamma \beta}$.

As we have just shown, the so-called gradient diffusion approximation can be thought of as a severe truncation of an algebraic representation of the triple velocity correlations. The initial assumptions leading to the more general algebraic form are similar to the assumptions underlying the Eddy-Damped Quasi-Normal Markovian model of turbulence for homogeneous unforced turbulence. At the severest level of truncation, the analogy to a gradient diffusive process is evidenced by the leading terms in the representation.

Examination of the direct numerical simulation results indicates that the symmetric form offered by Hanjalic and Launder (1972) satisfies the relationships of the tensor components observed in the simulations. Neither the Daly and Harlow (1970) form or the simple form maintain the correct disparity between the diffusion of the various components of the Reynolds stress tensor observed in direct numerical simulations.

\section{E. A Spectral Form for the Triple Correlations}

Attempts to construct a spectral closure for the triple correlations are complicated by (principally) two factors. First, the two-point triple velocity correlations do not satisfy the same symmetry conditions as the single-point form, e.g.,

$$
R_{i j k}\left(\mathbf{x}_{1}, \mathbf{x}_{2}\right)=\overline{u_{i}^{\prime}\left(\mathbf{x}_{1}\right) u_{j}^{\prime}\left(\mathbf{x}_{1}\right) u_{k}^{\prime}\left(\mathbf{x}_{2}\right)} \neq R_{i k j}\left(\mathbf{x}_{1}, \mathbf{x}_{2}\right)
$$

When transformed from $\left(\mathbf{x}_{1}, \mathbf{x}_{2}\right)$ to $(\mathbf{x}, \mathbf{r})$ and Fourier transformed with respect to $\mathbf{r}$, the resulting triple correlation, $T_{i j k}(\mathbf{x k})$, exhibits an asymmetry with regard to its real and imaginary parts. This asymmetry is manifested in the exact k-space spectral transport equation for $E_{i j}(\mathbf{x}, \mathbf{k})$ by the appearance of not only the triple correlations $T_{i j k}(\mathbf{x}, \mathbf{k})$ but also $T_{i j k}(\mathbf{x},-\mathbf{k})$. Thus the simple symmetry arguments used to justify the form of the single-point $R_{i j k}$ does not follow directly in the Fourier space. Second, the relevant time 
scales in the Fourier space for this inhomogeneous process are not obvious. After averaging the equations over angles in $k$-space, one can arbitrarily construct time scales that are either "local" in $k$-space or "nonlocal" (integrated over all wave numbers). BHRZ have offered two models of the triple correlations. The first form, introduced for expedience, does not preserve the tensor properties of either the triple correlations or the triple correlations "augmented" by the pressure-velocity correlations. This first-cut model is simply

$$
D_{i j k}=C_{D} \nu_{t} \frac{\partial E_{i j}}{\partial x_{k}}
$$

where

$$
\nu_{t}=\int_{0}^{\infty} \frac{\sqrt{k E_{n n}}}{k^{2}} d k .
$$

This form was used for the shear-layer computations presented by BHRZ. These computations displayed the emergence of self-similar spectra in which the appropriately scaled spectral shapes for the spectral tensor component varied between components but did not vary across the shear layer. A consequence is that the single-point anisotropy was constant across the entire shear layer. Additionally, the emergence of this self-similarity was highly dependent on the choice of the coefficient $C_{D}$. Turner (1996) has shown that the self-similarity is the result of a delicate balance between the diffusional "velocity" of the $E_{i j}$ given by the simple form (above) and the rate at which the production of $E_{i j}$ moves away from the centerline of the shear layer as $\partial U_{1} / \partial Y$ disperses. The sensitivity to $C_{D}$, the constancy of the anisotropy across the shear layer, and the severe treatment of known symmetries of the diffusion tensor suggest that this simplified model is inadequate. A more general, fully symmetric form was also presented by BHRZ:

$$
D_{i j k}=C_{D} \frac{\partial}{\partial x_{k}}\left\{\eta_{k m} \frac{\partial E_{i j}}{\partial x_{m}}+\eta_{i m} \frac{\partial E_{k j}}{\partial x_{m}}+\eta_{j m} \frac{\partial E_{i k}}{\partial x_{m}}\right\}
$$

where

$$
\eta_{i j}=\int_{0}^{\infty} \frac{E_{i j}}{k \sqrt{k E_{n n}}} d k .
$$

This form alleviates several concerns expressed in regard to the simpler form. First, the emergence of the self-similar form is somewhat less sensitive to the choice of $C_{D}$. Second, the self-similar spectra are consistent with a varying spectral from across the shear layer, and hence, an anisotropy that varies across the shear layer. The principal features of the model predictions are shown in Figs. 2 through 10. Figure 2 shows the evolution of the turbulent kinetic energy $K_{n n}(y, t)$, the turbulent length scale $L(t)=1 / k_{\max }(0, t)$, and 


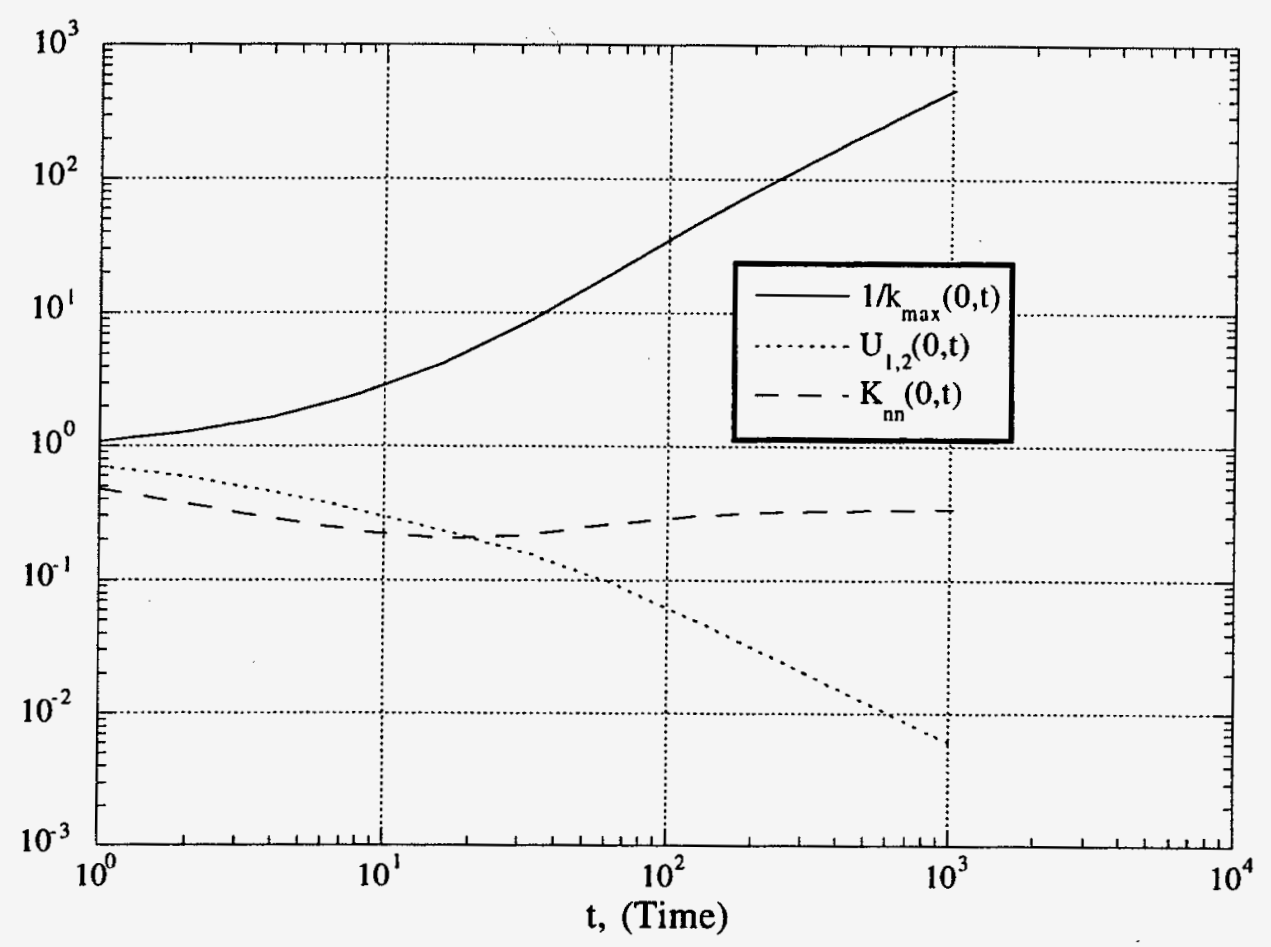

Fig. 2. Temporal evolution of the shear rate, length scale and turbulent kinetic energy along the shear plane at $y=0$.

the shear rate $U_{1,2}(y, t)=\partial U_{1}(y, t) / \partial y$ at the the shear plane, $y=0$. Note that the turbulent length scale at any point in $y, t$ is defined herein as the inverse of the point in $k$-space where the energy spectrum is a maximum, i.e., $k_{\max }(y, t)$. The particular choice of the length scale to scale the data corresponds to the turbulent length scale at the shear plane, $y=0$. This figure show the trend towards the expected self-similar behavior, e.g., constancy of $K_{n n}(0, t)$, linear growth of $L(t)=1 / k_{\max }(0, t)$, and decay of the shear rate inversely proportional to time.

Figure 3 shows the behavior of the normalized turbulent kinetic energy and shear rate as functions of $y * k_{\max }(0, t)=y / L(t)$, the normalized distance from the shear plane. This figure shows a "reasonable" behavior for these quantities. Figure 4 shows the behavior of the turbulent length scale across the shear layer. This figure also shows "reasonable" behavior. It is interesting to note that the length scale of the turbulence does not vanish at the outside edge of the layer. Figure 5 shows the ratio of the length scales of the components of the deviatoric spectral tensor. We may define the deviatoric tensor as

$$
\tilde{E}_{i j}(\mathbf{x}, k, t)=E_{i j}(\mathbf{x}, k, t)-\frac{1}{3} \delta_{i j} E_{n n}(\mathbf{x}, k, t)
$$




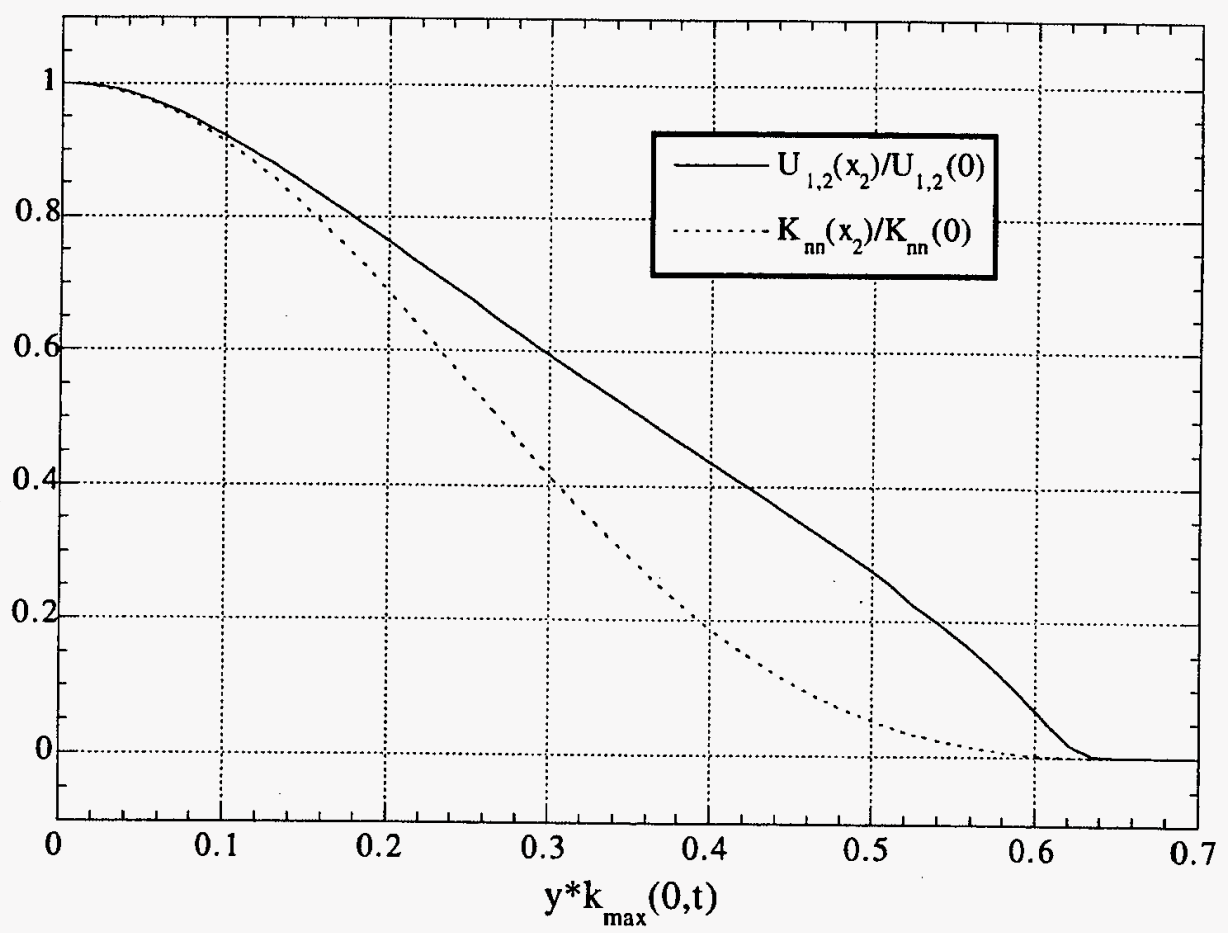

Fig. 3. Variations of turbulent kinetic energy and mean shear rate across the shear layer at $t=1024$.

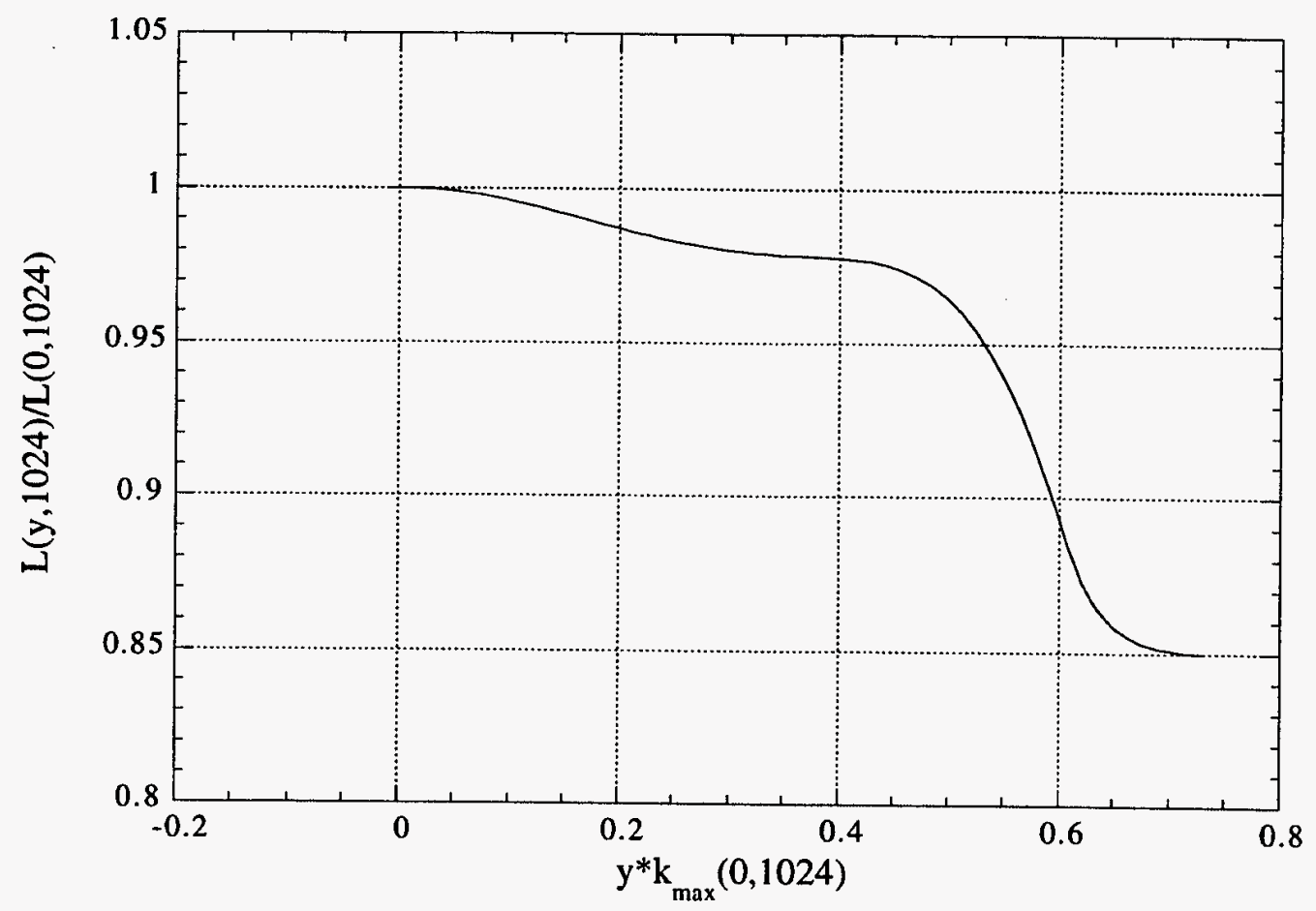

Fig. 4. Variations of turbulent length scale $L(y, t)=1 / k_{\max }(y, t)$ across the shear layer at $t=1024$. 


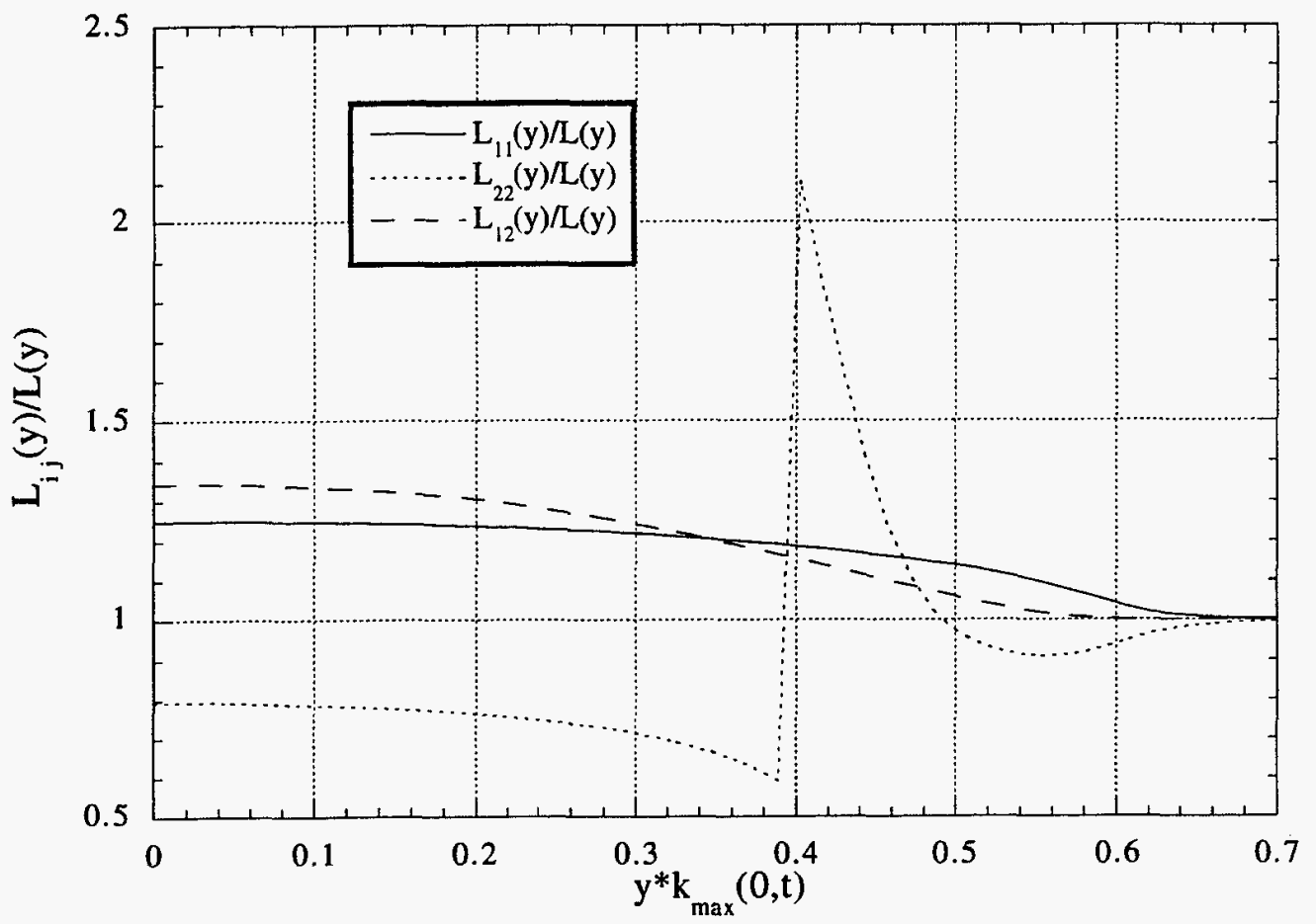

Fig. 5. Variations of the turbulent length scale $L(y, t)=1 / k_{\max }(y, t)$ across the shear layer at $t=1024$.

and denote the wave number at which the $i j^{\text {th }}$ component reaches an extremum as $k_{i j, e x t}(\mathbf{x}, t)$. Then the length scale associated with this component is $L_{i j}(\mathbf{x}, t)=$ $1 / k_{i j, e x t}(\mathbf{x}, t)$. Note that the discontinuity in $L_{22}$ is caused by the change in sign of the $\tilde{E}_{22}$ component. This component changes sign at the lower wave numbers first; when the largest amplitude of the part which has changed sign first exceeds that of the part that hasn't yet changed sign, the corresponding point of the extreme value "jumps" to the extremum associated with the part which has changed sign.

The change in sign of $\tilde{E}_{22}$ is also manifested in the plot of the anisotropy tensor components, shown in Fig. 6. The anisotropy tensor is defined as

$$
b_{i j}(\mathbf{x}, t)=\frac{\tilde{R}_{i j}(\mathbf{x}, t)}{R_{n n}(\mathbf{x}, t)},
$$

where the tilde again denotes the deviator. Note the change in sign of the component $b_{22}$. This figure clearly demonstrates the variation of the anisotropy of the turbulence across the shear layer-a phenomenon missing from computations using the simplest gradient diffusion closures. Note that the same data plotted for a time of $t=512$ produces a graph which is virtually identical to the present graph because the turbulence is essentially selfsimilar in the regime $t>256$. The present result appears to present a reasonable model for 


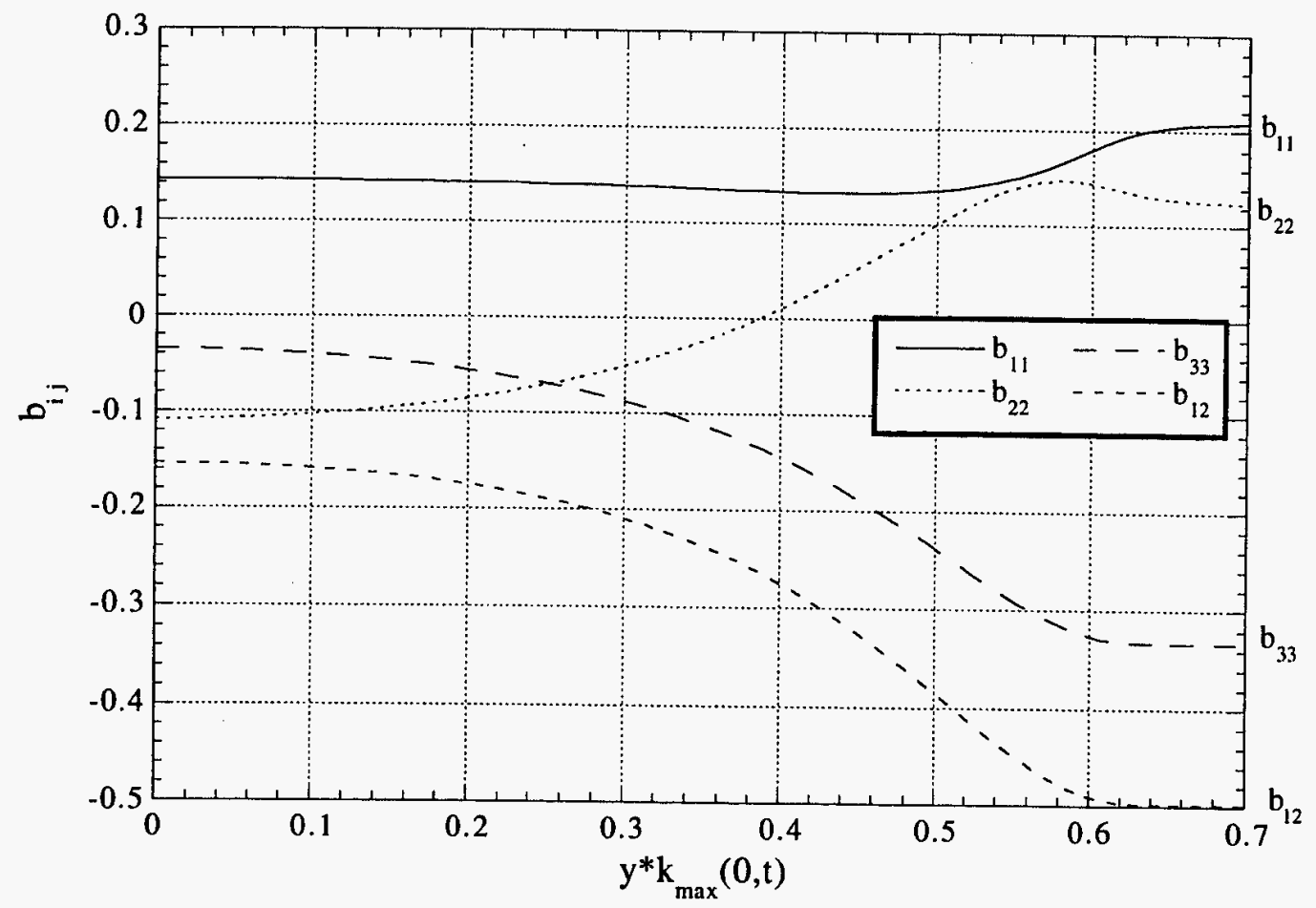

Fig. 6. Variations of components of the anisotropy tensor across the shear layer at $t=1024$.

the variations of anisotropy across the shear layer. The change in sign of the $b_{22}$ component is associated with the predominance of the anisotropic diffusion process (as identified in the DNS) over the "shear production" of negative $b_{22}$ at the outside of the shear layer. Thus, the change in sign of this component is a direct result of the use of the fully symmetric form of the triple velocity correlation closure and gives a result that is consistent with our intuition, which is based on the results of the direct numerical simulations.

The spectral features of the self-similar form are displayed in Figs. 7 through 10. These figures are "shape plots" showing the normalized spectra as functions of the scaled wave number at various values of $y * k_{\max }(0, t)$ and at times of $t=512$ and $t=1024$. Because the turbulence is essentially in the self-similar regime at these times, the data at $t=1024$ overlies the data at $t=512$, for each component at each value of $y * k_{\max }(0, t)$. However, the spectra for a particular component at a specific value of $y * k_{\max }(0, t)$ does not necessarily overlie the values for that component at a different value of $y * k_{\max }(0, t)$. This variation is the spectral manifestation, and indeed the source of the variation of the anisotropy across the shear layer. 


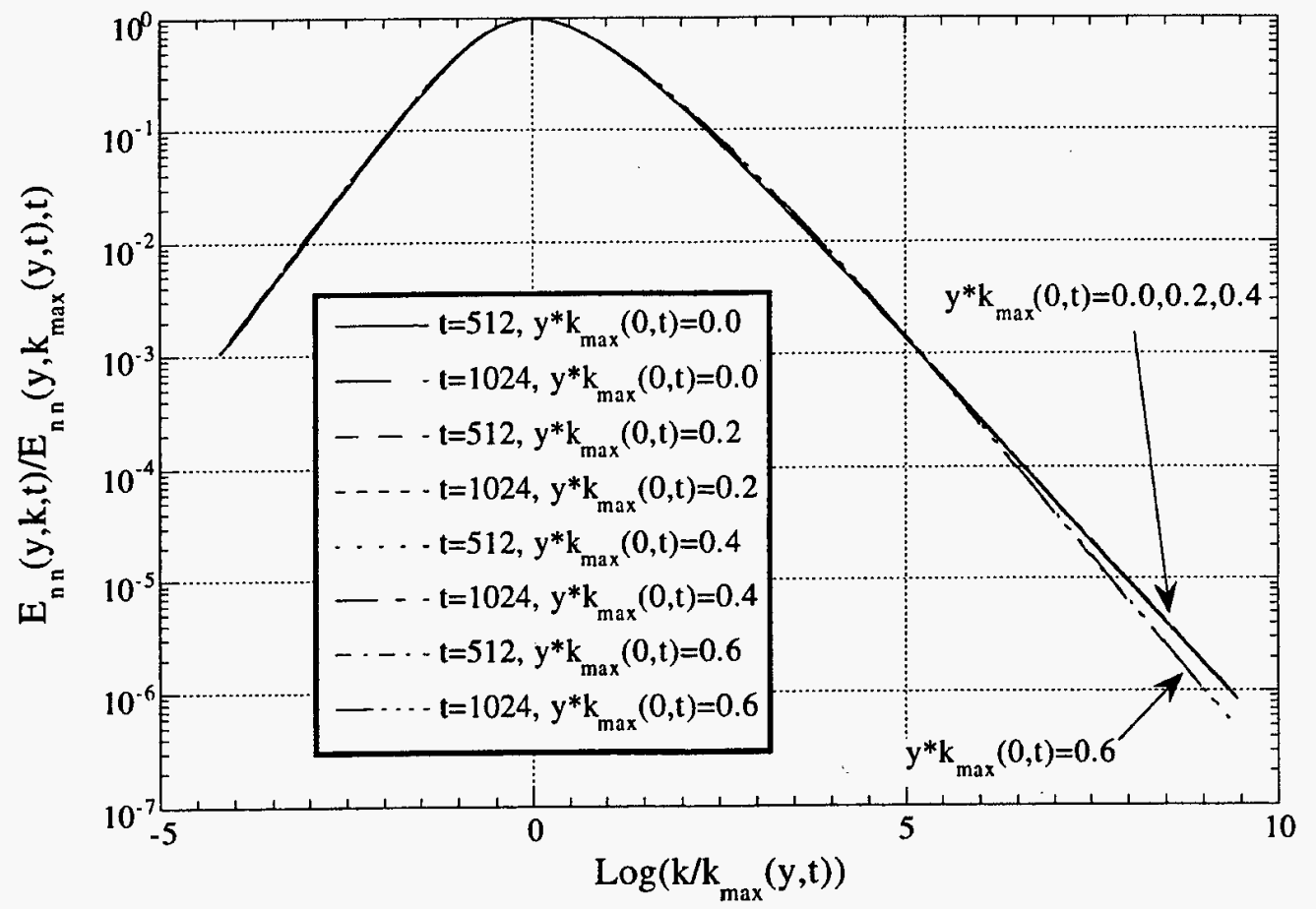

Fig. 7. Variations of the shape of the energy spectrum.

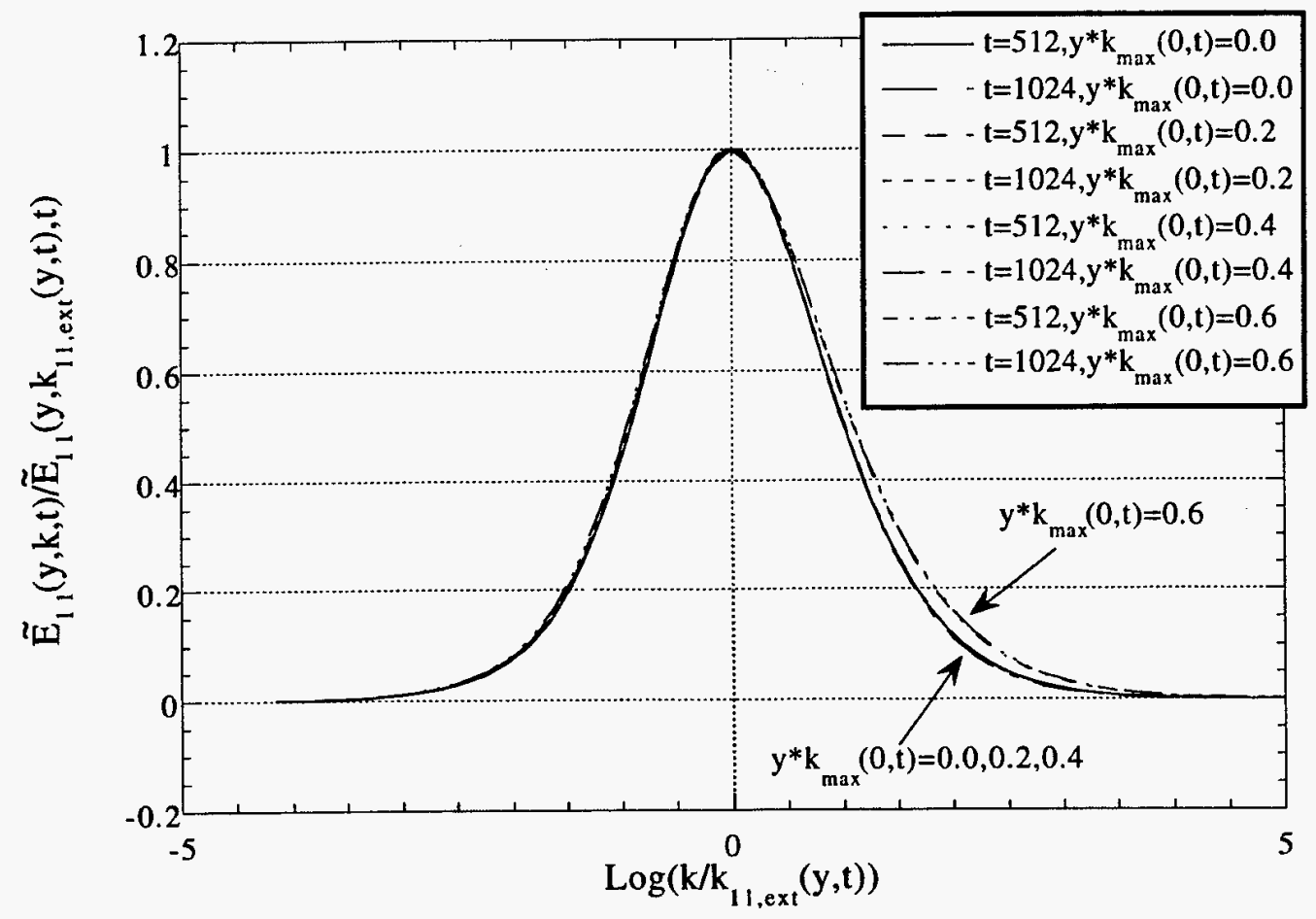

Fig. 8. Variations of the shape of the $\tilde{E}_{11}$. 


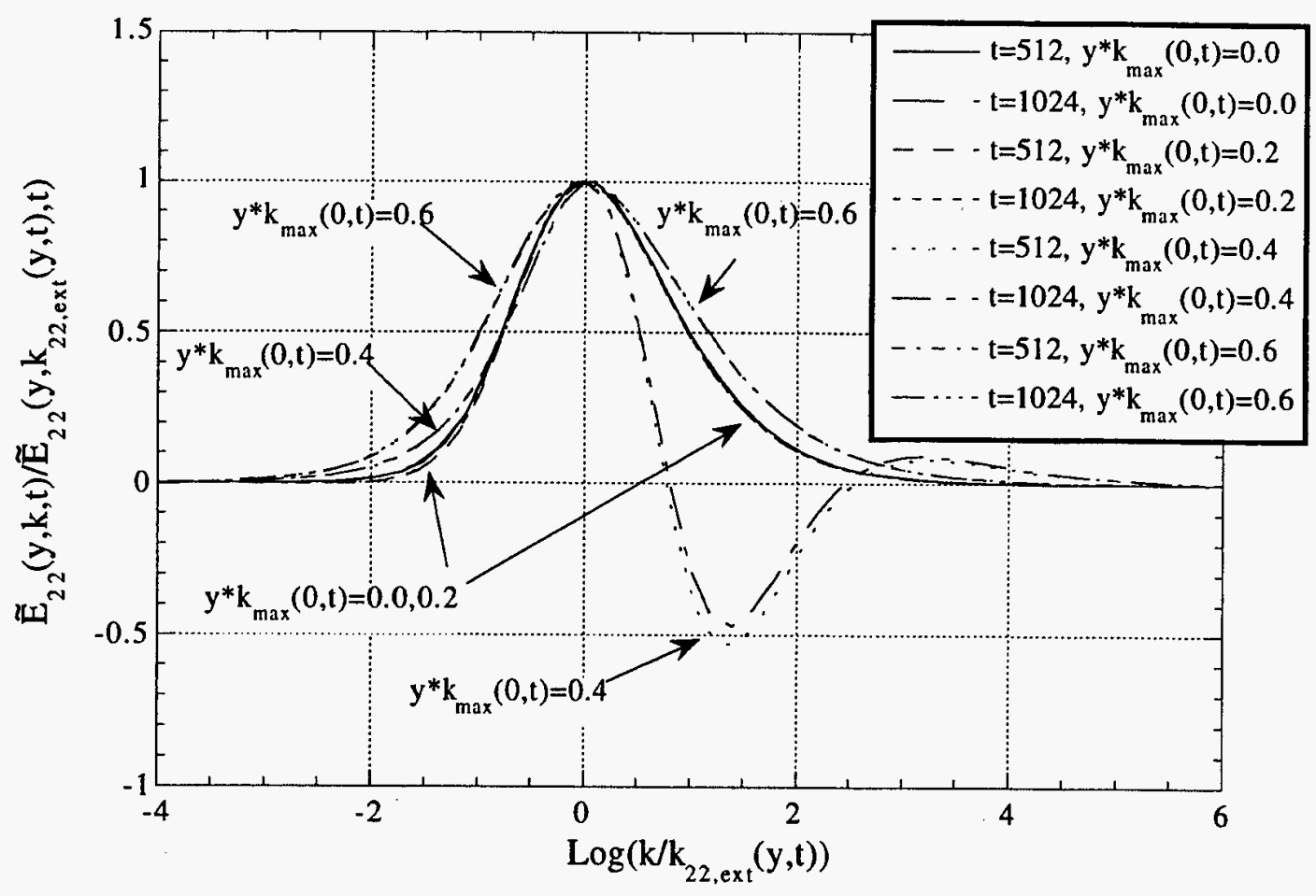

Fig. 9. Variations of the shape of the $\tilde{E}_{22}$ spectrum.

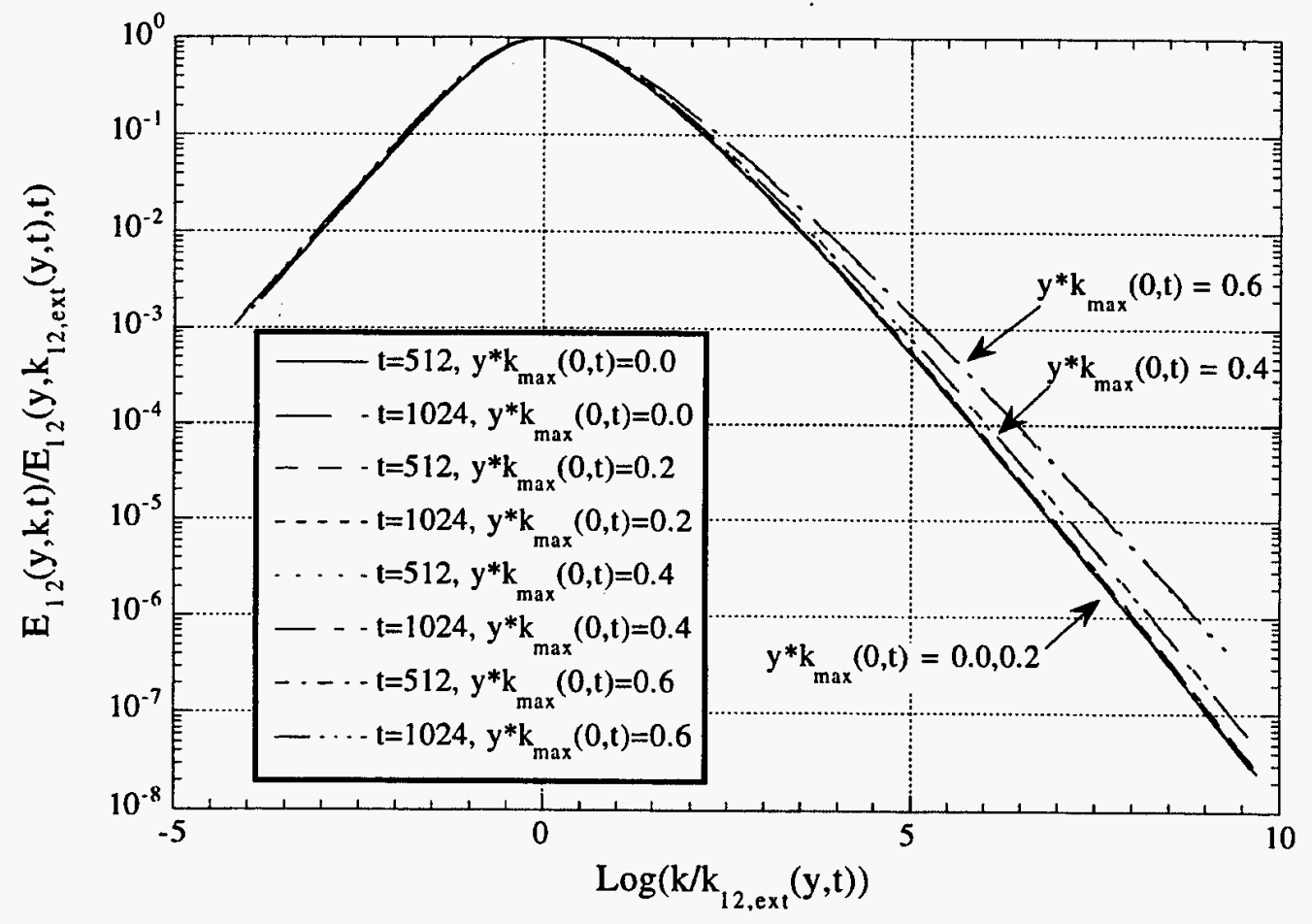

Fig. 10. Variations of the shape of the $E_{12}$ spectrum. 
This symmetric form of the diffusion tensor "mixes" the diffusion of trace and deviatoric parts of the spectral tensor in such a way as to produce a fine-scale "local" anisotropy in the edges of the diffusion layer. This fine-scale anisotropy is most obvious in, say, the $E_{12}$-spectra and might be viewed as a consequence of weak production of turbulence in this vicinity. The production terms balanced against the tendency to return toward isotropy typically produce a $k^{-7 / 3}$ scaling in this model when applied to homogeneous turbulence [Clark (1992)]. However, at the outside edge of this layer, the mixing of the trace components of the spectral tensor with the deviatoric parts imposes a "diffusion" of $k^{-5 / 3}$ behavior onto the deviatoric spectra at the edge. This $k^{-5 / 3}$ behavior is consistent with the idea that the turbulence at the outside of the shear layer is diffusion dominated. It also indicates that the assumption of "local isotropy" may need to be modified for selfdiffusion dominated turbulence. This $k^{-5 / 3}$ behavior may be an appropriate description of the outside edge of the layer, where, in reality, the turbulence is far from Gaussian and far from isotropic, as indicated by the direct numerical simulations. Further studies, both numerical and experimental, would shed much light on this issue.

\section{F. A Spectral Form Including the Pressure-Velocity Correlations}

As noted previously, the effects of the pressure-velocity correlations is to counteract the "diffusive" effects of the triple correlations in a certain preferential fashion. Following the analysis of the previous chapter, this effect can be accounted for in a tensorially correct fashion by defining a new tensor, $\tilde{D}_{i j k}$, such that

$$
\tilde{D}_{i j k}=D_{i j k}-\beta\left(D_{n n i} \delta_{j k}+D_{n n j} \delta_{i k}\right),
$$

where the third-rank tensor, $D_{i j k}$, is fully symmetric on its indices and may be taken as the same symmetric tensor defined above. Note that this "asymmetry" in the indices implies that this tensor appears in the $E_{i j}$ evolution equation with the divergence taken on the $k$-index only. Attempts to use such a closure with the spectral model applied to the free shear layer produced apparently unphysical results. Preliminary examination of the computed results indicate that the inclusion of the pressure effects as a diffusive correction to a gradient diffusion approximation caused pathological behavior near the edge of the

layer. This behavior is apparently the result of the "anti-diffusive" effects of the fluctuating pressure-velocity correlation acting in the direction normal to the shear plane. The physical effect seems to be analogous to a "drag" on the component of the triple correlation that is fully aligned in the direction of the inhomogeneity. (This effect corresponds to the $D_{222}$ component of the diffusion tensor in both the DNS and the model computation.) However, this "drag" effect literally has been modeled as an anti-diffusional correction to 
the diffusive model of the triple correlations. While this form may be adequate for certain components at certain scales, its inclusion in the spectral model applied to the free shear layer apparently caused a spurious "anti-diffusion" for some components at some wave numbers, resulting in the computational failures.

In conclusion, it appears likely that a simple diffusion-like model is inadequate to capture the effects of the pressure-velocity correlations with any real physical fidelity. It seems likely that the nonlocal effects (in physical space) of these correlations are essential to represent the front growth with any real physical fidelity. Much work remains to be done in formulating a physical motivated yet tractable integral (i.e., nonlocal) form for the pressure-velocity correlations. 


\section{References}

Besnard, D., F. Harlow, R. Rauenzahn, and C. Zemach, "Turbulence Transport Equations for Variable-Density Turbulence and Their Relationship to Two-Field Models," Los Alamos National Laboratory report LA-12303-MS (1992).

Besnard, D., F. Harlow, R. Rauenzahn, and C. Zemach, "Spectral Transport Model for Turbulence," Los Alamos National Laboratory report LA-11821-MS (1990); also published in Theoretical and Computational Fluid Dynamics 8, 1-35 (1996).

Clark, T., "Spectral Self-Similarity of Homogeneous Anisotropic Turbulence," Los Alamos National Laboratory report LA-12284-T (April 1992).

Clark, T., and C. Zemach, "A Spectral Model Applied to Homogeneous Turbulence," Phys. Fluids 7, 1674-1694 (1995).

Daly, B., and F. Harlow, "Transport Equations in Turbulence," Phys. Fluids 13, 2634-2649 (1970).

Demuren, A., S. Lele, and P. Durbin, "Role of Pressure Diffusion in Non-Homogeneous Shear Flows," Bull. Am. Phys. Soc. 39, 1954 (1994).

Hanjalic̀, K., and B. Launder, "A Reynolds Stress Model of Turbulence and Its Application to Thin Shear Flows," J. Fluid Mech. 54, 609-638 (1972).

Herring, J., National Center for Atmospheric Research, personal communication, July 1993.

Kraichnan, R., and E. Spiegel, "Model for Energy Transfer in Isotropic Turbulence," Phys. Fluids 5, 583-588 (1962).

Leith, C., "Diffusion Approximation to Inertial Energy Transfer in Isotropic Turbulence," Phys. Fluids 10, 1409-1416 (1967).

Leith, C., "Stochastic Backscatter in a Subgrid-Scale Model: Plane Shear Mixing Layer," Phys. Fluids A 2, 297-299 (1990).

Linn, R. R., "A Transport Model for Prediction of Wildfire Behavior," Ph.D. thesis, New Mexico State University (1997).

Mellor, G., and H. Herring, "A Survey of Mean Turbulent Field Closure Models," AIAA J. 11, 590-599 (1973).

Naot, D., A. Shavit, and M. Wolfshtein, "Two-Point Correlation Model and the Redistribution of Reynold's Stress," Phys. Fluids 16, 738-740 (1973).

Oberlack, M., and N. Peters, "Closure of the Dissipation Tensor Equation and the Pressure Velocity Correlation Based on the Two-Point Correlation Equation," in "Proc. of the 9th Symposium on Turbulent Shear Flows," Kyoto, Japan (1993).

Ohkitani, K., and S. Kishiba, "Nonlocal Nature of Vortex Stretching in an Inviscid Fluid," Phys. Fluids 7, 411-421 (1995). 
Reynolds, W., "Fundamentals of Turbulence for Turbulence Modeling and Simulation," lecture notes for Von Karman Institute, AGARD-R-755 (March 16-17, 1987).

Steinkamp, M., T. T. Clark, and F. H. Harlow, "Stochastic Interpenetration of Fluids," Los Alamos National Laboratory report LA-13016-MS (1995).

Steinkamp, M., "Spectral Analysis of the Turbulent Mixing of Two Fluids," Ph.D. thesis, University of Illinois at Urbana-Champaign (1996).

Steinkamp, M., T. Clark, and F. Harlow, "Two-Point Description of Two-Fluid Turbulent Mixing. Part 1: Model Formulation," Los Alamos National Laboratory document LAUR-96-2038 (submitted to Int. J. of Multiphase Flow).

Steinkamp, M., T. Clark, and F. Harlow, "Two-Point Description of Two-Fluid Turbulent Mixing. Part 2: Numerical Solutions and Comparisons," Los Alamos National Laboratory document LA-UR-96-2039 (submitted to Int. J. of Multiphase Flow).

Turner, L., Los Alamos National Laboratory, personal communication, 1996. 
M98004340

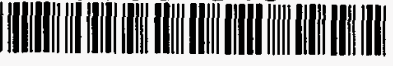

Report Number (14) $L A--1340 /-M S$

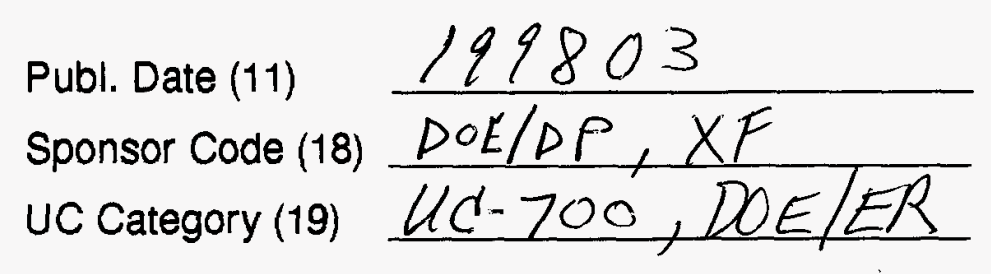

DOE 